\title{
Scaling Theory and Exactly Solved Models In the Kinetics of Irreversible Aggregation
}

\author{
F. Leyvraz \\ Centro de Ciencias Físicas, Universidad Nacional Autónoma de México, Avenida \\ Universidad s/n, Colonia Chamilpa, 62251 Cuernavaca, Morelos, Mexico \\ Contents \\ 1 Introduction \\ 1.1 What is this all about 3 \\ 1.2 Outline of the Paper 8 \\ $\begin{array}{lll}1.3 & \text { Various Topics of Interest not Treated Here } & 10\end{array}$ \\ 2 Irreversible Aggregation: The Mean-field Approach 11 \\ $\begin{array}{lll}2.1 & \text { Generalities } & 11\end{array}$ \\ 2.2 Existence and Uniqueness Results 13 \\ 3 The Scaling Hypothesis 15 \\ 3.1 Basic Concepts: Moments and Typical Size 15 \\ $\begin{array}{lll}3.2 & \text { Different Kinds of Scaling } & 18\end{array}$ \\ 3.3 Some Consequences that Follow from Scaling, and More that Don't 20 \\ 3.4 The Scaling Equation and Its Derivation 23 \\ 3.5 The Solutions of the Scaling Equation 26 \\ $\begin{array}{lll}3.6 & \text { Crossover } & 37\end{array}$ \\ 3.7 Extensions of the Model 40 \\ 3.8 Non-scaling Asymptotics $\quad 52$ \\ 3.9 The Moment Equations $\quad 56$ \\ 4 Exactly Solved Models $\quad 58$ \\ 4.1 The Constant Kernel $\quad 59$ \\ 4.2 The Sum Kernel 62 \\ 4.3 Parity-dependent Kernels 66 \\ $\begin{array}{lll}4.4 & \text { The } q \text {-sum kernel } & 70\end{array}$ \\ 4.5 The Bilinear Kernel 73 \\ $\begin{array}{ll}\text { 4.6 Crossovers in the Bilinear Kernel } & 77\end{array}$ \\ $\begin{array}{llr}4.7 & \text { Finite Systems } & 80\end{array}$ \\ 4.8 Pure Three-body Reactions $\quad 85$ \\ 4.9 Three-body Reactions Perturbing Two-body Aggregation 88
}

Preprint submitted to Elsevier Science 29 October 2018 
4.10 Constant Kernel with Production Term 90

$\begin{array}{ll}4.11 \text { The constant kernel with a localized source term } & 92\end{array}$

$5 \quad$ Beyond Mean-field $\quad 93$

5.1 Diffusion-limited Cluster-Cluster Aggregation 94

$\begin{array}{lll}5.2 & \text { Ballistic Aggregation } & 102\end{array}$

$\begin{array}{lll}6 & \text { Conclusions and Outlook } & 110\end{array}$

$\begin{array}{ll}\text { Acknowledgements } & 112\end{array}$

A Derivation of the Scaling Equation for $\Phi(x) \quad 112$

B Derivation of the Scaling Equation in the Three-Body Case 114

$\begin{array}{lll}\text { C The Large- } \rho \text { Behaviour of } I(\rho) & 115\end{array}$

D The Large- $\rho$ Behaviour for the Three-body Reaction 117

E The Distribution of Compositions in Multicomponent Aggregation 118

F The Integral Equation for the Gelling Case 119

G The Sum Kernel: The Technique of Characteristics 122

H The Parity Dependent Kernel: Scaling Theory 124

$\begin{array}{ll}\text { I The } q \text {-sum Solution: A Sketch } & 128\end{array}$

$\begin{array}{ll}\mathrm{J} & \mathrm{Crossover} \text { in the } q \text {-sum kernel } \\ \end{array}$

K The Simple Product Kernel: Technicalities 131

L The Product Kernel: Power-law initial Conditions 135

M The Full Bilinear Kernel: A Sketch of the Scaling Theory 137

N The $B \rightarrow 0$ Crossover When $C=0 \quad 139$

O The $C \rightarrow 0$ Crossover When $B=0 \quad 141$

P The Solution of the Finite Constant Kernel 142

$\begin{array}{ll}\text { Q The Finite-sum Kernel } & 144\end{array}$

$\begin{array}{lll}\text { R The Three-body Kernel } m_{1}+m_{2}+m_{3} & 146\end{array}$

S The Kernel $m_{1}+m_{2}+m_{3}$ Perturbing the Constant Kernel 148

$\mathrm{T}$ The Scaling Function for the One-dimensional PCM 151

U Ballistic Aggregation: Sketch of Exact Solution 152

$\begin{array}{ll}\text { References } & 153\end{array}$

\begin{abstract}
The scaling theory of irreversible aggregation is discussed in some detail. First, we review the general theory in the simplest case of binary reactions. We then extend consideration to ternary reactions, multispecies aggregation, inhomogeneous situations with arbitrary size dependent diffusion constants as well as arbitrary production terms. A precise formulation of the scaling hypothesis is given as well
\end{abstract}


as a general theory of crossover phenomena. The consequences of this definition are described at length. The specific issues arising in the case in which an infinite cluster forms at finite times (the so-called gelling case) are discussed, in order to address discrepancies between theory and recent numerical work. Finally, a large number of exactly solved models are reviewed extensively with a view to pointing out precisely in which sense the scaling hypothesis holds in these various models. It is shown that the specific definition given here will give good results for almost all cases. On the other hand, we show that it is usually possible to find counterexamples to stronger formulations of the scaling hypothesis.

Key words: aggregation, scaling, exactly solved models PACS: 05.70.Ln, 82.70, 82.35.+t

\section{Introduction}

\subsection{What is this all about}

What do a glass of fresh milk, a planetary system in formation and Los Angeles on a smoggy day all have in common? At first sight, not much, even from the slightly skewed viewpoint of a physicist. The various phenomena involved in these systems have quite different physics and the interesting questions are wholly unrelated. Nevertheless, there is one aspect which these three (and a large number of other) systems share: in all of them one finds some process of irreversible aggregation, the characterization of which is of some importance. To be specific, in the case of milk, we are dealing with small globules driven by buoyancy which coalesce irreversibly to form cream; in a planetary system, on the other hand, one has planetesimals colliding inelastically to form ever larger blocks, eventually leading to planet formation. Finally, in aeorosols such as those found in smog, we have airborne particles so small that Brownian motion is (frequently) an important transport mechanism. There the aggregation mechanism is due to the presence of van der Waals forces between the particles, which are considerably stronger than the effects of thermal agitation.

Let us therefore review what these systems have in common: in all of them, we have identifiable aggregates of particles playing an important role. These aggregates grow by sticking to each other. Specifically, they are usually not limited to growing by accretion of the very smallest clusters, which is another topic altogether. One has, therefore, a situation in which there is originally quite a large number of small particles, and these then coalesce as time goes

Email address: leyvraz@fis.unam.mx (F. Leyvraz). 
on. No allowance is made for the possibility of break-up. One is thus not concerned with a problem of approach to equilibrium, but with an entirely dynamical problem ${ }^{1}$.

Aggregation phenomena are, as hinted at above, quite common in nature. Apart from the examples mentioned above, we may mention astrophysics (for further discussion of the subject with references see e.g. [55]), cloud physics (see [86]) and polymer chemistry, where a great deal of the theoretical work underpinning the gelation transition actually originated (see [95]). Even to attempt to give a fair idea of the variety of applications of aggregation and related concepts, however, is beyond both my abilities and the scope of this work. The reader specifically interested in the applications to aerosol physics is referred to the review by Drake [23] which addresses a large number of such issues in detail. Friedlander [32] and Hidy [37] are also important general references on the subject.

What, then, are the issues we want to address when dealing with such a system? Overall, the questions most frequently asked fall into two categories: the first concerns the sizes and the second the morphology of the aggregates. Let us deal shortly with the latter first. It is, of course, quite obvious that if two liquid droplets coalesce, they will, under the influence of surface tension, rapidly relax to a spherical shape. Slightly more complex liquid systems may behave in a less obvious way (think for example of a water drop on an inclined glass plane, where surface tension and gravity act together), but the geometry still remains fairly straightforward. Matters change dramatically, however, when solid particles stick together. In this case, depending on the degree of rigidity of the bonds, the aggregates may or may not rearrange. The simplest case, because it is quite well-defined, is the one in which there is no rearrangement whatever. Then, as is well-known, the aggregates grow fractal shapes and their morphology can only be characterized at the statistical level. The main tool used in order to characterize different morphologies is the so-called mass-radius relationship

$$
R(m)=\text { const. } \cdot m^{1 / D_{f}},
$$

for large values of the mass $m$. Here $R(m)$ is some average measure of the radius of the aggregate as a function of its mass $m$ and $D_{f}$ is an exponent known as the fractal dimension. A considerable amount of work has gone into the study of such systems (for a review and for an introduction to the extensive literature, see $[47,78])$. The upshot is that a fractal dimension can indeed be defined, but that it depends, although not very sensitively, on some of the

1 It is argued by some that this is unrealistic, since on fundamental grounds backward reactions always exist. To this the short answer is that I shall only be interested in time scales for which the backward processes can indeed be neglected. 
details of the transport mechanism. In particular, ballistic transport yields more compact structures (and hence larger values of the fractal dimension $D_{f}$ ) than diffusion, and low sticking probability has a similar effect.

A short summary of the above discussion could therefore be stated as follows: morphology is extremely important, but it is strongly dependent on the specific mechanisms involved in each particular system (many features of general interest such as, say, the appearance of complex self-similar structures in the case where no restructuration is present, occur quite generally, but detailed features, such as the actual values of the fractal dimension, do depend on specifics).

What will principally concern us in this review is the study of the masses of the aggregates. Specifically, since there are always a large variety of masses at one given time, we need the so-called cluster size distribution as a function of time. (Note that in the following, by size, we shall always mean mass and never some measure of geometric size such as radius or volume.) By this we mean that we study the function $c(m, t)$ giving the concentration of aggregates of mass $m$ at time $t$. Roughly speaking, one uses the following strategy: in many physical situations, it is possible to express the rate of aggregation as the encounter probability between aggregates of masses $m$ and $m^{\prime}$, multiplied by a reaction rate $K\left(m, m^{\prime}\right)$ depending in quite a general way on the two masses $m$ and $m^{\prime}$. The possibility of describing the aggregation process in this very simplified manner depends on several assumptions, which will be shortly discussed later. The most important is a mean-field hypothesis, which states that no spatial correlations develop during the course of the reaction. From the theoretical point of view, this is an extremely severe assumption, but in practice it is often quite well satisfied.

If this can be done, it means that we have summarized all the information concerning the complicated and specific physics of the various individual systems in the matrix $K\left(m, m^{\prime}\right)$ characterizing the mass-dependence of the reaction rates. It should be emphasized that the determination of the rates $K\left(m, m^{\prime}\right)$ is in general by no means straightforward. However, as it depends intimately on the specific physics of the system involved, we shall not consider it here, but rather always assume the rates $K\left(m, m^{\prime}\right)$ to be externally given. One is then led to a system of infinitely many coupled non-linear rate equations. These still present a formidable problem, which usually cannot be solved by analytic means. As for numerical studies, while they are certainly very valuable, they are necessarily limited in their ability to deal with either large cluster sizes or large times. In this review, we extensively describe an approach due originally to Friedlander, and then considerably expanded upon by Ernst and van Dongen, namely the scaling approach. The crucial idea consists precisely in limiting oneself to the case of large masses and large times. One may then, as was shown, take only the crudest features of $K\left(m, m^{\prime}\right)$ into account and 
arrive at quite precise predictions for the overall features of the cluster size distribution.

Let us illustrate this by a simple and classical example: consider particles moving with a mass-dependent diffusion constant $D(m)$ having a radius $R(m)$ growing as in (1.1). It is then reasonable to assume that the reaction rates are given by

$$
K\left(m, m^{\prime}\right)=\left(D(m)+D\left(m^{\prime}\right)\right)\left(R(m)+R\left(m^{\prime}\right)\right)^{d-2},
$$

where $d$ is the dimension of space. This follows from the elementary fact that the rate at which a pointlike particle diffusing with diffusion constant $D$ is captured by a spherical trap of radius $R$ is of the order $D R^{d-2}$. In order to proceed, we need to know the asymptotic behaviour of the diffusion constants $D(m)$. Let us assume, in keeping with tradition, a fully general dependence of the type

$$
D(m)=\text { const. } \cdot m^{-\gamma} \text {. }
$$

The scaling theory referred to above then states the following facts concerning the cluster size distribution $c(m, t)$, that is, the number of aaggregates of mass $m$ per unit volume present in the system at time $t$ :

(1) The so-called typical cluster size $s(t)$ grows as $t^{z}$, where the exponent $z$ is given by

$$
z=\frac{D_{f}}{D_{f}+\gamma-(d-2)}
$$

This means, as we shall see in far greater detail. that if we consider an appropriate rescaling of the cluster size distribution on this scale, it collapses onto a single time-independent distribution.

(2) From the above follows also that

$$
M_{p}(t):=\int_{0}^{\infty} m^{p} c(m, t) d m=\text { const. } \cdot t^{(p-1) z},
$$

at least for sufficiently large values of $p$. Further work shows that if $\gamma>0$, which is certainly the most common case, (1.5) actually holds for all values of $p$. When $\gamma \leq 0$, on the other hand, the critical value for $p$ is not known, but is known to lie between 0 and $(d-2) / D_{f}-\gamma$.

(3) Finally, the behaviour of the aggregates that are much smaller than $s(t)$ can be evaluated. Thus it can be shown that such aggregates decay as $\exp \left(-\right.$ const. $\left.\cdot t^{(d-2) / D_{f}}\right)$ if $\gamma<0$, whereas they decay as a power law otherwise. 
The way in which these and similar conclusions are drawn will be the topic of this paper.

At this stage, the better read may be experiencing some doubts: all of the above example, in fact, was essentially known in the sixties, see for example [96]. So why am I talking about such things, and what is new? The answer comes in two parts.

First, I have attempted to show how scaling theories can be constructed in a nearly automatic manner for almost any type of problem involving irreversible aggregation. By this I mean that I have treated a large number of extensions (source terms, aggregates with variable compositions, inhomogeneous situations and, finally, the effect of three-body interactions). In all of these cases I have shown a perfectly routine way of obtaining a scaling theory, which yields sensible results. The point of this excercise, which may be said to be the main purpose of this review altogether, is to show how to compute scaling functions systematically in almost any reasonable situation by simply turning a crank. In this respect, the case of three-body interactions is particularly instructive: indeed, I was just busy working out just how the crank worked in this particular case, when suddenly it failed in quite an unexpected way. What happened is that an exact result, quite easy to derive, conflicted with the equally evident scaling theory I had devised. Appendix B presents my present view of what went wrong, and should be borne in mind whenever constructing any kind of scaling theory along these lines. Another rather tricky issue concerns gelation: in this case, all the original literature had predicted certain values for various exponents, which were clearly refuted in the remarkable numerical simulations of Lee [56]. An attempt is made to show that the previous arguments $[59,116,117]$ were indeed inconclusive ${ }^{2}$, to show in detail why and to present some indication of how a theory of Lee's work might go.

As the title of this work indicates, however, there is yet another side to this review, which concerns exactly solved models. Indeed, there exists a considerable body of knowledge concerning those few models for which the rate equations can be solved analytically. This is extremely useful in order to test the scaling approach. The problem is that the exact nature of the error made when using the scaling ansatz is not known a priori. On the other hand, when one is dealing with exactly solved models, one has a full overview of the solution's behaviour, so that a detailed comparison with the scaling predictions becomes possible. To this end, however, it is necessary to investigate the exact solutions in the case of arbitrary initial conditions, which has not, to my knowledge, been done earlier to any great extent. Here I present such exact solutions and show that scaling, in a very specific sense of the word, indeed invariably holds, whereas many of the consequences which are ordinarily be-

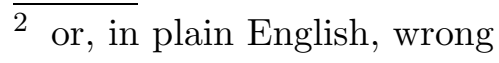


lieved to follow directly from it, actually fail. This may be viewed as a list of cautionary counterexamples, which show the need for care in stating what follows from scaling and what does not (thus, I have been characteristically careless in asserting, for example, the behaviour of the small clusters stated in the example above). The whole issue is rather complex, but a simple summary ${ }^{3}$ may be given as follows: whenever a given property of the cluster size distribution involves a finite fraction of the total mass, one may be reasonably confident to obtain it correctly via scaling. Whenever it does not, however, counterexamples are possible, and indeed, in generic cases, rather likely.

\subsection{Outline of the Paper}

Let me here briefly summarize the layout of this paper. In Section 2 I state the known results on the properties of existence, uniqueness and mass conservation for the solutions of the rate equations. These are rather few, but quite instructive. Indeed, they are probably also in some sense optimal, according to the results suggested by the scaling approach. In Section 3 we develop the scaling approach in several stages. First, we show how scale invariant solutions exist whenever the rate kernel $K\left(m, m^{\prime}\right)$ is homogeneous in its arguments. This in itself, however, says nothing about the general solutions of the equations. To this end, we require the scaling hypothesis, which states that all solutions which start from an specific class of initial conditions eventually approach this scale invariant solution at large times. We then go on to discuss the precise sense in which such convergence should be assumed. This question may seem to be entirely technical and devoid of interest, but in fact it lies at the very heart of the issues concerning the validity of scaling. I shall suggest that the appropriate choice of mode of convergence is weak convergence, where suitably normalized measures are defined in terms of the cluster size distribution $c(m, t)$. To express this in non-technical terms, I will say that a cluster size distribution $c(m, t)$ converges weakly to a given distribution $\Phi(x)$ with respect to a typical size $s(t)$ if the expectation value of any function which varies smoothly on the scale $s(t)$ tends to the corresponding expectation value as $t \rightarrow \infty$. If this specific type of convergence is used, it can be shown, as will be done extensively in Section 4, that scaling obtains under all reasonable circumstances. In other words, all the issues that have been raised concerning so-called "violations of scaling" are seen to arise from the fact that the correct questions are not being asked, or equivalently, that conclusions are being drawn from the scaling hypothesis, which are not legitimate once scaling is defined in this precise manner $^{4}$. I then proceed to derive a general integral equation for the

3 valid at least for the non-gelling case

4 Before scaling is defined in some way or other, of course, the whole debate remains void 
scaling function $\Phi(x)$. This is simply a mild generalization of the Ernst-van Dongen equation and allows in the usual way to derive the standard cases I, II and III ${ }^{5}$ for non-gelling aggregation. A similar equation is also obtained for the gelling case, and some very partial results for the behaviour of the solution at the origin are obtained. These, while still quite incomplete, clearly show that the original claims of [58] concerning the so-called $\tau$-exponent in the gelling case were incorrect. The resulting claims are now more nearly in agreement with the numerical findings of Lee [56]. The theory further suggests a new scaling relation which should, perhaps, be verified numerically. After these general remarks, I proceed to show how the scaling approach can always be obtained in an essentially mechanical fashion. In particular, extensions to monomer production, spatially inhomogeneous systems, aggregation with aggregates of many species multicomponent aggregation and, finally, three-body aggregation, are all worked out in some detail. A theory of crossover, for the case in which one aggregation mechanism is supplanted by another at large sizes, is also presented. In Section 4, on the other hand, we run through most of what was worked out in Section 3, but now using exactly solved models. Our main workhorse, of course, is the constant kernel, for which a great deal is known, and which can be solved exactly in almost any of the extensions one cares to think about. However, we also consider the sum and product kernels, and present some results on the general bilinear kernel, in order to illustrate the concepts of crossover previously developed. We also discuss two more recent exactly solved models, both of them variations on the constant kernel: one is a set of reaction rates $K(k, l)$ which depend on the parity of the sizes $k$ and $l$ of the reactants, but on nothing else; the other is also only defined for discrete values of the masses and is given by $2-q^{k}-q^{l}$, where $q$ is an arbitrary number between zero and one. Both of these can be solved exactly to a large extent, and both display strange effects which have occasionally been argued to be "violations of scaling". Again, a discussion of these models' behaviour shows the contrary, but also sheds considerable light on the limits of our definition of scaling, which has a very hard time making any reasonable statements about clusters of fixed size. But then, as these models in fact show, the behaviour of fixed size clusters can be completely unexpected. Finally, in section 5, I discuss the issue of models for which the mean-field theory does not apply. In the cases I discuss, spatial correlations are built up by the interplay between transport and reaction. Whenever the reaction is slow, these are negligible, but as soon as transport becomes the rate-limiting factor, spatial correlations invalidate the use of the rate equations. In this case, no straightforward application of the formalism developed in the previous sections is, of course, possible. Scaling concepts, however, remain extremely useful as we shall see, as they allow to analyze the information one has in a systematic way.

$\overline{5}$ These will be described in due time. 


\subsection{Various Topics of Interest not Treated Here}

There are, as always, a considerable number of interesting subjects which could, and perhaps ought to, have been included in this review, but which have been left out, for reasons either of space, of limited competence on my part, or else finally because, although undeniably beautiful, certain subjects do not fall readily within the scope of this paper as it has been described in the previous subsections. A necessarily incomplete list might include the following

(1) The rate equations are not, of course, primary: rather they are derived, after some approximations, from a master equation for some appropriate Markov process. Using then the so-called $\Omega$-expansion devised by van Kampen, one may derive the rate equations. This leads to the discussion of corrections due to particle number fluctuations, for a thorough discussion of which the reader is referred to $[70,104,105,109,11]$. A considerable literature has also arisen in the literature on probability concerning such models and their connection to the Smolchowski equations. For a review, see [2]. An altogether remarkable claim of convergence of the above stochastic process to the rate equations treated in this paper is made in [81].

(2) It is possible to go into much greater detail concerning the exact solution of the full bilinear kernel. I have essentially limited myself to analyzing various types of crossover and showing that scaling behaviour arises for arbitrary initial conditions. Much more explicit forms can in fact be obtained, and a very interesting combinatorial interpretation of them can be given. The reader is referred to $[90,91,92]$ for further discussion of these issues.

(3) The list of extensions to the aggregation equations is of necessity incomplete. Thus one findstreatments of aggregation with replication or with annihilation $[52,6]$ as well as much more. The elements presented in this paper could also be multiplied in various ways. This has not been attempted. Rather, I have tried to emphasize the applicability of a general method, which could then be carried over to arbitrary cases as desired. The systems studied were chosen on the basis of a wholly subjective feeling concerning both their practical importance and the relevance of the conceptual issues attached to them.

(4) As will be seen, there exist several cases in which the polydispersity exponent $\tau$ cannot be determined via a straightforward study of the integral equation for the scaling function. For these a very powerful method was developed by Cueille and Sire [18] in order to obtain rigorous bounds on the $\tau$ exponent. The method turns out to be so powerful that these bounds are often tantamount to exact evaluations: the upper and the lower bounds coincide to, say, four or five decimals. They also give a systematic approach to make ever better bounds. This should therefore be 
viewed as a solution of the problem of evaluating $\tau$ for those particular cases. However, the approach is quite subtle, so that I must refer the reader to the original paper.

(5) My treatment of non mean-field models is completely insufficient. This, however, may perhaps be excused in the light of the fact that my aim here is to present the scaling theory of aggregation, not all the beautiful results in some way connected with irreversible processes and models for chemical reactions, such a task being quite beyond my abilities. I have therefore strictly limited myself to such models as were strictly aggregation models, and have only shown how the scaling theory can be brought to bear in such case. Even so, I have not, of course, been able to do the subject justice. The references given in this Section may get the reader started.

\section{Irreversible Aggregation: The Mean-field Approach}

\subsection{Generalities}

As already stated in the Introduction, the phenomenon of irreversible aggregation is described as follows: Aggregates of mass $m$ and $m^{\prime}$ react to form aggregates of mass $m+m^{\prime}$. In mean-field theory, one writes down rate equations for this process, asssuming that

$$
A_{m}+A_{m^{\prime}} \underset{K\left(m, m^{\prime}\right)}{\longrightarrow} A_{m+m^{\prime}}
$$

where the $K\left(m, m^{\prime}\right)$ are the rates at which the aggregation process takes place. The index $m$ may range over a discrete or a continuous range of values. If we then denote by $c(m, t)$ the concentration of aggregates $A_{m}$ at time $t$, we obtain the following rate equations to describe the dynamics of the cluster size distribution

$$
\begin{aligned}
\partial_{t} c(m, t)= & \frac{1}{2} \int d m_{1} d m_{2} K\left(m_{1}, m_{2}\right) c\left(m_{1}, t\right) c\left(m_{2}, t\right) \times \\
& \times\left[\delta\left(m_{1}+m_{2}-m\right)-\delta\left(m_{1}-m\right)-\delta\left(m_{2}-m\right)\right] .
\end{aligned}
$$

Here, as in the rest of this paper, I choose the convention that $m$ runs over continuous values, since the modifications to obtain the discrete case can always be viewed as specializations to singular measures. However, since in many cases it is much more convenient to solve the case in which only discrete multiples of a certain mass occur, let me introduce some notations which will recur throughout this paper: if we have discrete initial conditions, that is, if there 
is an $m_{0}$ such that

$$
c(m, 0)=\sum_{k=1}^{\infty} c_{k}(0) \delta\left(m-k m_{0}\right)
$$

then the solution $c(m, t)$ at all times has the form

$$
c(m, t)=\sum_{k=1}^{\infty} c_{k}(t) \delta\left(m-k m_{0}\right) .
$$

Here the r.h.s of (2.4) defines the functions $c_{k}(t)$. A very important special case is that of so-called monodisperse initial conditions, for which $c_{k}(0)=\delta_{k, 1}$.

The derivation of (2.2) involves certain hypotheses, which I shortly discuss: First, the effect of cluster morphology on the rates of aggregation must either be neglected or be taken into account using some appropriate averaging techniques. Second, we need to assume that no spatial correlations between the clusters build up as the reaction proceeds. It is well-known that such an assumption is by no means trivial. In fact, one may argue that most realistic systems will, for sufficiently large times, display such correlations. However, it can be shown that these can indeed be neglected when the transport mechanisms responsible for bringing the clusters to react are significantly faster than the reaction step. Since this is a fairly common situation in practice, we will restrict ourselves to this mean-field situation except in Section 5.

Equations (2.2) can, of course, be generalized in many ways. One may include, among others, the following additional effects:

(1) Reactions of higher order than binary. This is certainly important when the volume fraction of the aggregates grows in time, which occurs whenever a model displays fractal growth. In this situation, however, it is quite unlikely that the hypotheses necessary for mean-field theory to hold remain valid when these terms become dominant. However, their effect as corrections can be estimated within this framework, which allows to decide whether such corrections are likely to be important or not.

(2) The parameter $m$ can be generalized to be a multicomponent vector. This describes, for example, situations in which the aggregates consist of various substances. In this case the scaling theory must be generalized somewhat to incorporate various different mass scales which describe different aspects of the cluster size distribution, namely the average as well as the spread in composition, which both scale differently, due to the central limit theorem.

(3) Spatially inhomogeneous situations can be considered. In this case, diffusion terms must be explicitly added to the equation. The diffusion con- 
stants involved have, of course, in general a non-trivial mass-dependence, which leads to quite complicated equations.

(4) Finally, monomer production can be introduced. One may either consider the case in which this occurs homogeneously throughout the system, or on the contrary, we may assume that monomer is being produced at a given point and diffuses as it aggregates.

As we shall see, the scaling approach can be extended to give a straightforward description of these various systems. However, before we proceed, I would like to review shortly some of the rigorous results known on the existence and uniqueness properties of solutions of (2.2).

\subsection{Existence and Uniqueness Results}

In the following, we shall treat the discrete case of (2.2) first: results have usually first been obtained for it, since the general case involves some additional difficulties. These equations represent an extremely challenging problem from the point of view of pure mathematics. The reason is that they consist of an infinite number of coupled nonlinear ordinary differential equations. There do not exist standard results for such cases.

In this case, existing results fall into two classes: First, those which make an assumption of the type:

$$
K\left(m, m^{\prime}\right) \leq C\left(m+m^{\prime}\right)
$$

Here, and throughout the rest of this paper, reaction rates will always tacitly be assumed to be positive. From such an assumption it can be shown [112] that the $c(m, t)$ decay exponentially in $m$ for all $t$ for which the solution exists. From this one shows that the quantity

$$
M_{1}(t)=\sum_{m=1}^{\infty} m c(m, t)
$$

remains constant as long as the solution is defined. This in turn allows to prove existence and uniqueness of the solution for all times. Note that it is formally trivial to prove the constancy of $M_{1}(t)$, which physically corresponds to the conservation of the total mass contained within the aggregating system. However, from a rigorous point of view, it is necessary that

$$
\int_{0}^{\infty} m K\left(m, m^{\prime}\right) c(m, t) c\left(m^{\prime}, t\right) d m d m^{\prime}<\infty
$$


for all times.

These results are in a sense optimal: they yield both existence and uniqueness, as well as mass conservation. Further, it is expected that mass-conservation will fail whenever the hypothesis (2.5) does. However, this hypothesis is often too restrictive. Thus, a reasonable model for aggregation of branched aggregates (without loops) assumes that the number of reactive sites on each aggregate grows as $m$, from which follows

$$
K\left(m, m^{\prime}\right)=C m m^{\prime}
$$

which obviously does not satisfy (2.5). In this case, it was first shown [76,77], that no solution satisfying constancy of $M_{1}(t)$ could exist beyond a certain time. This negative result was later put into proper perspective by the discovery $[115,58]$ of an exact solution for all times, for which $M_{1}(t)$ decays after a finite time. This phenomenon, which is found to be linked to the appearance of an infinite cluster at finite time, is known as gelation. It is of considerable importance and we shall discuss it extensively further on in this paper.

This led to a different set of existence results [58], which did not aim to prove either constancy of $M_{1}(t)$ or any decay condition leading to (2.7). It could then be shown in [58] that, if

$$
K\left(m, m^{\prime}\right) \leq r(m) r\left(m^{\prime}\right) \quad r(m)=o(m),
$$

then a solution of (2.2) with finite and monotonically decreasing $M_{1}(t)$ exists for all times, but no statement was made either concerning the conservation of mass or uniqueness of the solution. The latter is certainly unfortunate, but is intimately related to the technique of proof used. This issue has now essentially been solved in [81], where many other striking results are shown. The result claimed is that under the hypothesis (2.9) uniqueness holds. The former is, on the other hand, only to be expected. In fact, as we shall see, the scaling theory strongly suggests that the bound (2.5) is, in fact, exactly the one that separates gelation at finite time from regular behaviour at all times. As a further indication of this fact, the model

$$
K\left(m, m^{\prime}\right)=m^{\alpha} \delta_{m, m^{\prime}}
$$

can be rigorously shown to violate mass conservation if $\alpha>1$ [60], whereas it satisfies (2.5) - and hence mass conservation - if $\alpha \leq 1$. The marginal case $\alpha=1$ is discussed in greater detail in [10].

Let me shortly describe the technique involved, since it turns out to be of fairly general applicability: one first defines a finite, mass-conserving system, 
for which standard results can be invoked to guarantee existence of the solution for all times. A compactness argument is then used to show that the solutions to the finite systems have a convergent subsequence. It can then be shown that the limit of this subsequence satisfies a weak form of (2.2), and finally that it satisfies (2.2) in a strong sense as well. The mode of convergence, however, is not strong enough to conclude that the mass contained in the limiting solution is the same as that in the finite approximants, which is what gives to this approach the requisite generality. The extension to the case of continuously variable $m$ presents some technical difficulties. These were adressed by Bak [3] in the case of a reaction kernel bounded above and below by a constant. The general case was treated by Ball and Carr in [5].

Another extremely important issue from the point of view of applications concerns the extension to non-uniform systems This involves replacing (2.2) by a reaction diffusion equation. This has been treated by Slemrod [88], along lines similar to those sketched above. Here, however, it should be emphasized that even the "standard" results on the finite approximants are highly nontrivial in this case. While the results obtained are remarkable indeed, I believe that they are probably not yet optimal: indeed, the strongest results obtained concern diffusion constants bounded from below and above by a constant. In a realistic situation, however, these always decrease with mass; an extension of these results to more general cases would certainly be very desirable.

Finally, it should be emphasized that the issues adressed here are not altogether idle: existence may indeed fail under some circumstances. There is considerable evidence, for example, that if $K\left(m_{0}, m\right) / m \rightarrow \infty$ as $m \rightarrow \infty$ for fixed $m_{0}$, then either no solutions exist or these solutions have the peculiar property of violating mass conservation from the very start. In this case it is an open problem whether the solution exists at all for a sufficiently wide set of initial conditions. For more details on these fascinating questions, see $[107,16,55,56]$.

\section{$3 \quad$ The Scaling Hypothesis}

\subsection{Basic Concepts: Moments and Typical Size}

The crucial observation underlying the scaling approach is the following: at large times the value of most quantities of interest - namely those that result from an average over the whole cluster size distribution-can be computed using a single "typical size" which grows indefinitely with time. Under these circumstances, we expect that only the coarsest features of the $K\left(m, m^{\prime}\right)$ will be relevant. Let us therefore assume that $K\left(m, m^{\prime}\right)$ is asymptotically homoge- 
neous of degree $\lambda$, that is, that there exist a $\lambda$ and a function $\bar{K}\left(m, m^{\prime}\right)$ such that

$$
\bar{K}\left(a m, a m^{\prime}\right)=\lim _{s \rightarrow \infty}\left[s^{-\lambda} K\left(s m, s m^{\prime}\right)\right]
$$

The function $\bar{K}\left(m, m^{\prime}\right)$ is then clearly homogeneous of order $\lambda$. To describe $\bar{K}\left(m, m^{\prime}\right)$ in greater detail, note that it can be written as

$$
\bar{K}\left(m, m^{\prime}\right)=m^{\lambda} k\left(\frac{m^{\prime}}{m}\right),
$$

where $k(z)$ is a function subject to the symmetry condition

$$
k(z)=z^{\lambda} k(1 / z)
$$

but which is otherwise quite arbitrary. It can therefore be chosen arbitrarily, say, between 0 and 1 . We now define the exponent $\mu$ and the prefactor $\Gamma$ which describe the (power law) behaviour of $k(z)$ near the origin. More specifically:

$$
k(z)=\Gamma z^{\mu}[1+o(1)]
$$

One additionally defines $\nu$ as the exponent describing the behaviour of $k(z)$ at infinity. It follows from (3.3) that

$$
\nu=\lambda-\mu \text {. }
$$

It follows from these definitions that there exist reaction rates corresponding to arbitrary values ${ }^{6}$ of $\mu$ and $\nu$, as evidenced by

$$
K\left(m, m^{\prime}\right)= \begin{cases}m^{\mu} m^{\prime \nu} & \left(m \leq m^{\prime}\right) \\ m^{\nu} m^{\prime \mu} & \left(m \geq m^{\prime}\right)\end{cases}
$$

At this stage, we should rephrase the existence and uniqueness results described in the previous subsection. Condition (2.5) is equivalent to $\lambda \leq 1$ and $\nu \leq 1$. It is indeed presumably optimal for the existence of a global mass-conserving solution of (2.2). On the other hand, condition (2.9) which guarantees existence only, is equivalent to the conditions $\lambda<2$ and $\nu<1$.

$\overline{6}$ This issue has caused some confusion in the literature, as a lot of work has gone into the study of kernels of the form $m^{\alpha} m^{\prime \lambda-\alpha}+m^{\lambda-\alpha} m^{\prime \alpha}$, which do not generate the whole range of possible $\mu$ and $\nu$ values, as opposed to (3.6) 
However, a straightforward extension of the proof given in [58] shows that the former assumption can be dispensed with. The only essential condition is, therefore, $\nu<1 .^{7}$

Let us temporarily assume that $K\left(m, m^{\prime}\right)$ is exactly homogeneous. From this assumption follows [36] that, if $c(m, t)$ is an arbitrary solution of $(2.2)$, then so is

$$
\left(T_{a, b} c\right)(m, t)=a^{\lambda+1} b^{-1} c(a m, b t)
$$

for arbitrary positive $a$ and $b$. The existence of such a symmetry group motivates the search for solutions which remain invariant under it. In other words, we ask whether solutions exist, which, as time evolves, merely transform into similar solutions generated from a constant cluster size distribution by a variable group transformation of the form (3.7). In the so-called regular case, that is, when mass conservation holds, see (2.5) , this can only occur if the group transformation belongs to the subgroup of (3.7) which maintains the total mass in the system invariant, that is $T_{a, a^{\lambda-1}}$. From this follows that such a solution necessarily has the form

$$
c(m, t)=W t^{-2 /(1-\lambda)} \Phi\left(m / t^{1 /(1-\lambda)}\right)
$$

where $W$ is a constant factor which will be of use later ${ }^{8}$. The gelling case, as we shall see below, requires more careful considerations. Quite generally, however, we shall say that a solution is of scaling form if it can be written in the form

$$
c(m, t)=W s(t)^{-2} \Phi\left(\frac{j}{s(t)}\right),
$$

where $s(t)$ is an arbitrary (growing) function of time, which is called the typical size. From this follows, as we show in greater detail later, that if we define the moments of the distribution as follows

$$
M_{p}(t)=\int_{0}^{\infty} m^{p} c(m, t) d m
$$

7 The exponents $\lambda$ and $\mu$ above can be defined in a rather rough manner, except if we consider the limiting cases $\lambda=1$ and $\nu=1$. For these it is essential that there be no logarithmic corrections to the power law behaviour. see e.g. [10] for details.

8 Specialists will recognize here the separation constant $w$ of the papers of Ernst and van Dongen 
one finds as an estimate for the order of magnitude of $s(t)$

$$
s(t) \simeq \frac{M_{p+1}(t)}{M_{p}(t)}
$$

at least when $p$ is larger than a given $p_{0}$, the value of which depends on the details of the system under consideration. In fact, in many contexts, the typical size is defined as some such moment ratio: such are, for example, the weight average, defined as $M_{2}(t) / M_{1}(t)$ and the $z$-average, given by $M_{3}(t) / M_{2}(t)$. The constant $W$ will allow us to maintain any such definitions, while keeping simple normalizations for the function $\Phi(x)$.

We shall show in the following sections that, if such a solution exists, the function $\Phi(x)$ must satisfy a certain integral equation. Conversely, it is straightforward to see that any solution to the integral equation also defines via (3.8) a self-similar solution of (2.2).

The scaling hypothesis now reduces to the following statement: every solution starting from a "narrow" cluster size distribution (that is, one that does not have already some kind of power law distribution at large masses) evolves for large times into a self-similar solution of the type we have just described ${ }^{9}$. We are therefore assuming that these specific solutions, which can be described quite accurately in a broad variety of cases, are in fact the only relevant solutions at large times, as they are approached from essentially any initial conditions. It should be emphasized that there is no proof of this so-called "scaling hypothesis". nor is there any idea of how such a proof might go. However, the numerical evidence for it is good, and we shall see that it is satisfied in all exactly solved models.

\subsection{Different Kinds of Scaling}

In the following, we define more accurately in which sense we expect solutions of (2.2) to tend towards a scaling solution. This issue is of fundamental importance, as it will accurately determine the kind of conclusions one may legitimately draw from scaling and those one may not.

To justify the definition I shall make, let me first state the nature of the object we are interested in: this is the cluster size distribution $c(m, t)$. The information it gives us is the following: how many clusters are there at time $t$ in the mass range going from $m$ to $m+d m$. As such, the cluster size distribution

\footnotetext{
$\overline{9}$ In fact, we shall see that, quite often, even initial conditions with power-law tails will approach the same scaling limit as narrow ones. The above is merely the most conservative formulation of the hypothesis.
} 
is essentially a time-dependent measure on the set of all masses. We therefore need a notion of convergence adapted to measures.

It turns out, as we shall justify in the following, that the appropriate type of convergence is weak convergence. Specifically, we shall say that a cluster size distribution $c(m, t)$ tends to a scaling form if there is a function $s(t) \rightarrow \infty$ as $t \rightarrow \infty$ and such that

$$
\int_{0}^{\infty} m c(m, t) f[m / s(t)] d m \underset{t \rightarrow \infty}{\longrightarrow} W \int_{0}^{\infty} x \Phi(x) f(x) d x
$$

for every continuous bounded function $f(x)$ on the positive real axis. Note that the use of $x \Phi(x)$ in the definition is purely conventional: it serves to make notation consistent with the previous subsection, as well as with ordinary usage.

We shall argue in the following that (3.12) is the most appropriate definition for the appproach to scaling. On the one hand we shall find that the various exactly solved models which we discuss in Section 4 yield counterexamples to many attempts to make sharper statements, whereas (3.12) is, as we shall see in some detail, fulfilled in the vast majority of cases.

Let me here make a technical-but quite important-remark: in order to prove weak convergence of measures, it is necessary and sufficient, by a standard result in probability theory [114], to show that the corresponding Fourier transforms converge. Since all the measures we consider are concentrated on the positive real axis ${ }^{10}$, this is equivalent to convergence of the corresponding Laplace transforms, or generating functions in the discrete case. In other words, if we define

$$
G(\zeta ; t)=\int_{0}^{\infty} c(m, t) e^{\zeta m} d m
$$

we obtain the following two equivalent formal expressions for the scaling function $\Phi(x)$ :

$$
\begin{aligned}
& W \int_{0}^{\infty} \Phi(x)\left(e^{-\rho x}-1\right)=\lim _{t \rightarrow \infty}\{s(t) G[-\rho / s(t), t]\} \\
& x \Phi(x)=W^{-1} \mathcal{L}^{-1}\left\{\lim _{t \rightarrow \infty}\left[G_{\rho}(-\rho /(s(t), t)]\right\}(x),\right.
\end{aligned}
$$

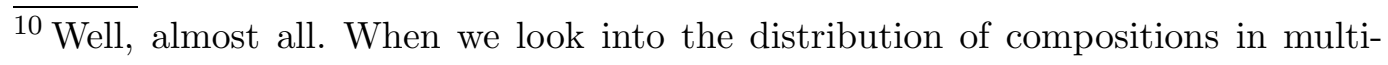
component aggregation, we shall in fact use Fourier transforms 
where $\mathcal{L}^{-1}$ denotes the inverse Laplace transform. Since generating functions such as $G(\zeta, t)$ are ubiquitous in exact solutions, where they arise in a very natural manner, we may already argue from an aesthetic viewpoint in favour of the above defiinition of convergence to scaling.

Finally note that there is one element of arbirariness in the definition (3.12) that I have not yet addressed: it concerns the choice of the measure we decide to consider. In (3.12) we chose $m c(m, t)$, partly because it is automatically normalized. It is, however, quite possible to use, for example, $m^{n} c(m, t) / M_{n}(t)$ in its stead. We shall say that the $n$ 'th moment of the cluster size distribution approaches a scaling form if there is a function $s(t)$ as above such that

$$
M_{n}(t)^{-1} \int_{0}^{\infty} m^{n} c(m, t) f[m / s(t)] d m \underset{t \rightarrow \infty}{\longrightarrow} W \int_{0}^{\infty} x^{n} \Phi(x) f(x) d x
$$

for every continuous bounded function $f(x)$ on the positive real axis. Now, it is straightforward to check that, if the $n$ 'th moment of a cluster size distribution tends to a scaling form, then so do all higher ones. However, the opposite need not be the case. Consider for example the exact solution to (2.2) for $K\left(m, m^{\prime}\right)=m m^{\prime}$ and an initial condition $c(m, 0)=\delta(m-1)$, which is given by

$$
c(m, t)=\sum_{k=1}^{\infty} \frac{k^{k-2}}{k !} \frac{\left(t e^{-t}\right)^{k}}{t} \delta(m-k),
$$

and is valid for $t \leq 1$. In this case, one readily verifies that, choosing $s(t)=$ $(1-t)^{-2}$, the second moment of (3.16) approaches a scaling form with $\Phi(x)$ given by $x^{-5 / 2} e^{-x}$, whereas the first does not tend to a scaling form at all. Generally speaking, the scaling function to which the $n$ 'th moment tends must satisfy

$$
\int_{0}^{\infty} d x x^{n} \Phi(x)<\infty
$$

so that a singularity of the type $x^{-5 / 2}$ could never arise as a limit of first moments.

\subsection{Some Consequences that Follow from Scaling, and More that Don't}

We now need to understand which consequences follow from the scaling hypothesis. In many systems, it is possible to measure some of the moments 
$M_{p}(t)$ of the cluster size distribution $c(m, t)$, as defined in (3.10). In fact, it is often, at least in principle, possible to reconstruct the full distribution from a knowledge of its moments. We therefore concentrate first on these. From the definition of convergence to a scaling form (3.12) follows

$$
\lim _{t \rightarrow \infty}\left[s(t)^{-(p-1)} M_{p}(t)\right]=W \int_{0}^{\infty} x^{p} \Phi(x) d x=W \bar{M}_{p}
$$

whenever the integral on the r.h.s converges. Here the last equality defines the scaled moments $\bar{M}_{p}$ which play a considerable role throughout the theory. As we shall find out later, $\Phi(x)$ has no singularities for $x \neq 0$ and decays exponentially as $x \rightarrow \infty$.

The only issue is therefore the behaviour at zero. In many cases, this is given by a power law as follows

$$
\Phi(x) \simeq x^{-\tau} \quad(x \rightarrow 0) .
$$

The meaning of such a behaviour at the origin is the following: it states that the concentrations averaged in the low end of the cluster distribution go as $m^{-\tau}$. More formally,

$$
\lim _{t \rightarrow \infty}\left[s(t)^{2} \frac{1}{\epsilon s(t)} \int_{\epsilon s(t)}^{2 \epsilon s(t)} c\left(m^{\prime}, t\right) d m^{\prime}\right]=O\left(\epsilon^{-\tau}\right)
$$

as $\epsilon \rightarrow 0$. Here the 1.h.s. is an averaged concentration rescaled by $s(t)^{2}$ so as to cancel the time dependence.

It now follows that a necessary condition for (3.18) to hold is

$$
p>\tau-1
$$

(3.18) and (3.21) therefore confirm the claim made in (3.11) that $s(t)$ can be defined as the ratio of successive moments, if the order of the moments is sufficiently large.

Let us now introduce some notation: denote by $z$ the exponent with which $s(t)$ grows as a function of $t$ and $\alpha_{p}$ the one with which $M_{p}(t)$ grows

$$
\begin{aligned}
s(t) & \simeq t^{z} \\
M_{p}(t) & \simeq t^{\alpha_{p}}
\end{aligned}
$$


From these definitions we obtain the following relationships

$$
\alpha_{p}=(p-1) z \quad(p>\tau-1) .
$$

We may further define $w$ as the exponent with which $c(m, t)$ decays when $s(t)$ becomes much larger than $m$. Formally once more

$$
\lim _{\epsilon \rightarrow 0}\left[\frac{1}{\epsilon s(t)} \int_{\epsilon s(t)}^{2 \epsilon s(t)} c\left(m^{\prime}, t\right) d m^{\prime}\right]=O\left(t^{-w}\right)
$$

From (3.19) and (3.24) one readily shows the following celebrated identity [110]

$$
(2-\tau) z=w .
$$

This relation relates large time behaviour of small clusters to the shape of the cluster size distribution at the low end of the distribution for fixed large times. Since these two quantities are not related in a straightforward way, this relation often gives very useful and non-trivial information.

Note, however, that the above definitions of $\tau$ and $w$ seem quite artificial. It appears much more natural to ask about the large time behaviour of $c(m, t)$ at fixed $m$ and the small $m$ behaviour at fixed $t$. Here, however, it must clearly be stated that these asymptotic behaviours cannot be determined from the scaling hypothesis in the form that we have given it up to now. Similarly, moments $M_{p}(t)$ of order lower than $\tau-1$ cannot be described by scaling theory as it stands. In fact, their behaviour is linked to that of the $c(m, t)$ at fixed $m$ for large $t$, which lies beyond the reach of the scaling approach. We may define some additional exponents: for example, define $\tau^{\prime}$ and $w^{\prime}$ as follows

$$
\begin{aligned}
& c(m, t) \simeq m^{-\tau^{\prime}} \quad(1 \ll m \ll s(t)) \\
& c(m, t) \simeq t^{-w^{\prime}} \quad(t \rightarrow \infty) .
\end{aligned}
$$

At this stage, however, I should again remark that exactly solved models are known (see section 4) for which the $w^{\prime}$ exponent depends on $m$, whereas the $\tau^{\prime}$ exponent does not exist. These, however, satisfy ordinary scaling and have well defined values of the exponents $\tau, z$ and $w$.

Nevertheless, since such systems are not very frequent (they are not, however, pathological), it makes sense to say that a system satisfies the strong scaling hypothesis if $\tau^{\prime}$ and $w^{\prime}$ both exist and satisfy

$$
\tau^{\prime}=\tau \quad w^{\prime}=w .
$$


Under these circumstances, we can say how the moments $M_{p}(t)$ of order $p<p_{0}$ behave if they are finite initially: the $\alpha_{p}$ are given by

$$
\alpha_{p}= \begin{cases}(p-1) z & (p>\tau-1) \\ w & (p \leq \tau-1)\end{cases}
$$

Note, however, that if strong scaling is violated, it may well happen that $\alpha_{p} \neq(p-1) z$, even when $p>\tau-1$, as we shall later show in explicit examples. The only generally true statement is that $\alpha_{p}=(p-1) z$ if $p>1$. Since the evaluation of moments is of central importance in applications of the theory to real systems, this result is basic. It shows that the large time behaviour of moments can always be obtained to a knowledge of the exponents $\tau$ and $z$ for given forms of the reaction kernel $K\left(m, m^{\prime}\right)$. It is this task to which we now turn.

\subsection{The Scaling Equation and Its Derivation}

The basic kinetic equations (2.2) together with the definition (3.8) of the fact that the first moment of $c(m, t)$ approaches a scaling limit, given in (3.12) lead to the following condition on the scaling function $\Phi(x)$ : for all continuous functions on the positive real axis which vanish sufficiently fast at infinity, one has, as shown in Appendix A:

$$
\int_{0}^{\infty} d x d y \bar{K}(x, y) \Phi(x) \Phi(y) x[f(x+y)-f(x)]=\int_{0}^{\infty} d x x^{2} f^{\prime}(x) \Phi(x) .
$$

Here $\bar{K}(x, y)$ is the continuous function defined by

$$
\bar{K}(x, y)=\lim _{s \rightarrow \infty}\left[s^{-\lambda} K(s x, s y) .\right]
$$

As part of the derivation of this equation, one obtains a condition on $s(t)$, namely

$$
\dot{s}(t)=W s(t)^{\lambda}
$$

the solution of which is in agreement with the statements obtained on quite general grounds in (3.8). This therefore confirms that

$$
z=\frac{1}{1-\lambda}
$$


when gelation does not occur. The derivation of (3.29) is somewhat technical and is given in appendix A. We present it in some detail, though, as the scheme is very straightforward and can be extended in a mechanical way to all the other cases we shall be considering in this paper.

A particularly useful form of (3.29) is the one in which $f(x)$ is taken as an arbitrary exponential:

$$
\begin{aligned}
& I(\rho):=\int_{0}^{\infty} d x d y \bar{K}(x, y) \Phi(x) \Phi(y) x e^{-\rho x}\left[1-e^{-\rho y}\right] \\
& I(\rho)=\rho \int_{0}^{\infty} d x x^{2} e^{-\rho x} \Phi(x) .
\end{aligned}
$$

Here $I(\rho)$ is defined for future reference in (3.33) A very similar equation is central to the work of van Dongen [108]. It is obtained from (3.29) by setting $f(x)=\Theta(a-x)$ for arbitrary $a$ and yields the relation

$$
a^{2} \Phi(a)=\int_{0}^{a} d x \int_{a-x}^{\infty} d y x \bar{K}(x, y) \Phi(x) \Phi(y)
$$

I present (3.35) because of its importance in previous work, but shall not make much use of it in the sequel. From (3.29) also follows immediately the following central expression for the scaled moments $\bar{M}_{p}$.

$$
p \bar{M}_{p+1}=\int_{0}^{\infty} d x d y x \bar{K}(x, y) \Phi(x) \Phi(y)\left[(x+y)^{p}-x^{p}\right]
$$

When $\bar{K}(x, y)$ is of the form $x^{\alpha} y^{\lambda-\alpha}$, then (3.36) becomes a nonlinear recursion between the scaled moments whic is frequently useful, see for example subsection 3.5.2. Also, as we shall later show, the scaled moments enter in the determination of $\tau$ for certain cases (see (3.48) below), and (3.36) then turns out to be very useful to get interesting results from these connections.

Finally, let me make some remarks on uniqueness and normalization of the solutions of (3.29) as well as all its special variants. It is clear that (3.29) inherits the symmetry of the equations (2.2), as stated in (3.7). Namely, whenever $\Phi(x)$ is a solution, so is

$$
\Phi_{b}(x)=b^{1+\lambda} \Phi\left(\frac{x}{b}\right)
$$


for all $b>0$. This ambiguity in the definition of $\Phi(x)$ arises from the absence of scale in the equations (2.2) at least when aggregates are large enough and differs from the ambiguity which simply results from the choice of the scale of $s(t)$, which we have absorbed in the prefactor $W$ in (3.12). It should generally be checked that final results are not affected by the transformation (3.37). To fix the normalization, we use the fact that, since $x \Phi(x)$ is defined as the weak limit of a probability distribution, it must be normalized (gelation is not assumed to occur in this case). This means

$$
\int_{0}^{\infty} x \Phi(x) d x=1 .
$$

This condition then determines $b$ in 3.37) and hence $\Phi(x)$ uniquely. Finally, the absolute scale of $\Phi(x)$ is related to that of $\bar{K}(x, y)$, which is itself fixed by the choice of time scale. This can be stated as follows. If $\Phi(x)$ is a solution with rate $\bar{K}(x, y)$, then $b \Phi(x)$ is a solution to the equation with rates $\bar{K}(x, y) / b$.

Note that a slight difficulty arises when $\lambda=1$, since the transformation defined by (3.37) then leaves $\int x \Phi(x) d x$ invariant. In this case we must keep the constant $W_{1}$ explicitly in (A.5) and set the normalization by allowing it to vary.

The above relations were all derived under the hypothesis that the first moment of the cluster size distribution tends to a scaling form. This is not always the case. In fact, it is easy to verify that, when $\tau>2$, it is impossible to have such convergence. Therefore, in the gelling case, which always has $\tau>2$, we must consider convergence of higher order moments. In this case one finds

$$
\begin{gathered}
\int d x\left[(3-\tau) f(x)+x f^{\prime}(x)\right] x^{2} \Phi(x)=\int d x d y \bar{K}(x, y) \times \\
\times \Phi(x) \Phi(y) x[(x+y) f(x+y)-x f(x)]
\end{gathered}
$$

Here we have used the strong scaling assumption to show that

$$
\frac{\dot{M}_{2}(t)}{M_{2}(t)}=(3-\tau) \frac{\dot{s}(t)}{s(t)}
$$

The appearance of the exponent $\tau$ in this equation may at first appear disconcerting, but there is no real problem: (3.39) can be solved for any $\tau$. It then remains to see for which value of $\tau$ the condition (3.19) obtains. We now show how these equations can be used to determine $\tau$ from the knowledge of the exponents $\lambda$ and $\mu$ characterizing the reaction kernel $\bar{K}(x, y)$. 


\subsection{The Solutions of the Scaling Equation}

\subsubsection{The Non-gelling Case: Small-x Behaviour}

A very useful mathematical concept in the determination of the behaviour of $\Phi(x)$ near the origin is that of regular behaviour. We say that a function $\Phi(x)$ has regular behaviour at the origin if the limit

$$
h(a)=\lim _{x \rightarrow 0} \frac{\Phi(a x)}{\Phi(x)}
$$

exists. If this is the case, it is easy to see that a number $\tau$ must exist such that

$$
h(a)=a^{-\tau} .
$$

This is shown by proving that $h(a)$ must satisfy the functional equation

$$
h(a b)=h(a) h(b),
$$

for which power laws are the only solutions ${ }^{11}$.

We now assume that $\Phi(x)$ has regular behaviour near the origin. That is, we assume that it has a power law behaviour, possibly dressed by arbitrary logarithmic corrections, but no behaviour of the type $\cos (b \ln x)$. We shall find that this assumption is self-consistent for $\mu \geq 0$, but that it is not so when $\mu<0$. In the former case it allows us to derive more or less explicit expressions for $z$ and $\tau$. In the latter, we shall see that $\Phi(x)$ has a stretched exponential behaviour near the origin, which can be determined by other techniques. It should be realized, however, that self-consistency does not prove that the function $\Phi(x)$ does display regular behaviour at the origin. In fact, there is substantial numerical evidence [56] that when $\mu>0$ the function $\Phi(x)$ has non-regular behaviour at the origin, with (possibly) undamped logarithmic oscillations.

Since we are interested in small values of $x$, we naturally consider the large- $\rho$ behaviour of the two sides of (3.34). We show in appendix C, see (C.6) and (C.7), that

$$
I(\rho)=B_{1} \rho^{-(\lambda+3)} \Phi(1 / \rho)^{2}+B_{2} \rho^{-2} \Phi(1 / \rho) k(1 / \rho),
$$

where $B_{1}$ and $B_{2}$ are definite integrals defined by (C.7) with the following peculiarity: of the two integrals involved, one will diverge and the other one

\footnotetext{
${ }^{11}$ I disregard non-measurable functions throughout this paper.
} 
converge for any choice of $\Phi(x)$. Only the term with the convergent integral should be retained in (3.44).

From this follows, by matching $I(\rho)$ with the r.h.s. of (3.34) which is of order $\rho^{-2} \Phi(1 / \rho)$, the following well-known three cases (the numbers have become standard terminology):

- Case I: $\mu>0$. For these values of the parameters one finds

$$
\tau=1+\lambda \quad w=1 .
$$

In this case, however, there exists some doubt on the very existence of a well-defined solution to (3.29). The issue is the following: it is readily seen that the function $x^{-(\lambda+1)}$ solves (3.29) quite generally. However, it clearly never fulfils the essential normalization condition (3.38). This means that the condition of strong decay as $x \rightarrow \infty$ is essential in determining the solution. This has cast some doubt on the existence of such a solution. On the other hand, recent numerical work by Lee (see [56]) strongly suggests that such a solution does exist ${ }^{12}$, though it may not behave regularly at the origin: specifically, Lee observes oscillations in $\Phi(x)$, which might well be of the type $\cos \ln x$. These do not conform to the regularity hypothesis. On the other hand, if $\Phi(x)$ really does behave regularly at the origin, we may state that

$$
\Phi(x)=B_{1} x^{-\tau}
$$

where $B_{1}$ is given by (C.7), which is a definite integral that can be explicitly evaluated for every single kernel.

Finally note that the value for $w$ is the lowest possible for the monomer decay exponent in a system with binary reactions, since $c_{1}(t)$ can always decay at least via a reaction with itself.

- Case II: $\mu=0$. In this case, the exponent $\tau$ is non-universal, but satisfies the inequality

$$
\tau<1+\lambda
$$

If $\Phi(x)$ behaves regularly at the origin, then a comparison of the prefactors of the r.h.s. and l.h.s. of (3.29) yields the well-known relation [108]

$$
\tau=2-\Gamma \int_{0}^{\infty} x^{\lambda} \Phi(x) d x=2-\Gamma \bar{M}_{\lambda},
$$

where $\Gamma$ is defined by (3.4) and $\bar{M}_{p}$ is defined as in (3.18). Note that this result is invariant under the symmetry transformation (3.37) as well as under a common scale change of $\Phi$ and $\bar{K}$ (due to the presence of $\Gamma$ ).

\footnotetext{
$\overline{12}$ contradicted, however, by earlier work, see in particular [53]
} 
Of course, (3.48) does not straightforwardly determine $\tau$, since it requires in principle the knowledge of the full scaling function $\Phi(x)$, from which the exponent $\tau$ could have been derived without recourse to (3.48). However, as has been shown in $[108,18],(3.48)$ can be used to determine bounds on $\tau$ for any specific kernel independently of $\Phi(x)$. In particular, Cueille and Sire [18] have developed a large number of very elegant methods which allow to obtain extremely sharp rigorous upper and lower bounds on $\tau$, to the extent that one may view these as the equivalent of an exact determination. In some specific cases, $\tau$ can also be displayed as the solution to a transcendental equation [102].

- Case III: $\mu<0$. In this case, the assumption of regular behaviour of $\Phi(x)$ near the origin leads to inconsistencies. We may therefore assume a decay of $\Phi(x)$ near the origin that is faster than every power. One may then approximate $I(\rho)$ as shown in appendix $\mathrm{C}$, with the result

$$
I(\rho)=\Gamma \bar{M}_{\lambda-\mu} \int_{0}^{\infty} x^{1+\mu} \Phi(x) e^{-\rho x} d x .
$$

From this and (3.34) follows, by inverting Laplace transforms, that within our approximations

$$
\left[x^{2} \Phi(x)\right]^{\prime}=\Gamma \bar{M}_{\lambda-\mu} x^{1+\mu} \Phi(x) .
$$

From this one finds the approximate form for $\Phi(x)$ near the origin in this case:

$$
x^{2} \Phi(x)=\text { const. } \cdot \exp \left[-\Gamma \bar{M}_{\lambda-\mu} \frac{x^{-|\mu|}}{|\mu|}\right]
$$

Here only the exponential behaviour with the constants indicated are reliable. The power law $x^{-2}$ does not follow at the degree of accuracy we have used here. For a detailed treatment of corrections to scaling for both Case II and Case III, see [108]. It is an entertaining excercise for the reader to verify that (3.51) is indeed invariant under the symmetry transformations (3.37) as well as under a simultaneous change of scale in $\bar{K}$ and $\Phi(x)$.

Yet another dificulty arises when $\lambda=1$ and $\mu>0$ : in this case, since $\tau$ is predicted by (3.45) to be two, the normalization condition (3.38) for $\Phi(x)$ is not satisfied any more. Under these circumstances the easiest solution is presumably to view this as an instance where it is more appropriate to look at the convergence of the second moment to a scaling form, so we defer consideration of this issue to the next subsection, in which we consider also the gelling case, which cannot be treated in any other way. For a different approach, however, see [108]. On the other hand, if $\mu \leq 0$, we find ourselves either in Case II or in Case III. Due to inequality (3.47) in Case II and (3.51) in Case III, no convergence problems arise even when $\lambda=1$. 


\subsubsection{The Non-gelling Case: Large-x Behaviour}

The large- $x$ behaviour in the non-gelling case has been treated by van Dongen and Ernst in [106,108]. We present here a slightly modified version of their approach. Let us first show that whenever $K\left(m, m^{\prime}\right)$ is less than $m+m^{\prime}$, the scaling function $\Phi(x)$ decays exponentially for large values of $x$. This can be done as follows: consider the moments $\bar{M}_{p}$ of the scaling function. These satisfy the relation (3.36), which implies, using the bound on the kernel $K\left(m, m^{\prime}\right)$

$$
\bar{M}_{p+1} \leq \frac{1}{2 p} \sum_{k=1}^{p}\left(\begin{array}{l}
p \\
k
\end{array}\right) \bar{M}_{k} \bar{M}_{p-k+1} .
$$

If we now denote by $\bar{M}_{p}^{(0)}$ the moments which satisfy (3.52) as an equality, it is easy to show inductively that

$$
\bar{M}_{p} \leq \bar{M}_{p}^{(0)}
$$

for all $p$. On the other hand, it is seen that the $\bar{M}_{p}^{(0)}$ can be evaluated analytically, since they are the moments of the scaling function of the kernel $m+m^{\prime}$, which is known exactly. One therefore obtains

$$
\bar{M}_{p} \leq \bar{M}_{p}^{(0)}=\text { const. } \cdot p^{-3 / 2} p ! R^{p}
$$

for all $p$ and some fixed value of $R$, which strongly depends on various normalizations. (3.54) now immediately leads to the desired conclusion by using standard results on the moment problem. Similar lower bounds can also be shown assuming correponding lower bounds on $K\left(m, m^{\prime}\right)$.

Assuming some such lower bound, it follows that the integral equation (3.34) also holds for negative values of $\rho$, up to a critical value $-\rho_{c}$ beyond which all integrals diverge. Introducing

$$
h(x)=\Phi(x) e^{\rho_{c} x}
$$

one obtains the following equation from (3.34) for $h(x)$

$$
\begin{aligned}
& \int_{0}^{\infty} d x x^{2} h(x) e^{-\left(\rho+\rho_{c}\right) x}=-\rho \int_{0}^{\infty} d x d y x K(x, y) h(x) h(y) e^{-\left(\rho+\rho_{c}\right)(x+y)} \times \\
& \times\left[1-e^{\rho y}\right]
\end{aligned}
$$


If we now assume that $h(x)$ behaves regularly at infinity with exponent $\vartheta$, that is,

$$
\lim _{x \rightarrow \infty} \frac{h(a x)}{h(x)}=a^{-\vartheta}
$$

then one obtains, using altogether the same techniques as for the small- $x$ behaviour, that if $\nu<1$

$$
\vartheta=\lambda
$$

If, on the other hand, $\nu=1$, then the integral on the l.h.s of (3.56) diverges. A treatment entirely similar to the one corresponding to the indeterminate Case II for the small $x$ behaviour shows that the exponent $\vartheta$ in this case also cannot be determined uniquely using only the type of scaling considerations discussed above. Details on this subject are to be found in [106].

\subsubsection{The Dominant Singularity Hypothesis}

The following scenario for the explanation of scaling may, at this stage, quite possibly have occurred to the thoughtful reader: if we consider the generating function (or the Laplace transform) of the cluster size distribution function $G(\zeta, t)$ defined by

$$
G(\zeta, t)=\int_{0}^{\infty} c(m, t) e^{\zeta m} d m
$$

one may assume that the appearance of a unique divergent size $s(t)$ in the cluster size distribution $c(m, t)$ might translate itself in the appearance of a single, dominant singularity of $G(\zeta, t)$ on the positive real axis. Certainly, standard theorems on Laplace transforms state that the nearest singularity does indeed lie on the positive real axis, so that such a hypothesis may seem plausible. This assumption might be further strengthened by the careful study of a large number of exactly solved models, since indeed the mechanism explaining the growth of the typical size and the appearance of scaling is invariably of this nature.

Let us now use our understanding of the nature of the scaling function $\Phi(x)$ in order to assess this possibility. Put in equations, our assumption states:

$$
G(\zeta, t)=\text { const. } \cdot\left[\zeta_{c}-\zeta s(t)\right]^{\vartheta+1}+\text { higher order terms, }
$$

where $\zeta_{c}$ is some appropriate constant. 
I have denoted the relevant exponent by $\vartheta$ because, as we shall now see, it has always that value. Indeed, we know from (3.14) that

$$
\int_{0}^{\infty} x \Phi(x) e^{-\zeta x}=\left.\frac{\partial G}{\partial \zeta}(\zeta, t)\right|_{\zeta=-\rho / s(t)}
$$

The large $x$ behaviour of $\Phi(x)$ is given, as is well-known from general theorems on Laplace transforms, by that of the nearest singularity of $G(\zeta, t)$. This nearest singularity is on the real axis, and we have assumed it to be a power-law singularity. It therefore certainly always describes the value of the $\vartheta$ exponent.

Does it also yield the value of the $\tau$ exponent? Clearly, this depends somewhat on the meaning we give to the phrase "higher order terms" in (3.60). However, generally speaking, if we have assumed only one singularity dominating the whole picture, it is hard to see how it could be otherwise. One therefore deduces from the hypothesis just described that

$$
\tau=\vartheta
$$

But this, we know from subsection 3.5.2 is simply wrong in the large majority of cases. Thus, when $\mu>0, \tau=1+\lambda$, which cannot be equal to $\vartheta$, which is $\lambda$. The only cases in which this possibility exists are the loci $\mu=0$, for which $\tau$ is non-universal, and $\nu=1$ for which $\vartheta$ is non-universal. It is certainly striking that all the exactly solved models known to this day find themselves on these loci, and that they indeed satisfy (3.62). This should therefore serve as a warning: some features of exactly solved models are highly atypical.

In subsection 4.10 we shall see that the constant kernel with production term provides a counterexample to the dominant scaling hypothesis. In this case, as will be shown later, one has $\tau=3 / 2$ and $\vartheta=0$. The generating function has infinitely many simple poles tending simultaneoulsy to the origin as $t \rightarrow \infty$. The nearest such pole dominates the large mass behaviour and leads to $\vartheta=0$, whereas the combined effect of all the poles together upon values of $\zeta$ just below zero leads to a $-3 / 2$ singularity.

\subsubsection{The Gelling Case: Scaling Region}

In the gelling case, we define scaling using the approach to scaling of second moments. This is appropriate as long as $\tau<3$. In order to have no difficulty on that score, we limit ourselves in the following to the following range of parameters

$$
1<\lambda \leq 2 \quad \nu \leq 1
$$


The condition $\lambda>1$ is necessary to ensure gelation, whereas the condition $\nu \leq 1$ is required to avoid pathologies such as instantaneous gelation or nonexistence of solutions. The condition which we really only put in for convenience is $\lambda \leq 2$.

Our definition of scaling is therefore the following, there exist a typical size $s(t)$ diverging at the gel time $t_{c}$ and a scaling function $\Phi(x)$ such that

$$
\lim _{t \nearrow t_{c}}\left[\frac{1}{M_{2}(t)} \int_{0}^{\infty} m^{2} c(m, t) f\left(\frac{m}{s(t)}\right) d m\right]=W \int_{0}^{\infty} x^{2} \Phi(x) f(x) .
$$

One now proceeds as in the non-gelling case to derive an integral equation for $\Phi(x)$. The details are carried out in Appendix F. If we now define

$$
s(t)=\text { const. } \cdot\left(t_{c}-t\right)^{-1 / \sigma}
$$

It then follows from the work done in Appendix F that

$$
\sigma=1+\lambda-\tau
$$

In other words, it is no more possible to fix the exponent describing the growth of the typical size in terms of the exponents describing the kernel. Instead, we have a scaling relation between $\sigma$ and $\tau$. Finally, for the integral equation determining $\Phi(x)$, one finds

$$
\begin{gathered}
\int_{0}^{\infty} d x d y x K(x, y) \Phi(x) \Phi(y)[(x+y) f(x+y)-x f(x)]= \\
\int_{0}^{\infty} d x x^{2} \Phi(x)\left[(3-\tau) f(x)+x f^{\prime}(x)\right]
\end{gathered}
$$

The novel feature here again is, of course, the appearance of $\tau$ in the equation. At the basic level, presumably, it does not change much: one can, in principle, solve (3.67) for each value of $\tau$ and then see for which values of $\tau$ (if any) the corresponding solution, which we may call $\Phi_{\tau}(x)$, behaves as $x^{-\tau}$ near the origin.

The difficulty of a theoretical treatment, however, is thereby considerably increased. Let us first ask whether a formal solution of pure power-law form exists, akin to the $x^{-(1+\lambda)}$ solution in the non-gelling case. Let $x^{-\tau}$ be such a solution. In this case, it follows using partial integration that the r.h.s. of 
(3.67) vanishes identically for arbitrary functions. Hence, putting $f(x)=e^{-x}$,

$$
\int_{0}^{\infty} d x d y K(x, y) x^{1-\tau} y^{-\tau} e^{-x}\left[(x+y) e^{-y}-x\right]=0 .
$$

It is a straightforward exercise in gamma functions to show that, for $K(x, y)$ of the form $x^{\mu} y^{\nu}+x^{\nu} y^{\mu}$, the solution of (3.68) is

$$
\tau=\frac{\lambda+3}{2}
$$

Since, however, every kernel of homogeneity degree $\lambda$ can be expressed in terms of kernels of this form, it would appear to follow, at least on a formal level, that this value of $\tau$ is the one that corresponds to the universal powerlaw solution for arbitrary $K(x, y)$. As we shall see in the next subsection, this value is indeed highly significant: it is the only large-size behaviour compatible with a finite flow rate of mass to infinity. As such, it is the only possible largemass exponent for times beyond the gel point. However, we are interested in the scaling region immediately before the gel point. There is, in principle, no reason to assume that this particular value of $\tau$ plays any role in this region.

Let us now attempt a scaling theory of the type we have achieved for the non-gelling case. Our results, unfortunately, will be much more fragmentary than in the regular case. As before, we use exponentials $e^{-\rho x}$ as functions $f(x)$ and investigate the large $\rho$ behaviour. Let us assume that $\Phi(x)$ is of the form

$$
\Phi(x)=A x^{-\tau}\left[1+B x^{\Delta}+o\left(x^{\Delta}\right)\right]
$$

where we have taken next-to-leading behaviour of $\Phi(x)$ into account, since this is what determines the behaviour of the r.h.s. of (3.67). One then obtains, under the hypothesis that the integral (3.68) is different from zero, after some tedious but straightforward calculations

$$
\Delta=1+\lambda-\tau=\sigma .
$$

On the other hand $\tau$ cannot be determined by straightforward scaling arguments. Thus we have a general relation between the first correction to scaling and the growth of the typical size, but neither can be determined, both depending on the exponent $\tau$ which must be determined by other considerations. It is not clear to me whether similar techniques to the ones that were successful in determining $\tau$ to very high accuracy in Case II of the non-gelling case $[18,19]$ may be generalized to this case.

There are therefore two cases to be distinguished: 
(1) The ordinary case: The integral (3.68) is different from zero. In this case we know nothing about the actual value of the exponent $\tau$, but we know how both $\sigma$ and the leading next order correction depend on it. The positivity of $\Delta$, which follows from its definition, leads to the inequality for $\tau$

$$
2<\tau<1+\lambda
$$

(2) The extraordinary case: The integral (3.68) vanishes. In this case we have the "standard" value (3.69) for $\tau$. The computation of the correction to scaling exponents is somewhat more difficult, whereas the exponent $\sigma$ is given by

$$
\sigma=\frac{\lambda-1}{2} .
$$

The nomenclature used above is meant to suggest that the ordinary case is more common than the extraordinary. While I have no analytic arguments to show it, the following evidence exists: first, Lee [56] has studied a large number of gelling kernels of the form $x^{\mu} y^{\nu}+x^{\nu} y^{\mu}$, and has determined $\tau$ numerically to high accuracy. In no instance except the case $\mu=\nu=1$ was $\tau$ given by the "standard" value (3.69) but was always smaller. Second, even in the bilinear kernel, it can be shown (see Appendix L) that the case of power-law initial conditions leads to continuously variable values of $\tau$ as well as an exponent $\Delta$ satisfying (3.71). Therefore, even the bilinear kernel sometimes yields the ordinary case. On the other hand, the extraordinary case really does occur for the bilinear kernel with initial conditions decaying rapidly with mass, since then $\Delta=1$, which contradicts (3.71) with $\lambda=2$ and $\tau=5 / 2$.

In principle, it would now be necessary to derive the value of $\tau$ in the ordinary case from the overall structure of the reaction constants $K\left(m, m^{\prime}\right)$. As far as I can see, this problem is of considerable difficulty, and I have made no headway at all. Possibly the methods developed in $[18,19]$ could be generalized to yield sharp upper and lower bounds on the $\tau$ exponent. This is, of course, particularly important since all other exponents are expressed in terms of $\tau$ and are not known explicitly. Lacking such a theory, we are led back to the numerical study either of the gelation transition in the original equation (2.2) or else of the scaling function $\Phi(x)$ in (3.67).

Finally, let us note that the ordinary case can be characterized in an amusing way as follows: consider the average concentrations defined as follows

$$
\bar{C}(\epsilon)=\frac{1}{\epsilon s(t)} \int_{\epsilon}^{2 \epsilon} c(m, t) d m .
$$


It is then a straightforward consequence of the definition of scaling and of the exponent $\tau$, that

$$
\bar{C}(\epsilon)=\text { const. } \cdot[\epsilon s(t)]^{-\tau}
$$

Now, it is easy to verify that the value of the correction to scaling exponent $\Delta$ given by (3.71) corresponds exactly to the fact that this limiting behaviour is reached linearly in time. This only holds in the ordinary case, so that it should be violated in the case of the bilinear kernel with initial conditions that decay rapidly as a function of mass, as indeed it is: there the average concentrations are readily seen to tend to their limit quadratically in time.

\subsubsection{The Gelling Case: After Gelation}

After gelation sets in, the power-law behaviour of the cluster size distribution remains for all times. Since, by definition, mass must continually decrease after gelation, the derivative must be a finite number, that is,

$$
0<\lim _{M \rightarrow \infty} \frac{d}{d t} \int_{0}^{M} m c(m, t) d m<\infty .
$$

But one finds

$$
\frac{d}{d t} \int_{0}^{M} m c(m, t) d m=\int_{0}^{M} d m_{1} \int_{0}^{\infty} d m_{2} \Theta\left(m_{1}+m_{2}-M\right) \times
$$

If one now inserts the power-law profile $m^{-\tau_{s}}$ for $c(m, t)$ in $(3.77)$, one finds that

$$
\frac{d}{d t} \int_{0}^{M} m c(m, t) d m=\text { const. } \cdot M^{\lambda+3-2 \tau_{s}}
$$

from which follows that, in order for (3.76) to hold, $\tau_{s}$ must indeed have the "standard" value discussed in the previous subsection.

Concerning the large-time behaviour of the concentrations, it can be shown rather generally that they all go as $t^{-1}$. In the discrete case, this may be seen as follows: consider first the monomer equation

$$
\dot{c}_{1}=-c_{1} \sum_{k=1}^{\infty} K(1, k) c_{k} \simeq-c_{1} M_{\nu}(t) .
$$


Since $\tau-\nu>1$, the sum in $M_{\nu}(t)$ converges, so that this quantity behaves similarly to $c_{1}(t)$. Equation (3.79) then reduces to

$$
\dot{c}_{1}=- \text { const. } \cdot c_{1}^{2}
$$

which has the stated behaviour. The general statement then follows by induction, since it is straightforward to show that the production terms are of order $t^{-2}$. Since, however, the removal terms must dominate, they must be of order $t^{-2}$ as well, implying the stated result.

In fact, it is seen that the ansatz

$$
c_{j}(t)=\frac{a_{j}}{t+t_{0}}
$$

can be made to satisfy $(2.2)$ if the $a_{j}$ satisfy the following algebraic relations

$$
a_{j}=\frac{1}{2}\left[\sum_{k=1}^{\infty} K(j, k) a_{k}-1\right]^{-1} \sum_{k=1}^{j-1} K(k, j-k) a_{k} a_{j-k}
$$

It turns out to be quite hard to know whether these equations indeed have a positive solution. For the case in which $K(k, l)$ is of the form $(k l)^{\lambda / 2}$, considerable progress can be made by reducing (3.82) to a recursion together with a self-consistency condition. It was shown [60] that in this case the $a_{j}$ exist and that for large $j$ they display a power-law behaviour $j^{-\tau_{s}}$ if $\lambda>1$. If $0<\lambda<1$, these solutions presumably describe the behaviour of $c_{j}(t)$ at fixed $j$ and $t$ large. They behave for large $j$ as $j^{-(1+\lambda)}$, in agreement with the results derived previously for Case I.

\subsubsection{The Non-Gelling Case for $\lambda=1$}

As has been pointed out above, if $\lambda=1$ and $\nu<1$, the ordinary scaling theory suggests $\tau=2$. This, as first pointed out by van Dongen and Ernst [108], is a contradiction, since one then has a divergent total mass at the origin. The way out is to consider this as a borderline case and treat it via the convergence in second moment, in the same way as we have already done with gelation.

The details are carried out in Appendix F and lead to the following relations

$$
\begin{aligned}
s(t) & =\text { const. } \cdot \exp (\text { const. } \cdot \sqrt{t}) \\
M_{2}(t) & =\frac{s(t)}{\ln s(t)}
\end{aligned}
$$


Convergence to scaling then takes place as follows

$$
\lim _{t \rightarrow \infty}\left[\sqrt{t} e^{-\sqrt{t}} \int_{0}^{\infty} m^{2} c(m, t) f\left(m e^{-\sqrt{t}}\right)\right] d m=\int_{0}^{\infty} x^{2} \Phi(x) f(x) d x .
$$

One may then absorb one factor of $m$ into the function $f\left(m e^{-\sqrt{t}}\right)$ and obtains

$$
\lim _{t \rightarrow \infty}\left[\sqrt{t} \int_{0}^{\infty} m c(m, t) f\left(m e^{-\sqrt{t}}\right)\right] d m=\int_{0}^{\infty} x \Phi(x) f(x) d x
$$

The function $\Phi(x)$ must then satisfy the following integral equation derived in Appendix $\mathrm{F}$

$$
\begin{gathered}
\int_{0}^{\infty} d x d y x K(x, y) \Phi(x) \Phi(y)[(x+y) f(x+y)-x f(x)]= \\
\int_{0}^{\infty} d x x^{2} \Phi(x)\left[f(x)+x f^{\prime}(x)\right]
\end{gathered}
$$

As in the gelling case, $\Phi(x)$ need not have finite mass. This means that, as in the gelling case, the total mass contained in the scaling region vanishes.

\subsection{Crossover}

Up to now, we have mainly considered the case in which the reaction rate $K\left(m, m^{\prime}\right)$ are homogeneous in the masses. The justification of this hypothesis lies, as stated in the Introduction, in the fact that we are principally interested in the behaviour of the system at large times and for large aggregate sizes. Arguably, in such circumstances, any simple physical mechanism for aggregation will have a simple behaviour with respect to scaling of the masses in this asymptotic regime. On the other hand, if two mechanisms act jointly, we may expect that the one that scales with the lesser homogeneity degree will be altogether negligible, so that we may, again, limit ourselves to the homogeneous case. These considerations already suggest, however, a possibly important exception: Assume a fast mechanism which only becomes appreciable at quite large sizes, superimposed on another mechanism, with a lesser

degree of homogeneity, but effective at all sizes. The following illustrates such a possibility:

$$
K\left(m, m^{\prime}\right)=K_{1}^{\left(\lambda_{1}\right)}\left(m, m^{\prime}\right)+\epsilon K_{2}^{\left(\lambda_{2}\right)}\left(m, m^{\prime}\right)
$$


Here the $\lambda_{1,2}$ are two different degrees of homogeneity and $\epsilon$ is a very small number. Under these circumstances, if $\lambda_{2}<\lambda_{1}$, the second term in the sum can obviously be neglected, but in the opposite case, it will eventually dominate. From the point of view of pure scaling thery, it is in principle sufficient to say this, and one has, indeed, a valid prediction for "sufficiently long" times. For practical purposes, however, this is often an excessive limitation, and one would like a scaling theory which gives correct results when times are large with respect to the scale determined by $K_{1}^{-1}$ as opposed to $\epsilon^{-1}$. To this end, we need a theory of crossover.

To avoid unnecessary difficulties, we first fix the scale of the parameter $\epsilon$ : indeed, it is obviously irrelevant whether we use $\epsilon$ or $f(\epsilon)$ as a crossover parameter, where $f$ represents an arbitrary monotonic function vanishing at the origin. In order to fix the parametrization, I therefore make the following convention

$$
K\left(a m, a m^{\prime} ; \epsilon / a\right)=a^{\lambda} K\left(m, m^{\prime} ; \epsilon\right)
$$

At a naive level, this means that $\epsilon$ has a dimension of inverse mass, that is, the natural scale on which $\epsilon$ varies is given by $s(t)^{-1}$. This requirement does not determine $\lambda$ uniquely, though: indeed, replacing $K$ by $K^{\prime}=\epsilon^{\alpha} K$ changes $\lambda$ to $\lambda+\alpha$ while respecting (3.88). We therefore impose the additional requirement that $K(1,1)$ always remain of order one. Thus, for example, the kernel given in (3.87) is rewritten as

$$
K\left(m, m^{\prime}\right)=K_{1}^{\left(\lambda_{1}\right)}\left(m, m^{\prime}\right)+\epsilon^{\lambda_{2}-\lambda_{1}} K_{2}^{\left(\lambda_{2}\right)}\left(m, m^{\prime}\right)
$$

Note that this only gives a meaningful result if $\lambda_{1}<\lambda_{2}$, as was to be expected on intuitive grounds anyway.

We now proceed as always: let us first define what we mean by a scaling limit. This is given by

$$
\lim _{t \rightarrow \infty} \int_{0}^{\infty} m^{\prime} c\left(m^{\prime}, y / s(t) ; t\right) f\left(\frac{m^{\prime}}{s(t)}\right) \rightarrow W \int_{0}^{\infty} x \Phi(x, y) d x
$$

where $c(m, \epsilon ; t)$ denotes the value of the concentration of clusters of mass $m$ at time $t$ evolving under a dynamics using a kernel with a fixed value $\epsilon$ of the crossover parameter. We now use the standard approach in order to derive the following equation for the typical size and the scaling function. For the typical size $s(t)$ one obtains

$$
\dot{s}(t)=W s(t)^{\lambda}
$$


where $\lambda$ is defined by (3.88). Note that, for the case described by (3.89), this means that the degree of homogeneity is $\lambda_{1}$, that is the lesser degree of homnogeneity. From this follows that we will be looking at the size distribution on a scale defined altogether by the slow aggregation process defined by $K_{1}^{\left(\lambda_{1}\right)}$. This may at first appear strange, but it should be noted that, in the scaling limit (3.90), the crossover parameter $\epsilon$ goes to zero, so that the large-time behaviour of any specific system is not the one observed in the crossover limit.

For the scaling function, we obtain the following equation in exactly the same way as we obtained (3.29):

$$
\begin{aligned}
& \int_{0}^{\infty} d x_{1} d x_{2} x_{1} K\left(x_{1}, x_{2} ; y\right) \Phi\left(x_{1}, y\right) \Phi\left(x_{2}, y\right) \times \\
& \quad \times\left[f\left(x_{1}+x_{2}\right)-f\left(x_{1}\right)\right]=\int_{0}^{\infty} d x x f(x)\left[y \Phi_{y}(x, y)-x \Phi_{x}(x, y)-2 \Phi(x, y)\right],
\end{aligned}
$$

which must hold for all continuous function $f(x)$ and for all $y$. From this we again derive an equation involving exponentials similar to (3.34):

$$
\begin{aligned}
\int_{0}^{\infty} d x_{1} d x_{2} x_{1} e^{-\rho x_{1}} K\left(x_{1}, x_{2} ; y\right) \Phi\left(x_{1}, y\right) \Phi\left(x_{2}, y\right) \times \\
\times\left[1-e^{-\rho x_{2}}\right]=\int_{0}^{\infty} d x x e^{-\rho x}\left[2 \Phi(x, y)+x \Phi_{x}(x, y)-y \Phi_{y}(x, y)\right]
\end{aligned}
$$

If one now takes the limit of large $\rho$ and makes exactly similar considerations as in the case without crossover, it is straightforward to show that the exponent $\tau$ defined by

$$
\lim _{a \rightarrow 0} \frac{\Phi(a x, y)}{\Phi(a, y)}=x^{-\tau}
$$

where $y$ is taken to be fixed, is the same as for the kernel $K\left(x_{1}, x_{2} ; 0\right)$, that is, it is identical to the value which would be established if the fast mechanism dominating at long times did not exist. In other words, the small size end of the cluster size distribution is not affected by the existence of the fast aggregation mechanism in the crossover limit as defined by (3.90). Again, this is not at variance with the prediction that the whole size distribution will eventually be determined by the fast process: as time goes on, at fixed $\epsilon$, the value of $y$ diverges and the range of $x$ for which the exponent $\tau$ of the slow process yields a good description, tends to zero. 
The general properties of (3.94) are not easy to work out, however. It is seen that there is a formal solution of the form

$$
\Phi(x, y)=y^{\lambda+1} \chi(x y)
$$

where $\chi(x)$ is some function satisfying an equation which is readily obtained from (3.94). This equation, however, turns out to have no meaningful solutions, since setting $\rho$ to zero in it leads to a contradiction. The solution given in (3.95) must therefore be viewed as a purely formal one, similar to the solution of the form $\Phi(x)=x^{-(\lambda+1)}$ in the usual theory.

Frequently, in practice, one is interested in the time evolution of the moments of the size distribution function, since these are often the only accessible quantities. In this case, if the system is at a fixed value of $\epsilon$, the resulting expression is given by

$$
M_{p}(t ; \epsilon)=W \int_{0}^{\infty} x^{p} \Phi(x, \epsilon s(t)) d x
$$

where $s(t)$ is given by the solution to (3.91). Note, of course, that under these circumstances it is not possible to define $s(t)$ via moment ratios, as is otherwise often done.

\subsection{Extensions of the Model}

\subsubsection{Monomer Production and Diffusion}

In many situations, it happens that monomers are continuously supplied to the system, so that total mass is not conserved any more. If this lasts only for a short time, the evolution of the system after the monomer injection can be viewed as ordinary aggregation starting from a somewhat polydisperse initial condition. This case is therefore not essentially new. If, on the other hand, the supply of monomer takes place in such a way that the total mass supplied diverges, new phenomena arise.

Let us specifically look at the following equations also considered by various authors, see in particular [73,74] and references therein, as well as [20,57]:

$$
\begin{aligned}
\partial_{t} c(m, t)= & \int d m_{1} d m_{2} K\left(m_{1}, m_{2}\right) c\left(m_{1}, t\right) c\left(m_{2}, t\right) \times \\
& \times\left[\delta\left(m_{1}+m_{2}-m\right)-\delta\left(m_{1}-m\right)-\delta\left(m_{2}-m\right)\right]+ \\
& +p\left(t_{0}+t\right)^{\omega} \delta\left(m-m_{0}\right) .
\end{aligned}
$$


Here the time shift $t_{0}$ is introduced merely in order to avoid spurious divergences if $\omega$ is negative. From (3.97) one immediately obtains for the total mass, assuming that gelation does not take place, that is, that $\lambda \leq 1$,

$$
\int_{0}^{\infty} d m m c(m, t)=\frac{p m_{0}\left(t_{0}+t\right)^{1+\omega}}{1+\omega}
$$

From this follows the following natural analogue to the scaling definition (3.12)

$$
\frac{1+\omega}{p m_{0}\left(t_{0}+t\right)^{1+\omega}} \int_{0}^{\infty} m c(m, t) f[m / s(t)] d m \underset{t \rightarrow \infty}{\longrightarrow} W \int_{0}^{\infty} x \Phi(x) f(x) d x
$$

From this we may again, using methods entirely similar to those employed in Appendix A, obtain the following result for the typical size $s(t)$ :

$$
s(t) \approx(W t)^{(\omega+2) /(1-\lambda)} .
$$

For the scaling function $\Phi(x)$ one obtains the following relation similar to (3.29):

$$
\begin{aligned}
& \frac{1+\omega}{W} \int_{0}^{\infty} d x x \Phi(x) f(x)+\int_{0}^{\infty} x^{2} \Phi(x) f^{\prime}(x) d x=\int_{0}^{\infty} d x d y K(x, y) x \Phi(x) \Phi(y) \times \\
& \quad \times[f(x+y)-f(x)]+W^{-2} f(0),
\end{aligned}
$$

If one now substitutes $f(x)$ by $e^{-\rho x}$ one obtain in the limit of large $\rho$ the following condition for $\Phi(x)$ :

$$
I(\rho)=W^{-2},
$$

where $I(\rho)$ is defined by (3.34). If we now use the results of Appendix $\mathrm{C}$ on the large $\rho$ asymptotic behaviour of $I(\rho)$, we find

$$
\tau= \begin{cases}\frac{\lambda+3}{2} & (\nu \leq \mu+1) \\ \mu+2 & (\nu \geq \mu+1)\end{cases}
$$

Assuming strong scaling we can state how the small clusters behave. If $\nu \leq$ $\mu+1$ one finds

$$
c_{j}(t) \approx j^{-(\lambda+3) / 2} t^{\omega / 2}
$$


as stated in [20]. In particular, if $\omega=0$, that is, if monomers are injected into the system at a constant rate, one approaches a stationary distribution with a power-law exponent $(\lambda+3) / 2$. That this value is ubiquitous when a stationary state is reached is well kmown (see e.g. [113]). On the other hand, if $\nu \geq \mu+1$, one finds

$$
c_{j}(t) \approx j^{-(\mu+2)} t^{(\mu+1-(\omega+1) \nu) /(1-\lambda)}
$$

which leads to decaying solutions when $\omega=0$. Therefore, in this case, constant monomer input does not lead to a stationary cluster size distribution, although no gelation takes place. A case where this in fact occurs is given, for instance, by the kernel $m_{1}^{-2}+m_{2}^{-2}$. In this case all concentrations decay as $t^{-1 / 3}$ irrespective of the power law $\omega$, that is, independently of the rate at which monomer is fed into the system. The systematic nature of the approach developed here is worth emphasizing: while I believe most of the results stated in this section to be well-known, I am not aware of a similarly straightforward unified derivation.

\subsubsection{Spatially inhomogeneous systems}

Let us now look at spatially inhomogeneous systems. As it frequently happens that monomer injection occurs at a well localized position, such systems are of genuine interest. At first, one might argue that they must lie outside the domain of validity of mean field theory. This is not generally the case, however: In order for the mean field approximation to hold, one requires that there be no correlations at the molecular level. Therefore, we must certainly exclude cases in which the density varies significantly on the scale of typical interaggregate distances. It often happens, though, that one has homogeneity over such scales and yet a slow spatial variation giving rise to diffusive dynamics being superimposed upon the reaction process. This is the situation we shall consider. For closely related work, see e.g [17], but the literature on this kind of problems is considerable.

We are therefore led to the following kinetic equations

$$
\begin{aligned}
\partial_{t} c(m, \vec{r})= & \frac{1}{2} \int_{0}^{\infty} d m_{1} d m_{2} K\left(m_{1}, m_{2}\right) c\left(m_{1}, \vec{r}\right) c\left(m_{2}, \vec{r}\right) \times \\
& \times\left[\delta\left(m_{1}+m_{2}-m\right)-\delta\left(m_{1}-m\right)-\delta\left(m_{2}-m\right)\right]+ \\
& +D(m) \Delta_{\vec{r}} c(m, \vec{r})+p \delta(\vec{r}) \delta\left(m-m_{0}\right)\left(t_{0}+t\right)^{\omega} .
\end{aligned}
$$

In the scaling approach we now introduce two time dependent growing quantities, namely $s(t)$ which is the typical size of aggregates, and $L(t)$, which the typical distance over which aggregate concentrations vary significantly. We 
then require as a consistency condition on our approximations, that $L(t)$ be much larger than the radius of a typical aggregate. As we have discussed in the Introduction, the relation betwen mass and radius is by no means a trivial issue and depends sensitively on the particular model. However, in a large variety of cases, the asymptotic relationship between the mass $s(t)$ and the radius $R(t)$ can be described by

$$
s(t) \approx R(t)^{D_{f}}
$$

where $D_{f}$ is the fractal dimension of the aggregate and lies between one and the space dimension $d$. The minimal requirement for the applicability of meanfield therefore becomes

$$
L(t) \gg s(t)^{1 / D_{f}}
$$

for al $t$. In particular, if the growth exponent of $L(t)$ is less than $z / D_{f}$, then mean-field will at best be applicable during a finite range of times, and nonmean-field behaviour will dominate the system at large times. In the opposite case, van Dongen has determined the limit of validity of mean-field theory by considering the size of the fluctuations and the efficiency of their transport. If one assumes that the diffusion constants $D(m)$ decay with an exponent $m^{-\gamma}$, where $\gamma>0$, it is found that the critical dimension $d_{c}$ above which mean-field holds, at least qualitatively, is given by

$$
d_{c}=\frac{1}{1-\lambda-\gamma}
$$

if the r.h.s. is positive, and is infinite otherwise.

\subsubsection{Scaling Theory for Inhomogeneous Systems}

Let us develop a scaling theory for (3.106). We limit ourselves to the case in which the injection occurs at the origin and we transform to spherical coordinates in order to exploit the existing symmetry. As definition of the scaling limit we choose

$$
\int_{0}^{\infty} d m m \int_{0}^{\infty} d r r^{d-1} c(m, r ; t) f\left(\frac{m}{s(t)}, \frac{r}{L(t)}\right) \rightarrow \int_{0}^{\infty} d x x \Phi(x, y) f(x, y)
$$

Here $s(t)$ denotes, as usual, a typical size and $L(t)$ a typical length. 
We use the same procedure as always and find for the scaling function $\Phi(x, r)$

$$
I_{1}(\rho)=I_{2}(\rho)+I_{3}(\rho)+1
$$

where the various $I_{j}(\rho)$ are defined as

$$
\begin{aligned}
I_{1}(\rho, \sigma)= & \int_{0}^{\infty} d x_{1} d x_{2} d r r^{d-1} K\left(x_{1} . x_{2}\right) x_{1} \Phi\left(x_{1} . r\right) \Phi\left(x_{2} . r\right) \times \\
& \times e^{\rho x_{1}-\sigma r}\left(1-e^{-\rho x_{2}}\right) \\
I_{2}(\rho, \sigma)= & \sigma^{2} \int_{0}^{\infty} d x d r r^{d-1} x^{1-\gamma} \Phi(x, r) e^{-\sigma r}\left(1-\frac{d-1}{\sigma r}\right) \\
I_{3}(\rho, \sigma)= & \int_{0}^{\infty} d x d r r^{d-1} x \Phi(x)[1+\omega-z \rho-\nu \sigma] e^{-\rho x-\sigma r} .
\end{aligned}
$$

Here $z$ and $\nu$ stand for the exponents with which the typical size and length grow with time respectively:

$$
s(t)=\text { const. } \cdot t^{z} \quad L(t)=\text { const. } \cdot t^{\nu}
$$

and their values are found as in the usual case during the derivation of (3.111). These are

$$
z=\frac{\omega+2}{1-\lambda} \quad \nu=\frac{1}{2}(1-\gamma z)
$$

The last relation is physically easy to inetrpret if one remarks that it is equivalent to

$$
L(t) \simeq \sqrt{D[s(t)] t}
$$

The fundamental length scale is therefore the one on which a typical cluster diffuses in time $t$.

What can one deduce from (3.111)? These are still quite formidable equations, and it is not clear how to get results out of them. The following approach yields some results: consider an arbitrary exponent $\alpha>0$ and look at the curve defined by $\sigma=\rho^{\alpha}$, where both $\rho$ and $\sigma$ go to infinity. If we further assume that

$$
\lim _{\sigma / \rho^{\alpha}=s} \Phi\left(\frac{x}{\rho}, \frac{r}{\sigma}\right)=\phi(s) \rho^{\tau(\alpha)},
$$


we find straightforwardly the following orders of magnitude at large $\rho$ and $\sigma$ for the $I_{j}(\rho)$ :

$$
\begin{aligned}
& I_{1}(\rho, \sigma)=\rho^{-\lambda-3+2 \tau(\alpha)-d \alpha} \\
& I "(\rho, \sigma)=\rho^{(2-d) \alpha-2+\gamma+\tau(\alpha)} \\
& I_{3}(\rho, \sigma)=\rho^{-2-d \alpha+\tau(\alpha)}
\end{aligned}
$$

Matching these and taking also the constant term into account one eventually finds the following relation between $\tau(\alpha)$ and $\alpha$ : first, if $\alpha>-\gamma / 2$, then we define

$$
\alpha_{c}=\frac{1-2 \gamma-\lambda}{4-d}
$$

Note that we shall always limit ourselves to the case $d<4$, since otherwise it follows from well-known results that the aggregation process becomes irrelevant and the whole system reduces to a non-interacting diffusion.

One then finds for $\tau(\alpha)$

$$
\tau(\alpha)= \begin{cases}\frac{1}{2}(d \alpha+\lambda+3) & \left(\alpha<\alpha_{c}\right) \\ 1+\lambda+2 \alpha+\gamma & \left(\alpha>\alpha_{c}\right)\end{cases}
$$

and similarly, if $\alpha<-\gamma / 2$, we find a different value of $\alpha_{c}$ :

$$
\alpha_{c}=\frac{1-\lambda}{d}
$$

and the values of $\tau(\alpha)$ are given by

$$
\tau(\alpha)= \begin{cases}\lambda+1 & \left(\alpha<\alpha_{c}\right) \\ \frac{1}{2}(d \alpha+\lambda+3) & \left(\alpha>\alpha_{c}\right)\end{cases}
$$

What do these results mean? A dependence $\tau(\alpha)$ suggests a singular behaviour at the origin of the type

$$
\phi(x, r) \simeq \exp \left[-\ln x \cdot \tau\left(\frac{\ln r}{\ln x}\right)\right] .
$$

For the sake of comparison with known results, consider the case of the constamt kernel with constant diffusion. We are therefore always in the first case, 
since $\alpha>0$. The singularity structure predicted by the theroy is therefore

$$
\Phi(x, r) \simeq x^{-3 / 2} r^{-d / 2}+x^{-1} r^{-2}
$$

and the critical line which separates the regions in which one or the other summand dominates is given by the relation $r=x^{1 /(4-d)}$. The exact connection of these results with the related ones published in [17] is not entirely clear to me, but it is certainly very close. There it is claimed that in the stationary case, the scaling variable is $m / r^{4-d}$ and that the $\tau$ exponent is given by $(6-$ $d) /(4-d)$, which is exactly the value of $\tau\left(\alpha_{c}\right)$ in our formalism.

\subsubsection{Higher Order Reactions}

Let us now consider the case in which reactions of higher order than two may also be present. The reaction term of order $r$ is given by

$$
\begin{aligned}
& \frac{1}{r !} \int d m_{1} \cdot \ldots \cdot d m_{r} K_{r}\left(m_{1}, \ldots, m_{r}\right) c\left(m_{1}, t\right) \cdot \ldots \cdot c\left(m_{r}, t\right) \times \\
& \times\left[\delta\left(m_{1}+\ldots+m_{r}-m\right)-\sum_{r^{\prime}} \delta\left(m_{r^{\prime}}-m\right)\right] .
\end{aligned}
$$

Denoting (in this subsection only) the degree of homogeneity of the kernel $K_{r}$ by $\lambda_{r}$, we obtain by a formal reasoning entirely analogous to that used in Appendix A the following conditions on the typical size $s(t)$ :

$$
\dot{s}(t)=W^{r-1} s(t)^{\lambda_{r}-r+2}
$$

At this stage, however, I must emphasize that this derivation is not rigorous for reactions of order $r$, when $r \geq 3$, for reasons explained in detail in Appendix B. Let us nevertheless assume it for the time being.

If therefore two different orders $r_{1}$ and $r_{2}$ are relevant in the same physical process, we might expect that one will invariably dominate the other in the scaling limit, unless

$$
\lambda_{r_{1}}-\lambda_{r_{2}}=r_{1}-r_{2}
$$

As we shall see later, however, the situation is more complex. The crucial issue is (3.125). As stated above and set out in detail in Appendix B, this derivation is subject to certain objections which do not arise in the two-body case. It is perfectly possible for a scaling limit to exist, with a well-defined scaling function $\Phi(x)$ satisfying some form of an equivalent scaling equation, and yet 
for (3.125) not to hold. Under such circumstances, which we will discover in certain exactly solvable models, a more complicated situation arises, which we shall not discuss in the general framework of scaling theory because of its complexity. We shall fully discuss the exactly solvable case, however, in subsection 4.8 .

Let us now look more carefully at the pure case of three-body reactions. Let us first describe the various exponents describing a three-body kernel. We define $\lambda_{3}, \mu_{1}$ and $\mu_{2}$ as follows

$$
\begin{array}{ll}
K(x, y, z)=z^{\lambda_{3}} k_{2}(x / z, y / z) & \\
k_{2}(y, z)=z^{\lambda_{2}} k_{1}(y / z) & (y, z \ll 1) \\
k_{2}(y, z)=\Gamma_{1}(y) z^{\mu} & (z \ll 1) .
\end{array}
$$

Note that, since the arguments of $K(x, y, z)$ can always be written in ascending order, it is enough to specify $k_{2}(x, y)$ on the triangle $0 \leq x \leq y \leq 1$. Conversely, $k_{2}(x, y)$ can be chosen arbitrarily on this domain, so that no limitations on the values of the above exponents hold. From this follows that (3.127) gives all the relevant information concerning the possible limiting behaviours of $K(x, y, z)$. In particular, one readily obtains the following limiting behaviour for $\Gamma_{1}(x)$ :

$$
\Gamma_{1}(x)=\text { const. } \cdot x^{\lambda_{2}-\mu} \quad(x \rightarrow 0)
$$

Note that (3.127) is not the most general behaviour imaginable: it would be possible, for example, to define $k_{2}(y, z)$ in such a way that its decay exponent $\mu$ as $z \rightarrow 0$ depended on the value of $y$. We disregard such cases and leave their treatment to the interested reader.

We now proceed quite similarly to the case of binary reactions and obtain an equation for the scaling function $\Phi(x)$ of the following form

$$
I_{3}(\rho)=\rho \int_{0}^{\infty} x^{2} \Phi(x) e^{-\rho x} d x
$$

where $I_{3}(\rho)$ is given by

$$
I_{3}(\rho)=\frac{1}{2} \int d x d y d z K_{3}(x, y, x) \Phi(x) \Phi(y) \Phi(z) x e^{-\rho x}\left[1-e^{-\rho(y+z)}\right]
$$

A fundamental difference between this case and the two-body reaction case should again be observed: in the derivation of the scaling equation for $\Phi(x)$ 
in the two-body case, we saw in appendix A that the behaviour of the small aggregates does not couple to that of the aggregates in the scaling regime: in other words, we did not need to invoke anything beyond scaling to justify the scaling equation (3.29). On the other hand, in the case of (3.130), a problem may arise from the three-body reactions involving two large particles and one small one. The technical issue involved is discussed in detail in Appendix B for the three body case. As we shall see in exactly solvable models, such cases actually occur and signal the necessity of choosing a different value for the growth exponent $z$ than the one predicted by (3.125). This is a qualitatively new phenomenon, which does not occur in the two-body case.

The large- $\rho$ behaviour can again be estimated as in appendix C. This leads to

$$
\begin{gathered}
I_{3}(\rho)=B_{1} \rho^{-\left(\lambda_{3}+4\right)} \Phi(1 / \rho)^{3}+B_{2} \rho^{-\left(\lambda_{2}+3\right)} \Phi(1 / \rho)^{2}+ \\
+B_{3} \rho^{-2-\mu} \Phi(1 / \rho) .
\end{gathered}
$$

Here $B_{i}$ are three integrals given in appendix D. One now proceeds as in the binary reaction case. The following cases arise, excepting always the possibility that a divergence at the origin occurs leading to a different value of the exponent $z$ :

(1) Case I: $\mu>0$ and $\lambda_{3}-2 \lambda_{2}<0$ : This essentially corresponds to Case I in the binary situation. One finds $\tau=1+\lambda_{3} / 2$ and the corresponding value of $1 / 2$ for the decay exponent $w$ for the small aggregates, which is the lowest possible value for $w$ in the purely ternary case. For this to be consistent we additionally need the condition $\lambda_{3}<2 \mu$. If this is violated, we have a divergence at the lower end of the integral and a different value for the exponent $z$.

(2) Case II: $\mu>0$ and $\lambda_{3}-2 \lambda_{2}>0$ : This case is new. One finds $\tau=1+\lambda_{2}$, and correspondingly

$$
w=\frac{1-\lambda_{2}}{2-\lambda_{3}}
$$

In this case, the second term in (C.4) dominates. Again, for consistency we need $\lambda_{2}<\mu$, otherwise we again have divergence at the lower end of the integral and a modified value of the exponent $z$.

(3) Case III: $\mu=0$ : This case corresponds to Case II for binary reactions. In this case, $\tau$ may take any value within a certain range: in order for there to be no divergence at the origin, we require $\tau<1$, and on the other hand, in order for the third term in (C.4) to dominate one needs

$$
\tau<\min \left(1+\lambda_{3} / 2,1+\lambda_{2}\right) .
$$

(4) Case IV: $\mu<0$ : In this case, we cannot have a finite value of $\tau$. If we have normal behaviour, then the function $\Phi(x)$ vanishes at the origin 
faster than any power law.

We shall find the above theory indeed to be violated in the following exactly solved cases:

$$
\begin{aligned}
& K_{1}\left(m_{1}, m_{2}, m_{3}\right)=m_{1}+m_{2}+m_{3} \\
& K_{2}\left(m_{1}, m_{2}, m_{3}\right)=m_{1} m_{2}+m_{2} m_{3}+m_{1} m_{3}
\end{aligned}
$$

$K_{1}$ has $\lambda_{3}$ equal to 1 and $\lambda_{2}$ and $\mu$ equal to zero, but the $z$ exponent is equal to 2 and $\tau=3 / 2^{13}$. For $K_{2}$ we have $\lambda_{3}$ equal to $2, \lambda_{2}$ equal to one and $\mu$ equal to zero. In this case we find gelation with a divergence exponent $z$ of -2 and $\tau=2.5$, that is, behaviour quite similar to the product kernel in the binary case. These results will be shown in detail in subsection 4.8 .

\subsubsection{Multicomponent Aggregation}

An interesting generalization of the aggregation model consists in allowing the mass parameter $m$ to become a vector $[69,51]$. The meaning of the components could be, for instance, the concentration of various species present in the aggregates. Similarly, we can think of one species as being charged and the other neutral, so that the reactivity of an aggregate depends not only on its total mass, but on the total number of monomers of the charged species. The reaction rates $K\left(\vec{m}_{1}, \vec{m}_{2}\right)$ then depend on two additive parameters and are therefore an instance of the possibility of multicomponent aggregation.

Apart from its relevance in realistic aplications (for an interesting use of related concepts concerning the coagulation of charged aggregates, see for example [39]), the real interest in studying this case comes from the possibility of looking at a different kind of scaling behaviour: Indeed, so far, we have looked only at the average size of the aggregates. Here, as I shall show shortly, under quite general circumstances the limiting distribution consists of aggregates which have a fixed composition determined by the masses of the various species initially present. A natural question then follows: on what scale does the distribution of compositions vary? It is less than the typical size, so we obtain a scaling in which two different sizes play an important role. In fact, we show that this distribution is quite generally a Lévy stable distribution ${ }^{14}$. Further, it can be shown that the index of the Lévy distribution is given by the ratio of the exponents characterizing the growth of composition fluctuations to the exponent $z$ for the growth of the typical cluster size. These results are in fact to be expected: indeed, we may consider the following stochastic model

\footnotetext{
${ }^{13}$ As if we were in fact dealing with Case I. Is this more than a coincidence? I make no claim to know

${ }^{14}$ possibly an ordinary Gaussian.
} 
for aggregation. Take a finite but large set of aggregates of various compositions. Then choose two at random according to the rates $K\left(\vec{m}_{1}, \vec{m}_{2}\right)$ and join them. Let this process be repeated indefinitely. It is then quite likely that an appropriate generalization of the law of large numbers will apply, so that all aggregates tend to a fixed composition. If one then wishes to analyze further on what scale the composition varies, one needs some version of the central limit theorem. (Similar random coagulation processes have been looked into in [82], but not, it seems to me, to the extent of proving such claims.) Such a theorem would state that the resulting distribution is always Lévy and that the typical size of the fluctuations is connected to the index of the Lévy law involved [8]. Note that the constant kernel corrresponding to such a case was already studied in $[69,51]$. Defining

$$
|\vec{m}|=\sum_{k} m_{k}
$$

the scaling assumption now reads

$$
\lim _{t \rightarrow \infty} \int_{0}^{\infty} d \vec{m}|\vec{m}| c(\vec{m}, t) f\left(\frac{\vec{m}}{s(t)}\right)=\int_{0}^{\infty} d \vec{x}|\vec{x}| \Phi(\vec{x}) f(\vec{x})
$$

Using exactly the same techniques as in the one component case, one derives the following equivalent of the scaling equation (3.29):

$$
\begin{array}{r}
\int d \vec{x} \vec{x} \cdot \vec{\nabla} f(\vec{x}) \vec{x}=\int d \vec{x}_{1} d \vec{x}_{2} K\left(\vec{x}_{1}, \vec{x}_{2}\right) \Phi\left(\vec{x}_{1}\right) \Phi\left(\vec{x}_{2}\right) \times \\
\times\left[f\left(\vec{x}_{1}+\vec{x}_{2}\right)-f\left(\vec{x}_{1}\right)\right]
\end{array}
$$

In order to simplify notation, I limit myself to the case of two components, though the general case presents no difficulties of principle. A moment's thought shows that the following is a solution of (3.138):

$$
\Phi(\vec{x})=\Phi_{1}\left(x_{1}\right) \delta\left(x_{1}-x_{2}\right)
$$

where $x_{i}$ denotes the $i$-th component of $\vec{x}$ and $\Phi_{1}(x)$ is the scaling function corresponding to the rates $K\left(x_{1}, x_{1} ; x_{2}, x_{2}\right)$. There exist further solutions differing from (3.139) by trivial normalizations, but since we can always fix units so as to have the same mass of both components initially present, this is irrelevant. I do not know whether further solutions exist, but the plausible considerations made at the beginning of this subsection concerning the law of large numbers appear to speak against such a possibility.

Let us now look at the variations in composition and the way in which they scale. To fix ideas, we start from a system in which the mass initially contained 
in both components is the same, and we assume that the scaling function given in (3.139) is indeed the relevant one. This permits us to neglect the dependence of the reaction rates on composition, since a fixed composition is reached at the scale defined buy the typical size $s(t)$. . We then define a scaling function $\Psi(\sigma, \delta)$ for the sum and difference between $x_{1}$ and $x_{2}$. We define it as follows:

$$
\begin{aligned}
\lim _{t \rightarrow \infty} & \int_{0}^{\infty} d m_{1} d m_{2}\left(m_{1}-m_{2}\right) c(\vec{m}, t) f\left(\frac{m_{1}+m_{2}}{s_{1}(t)}, \frac{m_{1}-m_{2}}{s_{2}(t)}\right)= \\
& \int_{0}^{\infty} d \sigma \int_{-\infty}^{\infty} d \delta \delta \Psi(\sigma, \delta) f(\sigma, \delta) .
\end{aligned}
$$

Here $s_{1}(t)$ corresponds to the typical size of aggregates, whereas $s_{2}(t)$ gives the scale for the variations in composition. In Appendix E. we derive a scaling equation (E.1) for $\Psi(\sigma, \delta)$ and show that

$$
\Psi(\sigma, \delta)=\sigma^{-\alpha} \Phi_{1}(\sigma) \chi_{\alpha}\left(\frac{\delta}{\sigma^{\alpha}}\right)
$$

is a solution. Here $\Phi_{1}(x)$ is the one-component scaling function of (3.139), $\chi_{\alpha}(x)$ is the symmetric Lévy stable distribution of index $1 / \alpha$ given by the Fourier transform of $\exp \left(-|q|^{1 / \alpha}\right)$ with respect to $q$ and $\alpha$ is given by

$$
\alpha=\lim _{t \rightarrow \infty} \frac{\dot{s}_{2}(t) s_{1}(t)}{\dot{s}_{1}(t) s_{2}(t)}
$$

In order to determine $\alpha$ unambiguously, we argue as follows: All Lévy distributions except for the Gaussian have power-law tails. Further, if $\alpha \geq 1$, it is known that the first moment of the distribution diverges. This can certainly not happen if, as we have always supposed, we start from initial conditions having finite mass. It is also known that the $\chi_{\alpha}$ are only positive if $\alpha \geq 1 / 2$. We therefore have $1 / 2 \leq \alpha<1$. The value $1 / 2$ corresponds to a gaussian distribution, whereas all other allowable values correspond to a power-law decay of $x^{-1-1 / \alpha}$. Thus a value of $\alpha$ different from $1 / 2$ implies that the differences in composition have power-law tails extending to infinity in the large time limit. Since no mechanisms are known to produce these unless they be present in the initial conditions, we may state that if the initial conditions do not have powerlaw tails, the final distribution must be Gaussian ${ }^{15}$. If the initial distribution did contain a power-law tail, then it is quite likely that the corresponding Lévy stable law will be the relevant one in the scaling regime. We therefore

\footnotetext{
$\overline{15}$ This is maybe a little less obvious than it seems: as we shall see later in detail, the large $x$ asymptotics in the scaling regime has no simple relationship to the large $m$ asymptotics at fixed times. It is, however, a very reasonable assumption.
} 
see that initial conditions are relevant in this case to determine the asymptotic behaviour of the typical size of the variations in the aggregate composition. We shall later find similar behaviour in ballistic aggregation, which is, in fact, a closely related problem.

\subsection{Non-scaling Asymptotics}

The above is a subject which, from the peculiarly biased viewpoint I have been taking in this paper, will naturally receive rather short shrift. Nevertheless, it is rather obvious that the following questions are relevant:

(1) How does $c(m, t)$ behave at large times for fixed $m$ ?

(2) Conversely, how does $c(m, t)$ behave for large $m$ at fixed $t$ ? Obviously, the decay should, in some sense be exponential, or at least fast enough, but more detailed information concerning power-law corrections to the exponential decay is also important.

(3) What is the intermediate asymptotics of the $c(m, t)$ ? By this I mean the following: frequently, there exists a time dependence $f(t)$ such that

$$
c(m, t)=g(m) f(t)[1+o(1)] \quad(t \rightarrow \infty)
$$

for fixed $m$. In this case, one may ask what is the large $m$ behaviour of $g(m)$. In the absence of such a function $f(t)$, the whole issue becomes rather ambiguous.

(4) Similarly, the problem of the large time behaviour of the moments of the cluster-size distribution is of considerable physical relevance, since these are usually its most easily accessible characteristics. The problem splits into two disjoint issues.

First, the large time behaviour of moments $M_{p}(t)$ of order $p$ such that

$$
\int_{0}^{\infty} x^{p} \Phi(x)=\infty,
$$

falls outside the purview of standard scaling theory. The problem, of course. lies in the fact that these low-order moments are dominated by the low- $m$ part of the cluster-size distribution, whereas the higher-order moments are determined by the distribution as a whole.

On the other hand, high-order moments may also cause difficulties, though perhaps of a more trivial kind: indeed, if the initial condition contains a power-law contribution, then some sufficiently high-order moment will diverge from the very beginning., which is a clear sign that we may not blindly apply the scaling approach in evaluating moments. This is seen, for example, in the exactly solved model of the constant kernel, 
for which I show that scaling is approached whenever the initial distribution has finite first moment. Nevertheless, it is obvious that the second moment has no well-defined large-time asymptotics if its initial value is divergent. One therefore sees that stronger assumptions than merely the existence of the scaling limit are required in order to justify the usual scaling expressions even for high-order moments.

In the literature, such issues have often been (mistakenly) discussed as socalled "violations of scaling" (see e.g. [63,64] for a characteristic example of such confusion. The issue of scaling in parity dependent kernels will be taken up again later in this paper.) In the following, we shall always refer to our standard definition of scaling, and will only consider as non-scaling such systems as do not approach a limiting form in the sense defined by (3.12). In fact, as we shall see, all of the quantities described above may exhibit unexpected behaviour while the scaling hypothesis remains fulfilled. We shall, in our terminology, call such deviations from expected behaviour, violations of strong scaling. Since I have never been too explicit about what is meant by strong scaling, it is clear enough that anything peculiar enough to attract some attention might be brought under this heading. In the following, I shall largely follow the approach sketched in $[62]^{16}$.

Let us first turn to the large time behaviour of $c(m, t)$ at fixed $m$. In this case, production of clusters of mass $m$ can be neglected, and I may approximate (2.2) by

$$
\dot{c}(m, t)=-c(m, t) \int_{0}^{\infty} K\left(m, m^{\prime}\right) c\left(m^{\prime}, t\right) d m^{\prime}
$$

Using the definitions of $\mu$ and $\nu$ given in (3.4) and (3.5) as well as the fact that the relevant masses $m^{\prime}$ in (3.145) are much larger than $m$, one eventually obtains the following approximate differential equation for $c(m, t)$

$$
\dot{c}(m, t)=\text { const. } \cdot m^{\mu} M_{\nu}(t) .
$$

In order to understand the behaviour of this system, we must first know the behaviour of $M_{\nu}(t)$. Assume strong scaling, after which one may check for self-consistency. In this case, one finds for kernels of type III

$$
M_{\nu}(t)=\text { const. } \cdot t^{(\nu-1) /(1-\lambda)},
$$

\footnotetext{
16 The reader should be warned that this paper thoroughly confuses the two concepts of scaling regime and non-scaling asymptotics.
} 
from which follows that $c(m, t)$ decays as a stretched exponential of the form

$$
c(m, t)=\text { const. } \cdot \exp \left[- \text { const. } \cdot m^{\mu} t^{-\mu /(1-\lambda)}\right]
$$

Note that this is in perfect agreement with the predictions of strong scaling, so that no violations are to be expected in Case III. Of course, this might not be viewed as quite conclusive, since we need a hypothesis to determine the large-time behaviour of $M_{\nu}(t)$. As we shall see, however, the main hypothesis is that the expression of the moment as an integral of the scaling function should have no singularities at the origin, which is trivially satisfied in Case III. Another source of problems might arise from power.law initial conditions, but again, as long as $\nu<1$ no divergence is possible.

In case I, the above approach fails, since it is readily seen that $M_{\nu}(t)$ cannot be described by a convergent integral of the scaling function. The following observation, on the other hand, yields interesting results [67]: if one introduces the following new variables in the discrete representation, which turns out here to be more convenient,

$$
\begin{aligned}
\phi_{j}(\theta) & =c_{j}(t) / c_{1}(t) \\
d \theta & =c_{1}(t) d t,
\end{aligned}
$$

the original equations (2.2) become

$$
\frac{d \phi_{j}}{d \theta}=\frac{1}{2} \sum_{k=1}^{j-1} K(k, j-k) \phi_{k} \phi_{j-k}-\phi_{j} \sum_{k=1}^{\infty}[K(k, l)-K(1, l)] \phi_{l} .
$$

These equations have a non-trivial equilibrium solution $a_{j}$ which can sometimes be determined recursively. It can be worked out for kernels of the form $K(j, k)=(j k)^{\lambda / 2}$,(see [61]), as well as more generally [103] that these constants behave as $j^{-\tau}$ with $\tau=1+\lambda$, which is therefore in good agreement with the qualitative behaviour predicted by strong scaling. This also settles the issue of intermediate asymptotics as defined above, showing that it indeed exists, and that it coincides with the asymptotic behaviour of small clusters in the scaling limit.

One further finds using (2.2) that $c_{1}(t)$ goes as $t^{-1}$, from which follows that all $c_{j}(t)$ do. This demonstration has been put on a firmer footing by a rigorous inductive proof by van Dongen and Ernst [103]. On the other hand, there remain discrepancies between the behaviour of the $a_{j}$ at large $j$ and that of the scaling function $\Phi(x)$ at small $x$ : in particular, at least under the assumption of regular behaviour for $\Phi(x)$, the prefactors of both differ. 
Finally, for case II, no such arguments are available. In particular, neither an approach based on (3.146) nor one based on recursion relations can be justified. Further, a large number of counterexamples are known, which precisely belong to this case. In fact, both non-gelling exactly solved classical models, the constant and the sum kernels, belong to case II, as also do some variations on the constant kernel for which the strangest deviations from strong scaling have been reported.

It remains to discuss shortly the issue of the large $m$ behaviour at fixed time [25]. In this case one may safely neglect the reaction of particles of mass $m$ with larger aggregates, so that we have the following approximate equations, for the discrete case:

$$
\dot{c}_{j}=\frac{1}{2} \sum_{k=1}^{j-1} K(k, j-k) c_{k} c_{j-k}
$$

Again, for monodisperse initial conditions, this can be solved in a straightforward way by recursion, using the ansatz:

$$
c_{j}(t)=a_{j} t^{j-1}
$$

One finds (for details see [25]) that $a_{j}$ goes as $j^{-\lambda}$ for all kernels $K(k, l)$ with $\nu<1$. A comparison with the results obtained for the large $x$ end of $\Phi(x)$ shows that a difference between the two can, and in fact does occur for the case $\nu=1$ [106]. It therefore follows from these results that the large mass end of the cluster size distribution at fixed times always decays exponentially, a fact which we shall find to be of some importance in assesssing the possibility to describe certain models by mean-field equations.

Let us finally consider the scaling prediction for the moments. Overall, the strong scaling prediction for the asymptotic behaviour of $M_{p}(t)$ is

$$
M_{p}(t)=\bar{M}_{p} s(t)^{p-1} \quad(t \rightarrow \infty)
$$

The immediate case in which this cannot hold is, of course, for values of $p$ such that $\bar{M}_{p}$ does not exist (diverges). Clearly, this can only occur when $\Phi(x)$ has a power-law singularity at the origin, since the exponential decay of $\Phi(x)$ at infinity shown in subsection 3.5.2 precludes any divergence there. Thus there are no problems in Case III, but whenever $\tau$ is finite, the asymptotic behaviour (3.153) is invalid in the range

$$
p \leq-1+\tau
$$


However, as we shall see later in exactly solved examples, this behaviour can also fail outside the range stated in (3.154). Indeed, in various kernels of type II one finds that for large $t$ at fixed $m$

$$
c(m, t)=t^{-w^{\prime}(m)}[1+o(1)] \quad(t \rightarrow \infty) .
$$

Here $w^{\prime}(m)$ varies continuously with $m$ and bears no necessary relation to either $z$ or $\tau$. In this case, it may well happen that $(p-1) z$ be less than $\max _{m} w^{\prime}(m)$, so that the decay of $M_{p}(t)$ is dominated by that of a specific type of aggregate. We shall in fact see such examples in the next section. This definitely cannot happen, however, for moments which grow in time, that is whenever $p>1$.

An altogether different violation of the behaviour stated in (3.153) occurs whenever the initial conditions have a power-law tail. In this case, as one sees in many exactly solved cases, the large mass behaviour always maintains this tail, which cannot be eliminated by any aggregation process except gelation. In this case, clearly, whenever $p$ is large enough to cause the divergence of $M_{p}(0)$, the moment $M_{p}(t)$ is identically infinite, so that (3.153) becomes meaningless. Nevertheless, scaling often holds also under these conditions. In such cases, we must reinterpret (3.153) to mean

$$
\int_{0}^{\Lambda s(t)} m^{p} c(m, t) d m=[s(t)]^{p-1} \int_{0}^{\Lambda} x^{p} \Phi(x) .
$$

where $\Lambda$ is an arbitrary constant. The time necessary for (3.156) to become valid goes to infinity as $\Lambda$ does.

\subsection{The Moment Equations}

The moments $M_{p}(t)$ of the cluster size distribution function satisfy some gen-

eral exact equations, which are in many cases of considerable use, though we shall not make much explicit use of them in this paper. Indeed, from (2.2) follows immediately

$$
\begin{gathered}
\frac{d M_{p}}{d t}=\int_{0}^{\infty} d m_{1} d m_{2} K\left(m_{1}, m_{2}\right) c\left(m_{1}, t\right) c\left(m_{2}, t\right) \times \\
\times\left[\left(m_{1}+m_{2}\right)^{p}-m_{1}^{p}-m_{2}^{p}\right]
\end{gathered}
$$


For integer values of $p$, these take a particularly striking form:

$$
\frac{d M_{p}}{d t}=\sum_{k=1}^{p-1}\left(\begin{array}{l}
p \\
k
\end{array}\right) \int_{0}^{\infty} d m_{1} d m_{2} m_{1}^{k} m_{2}^{p-k} K\left(m_{1}, m_{2}\right) c\left(m_{1}, t\right) c\left(m_{2}, t\right) .
$$

The two principal uses of these relations are the following: first, in the case of the bilinear kernel (4.68), they yield closed equations, which can be solved recursively. Their solution for low values of $p$ is one of the quickest ways to obtain qualitative information concerning the solution. In particular, it readily yields the exponent $z$ as well the gel time in gelling systems.

On the other hand, they can also be used for estimating the solution from above or below. Thus White's proof of global existence for the solutions of (2.2), see [112], rests essentially on such an estimate which follows from the estimate

$$
K\left(m, m^{\prime}\right) \leq C\left(m+m^{\prime}\right)
$$

The possibility of such estimates is a result of the positivity of the right-hand side of (3.158). One then obtains a closed set of differential inequalities, which can then, because of their recursive character, be solved to yield upper bounds on the $c(m, t)$. Similarly, we used inequalities of the same nature in subsection 3.5.2 to estimate the high moments of the scaling function $\Phi(x)$. Note carefully that the moment equations (3.158) are not at all equivalent to the corresponding equations (3.36) for the moments of $\Phi(x)$ : the latter only describe the moments if the system approaches scaling, whereas the former hold in every case. In fact, the knowledge of all moments is equivalent, in principle, to knowing the full cluster size distribution, so that the solution of (3.158) is in principle of the same degree of difficulty as the full solution of the original Smoluchowski equations (2.2). The solution of (3.158) therefore also contains indications of all corrections to scaling and non-scaling asymptotics, which, as we have seen, often differ considerably from the scaling behaviour. These two equations cannot, therefore, be identified. Nevertheless, it is certainly true that the leading behaviour of $M_{p}(t)$ for $p$ sufficiently large only depends on the scaling function $\Phi(x)$. It might therefore be possible to prove the scaling hypothesis by a careful study of the leading terms for the large-time behaviour of (3.158). 


\section{Exactly Solved Models}

In the following, I shall discuss a large variety of exactly solved models. One can justify interest in such cases in several ways: they are in themselves a source of pleasure, they are an ideal test ground for numerical work and they allow us better to understand the structure of solutions in general. While I sympathize to some extent with all of these motives, in this paper I shall be primarily concerned, as I have been throughout, with the issue of the validity of scaling theory. Exact models are, of course, invaluable in this respect, since we have ordinarily a complete overview of the solutions behaviour. It is therefore straightforward to say in which way the system does ineed satisfy the scaling hypothesis, and to what extent it may occasionally deviate from what is expected. As we shall see, the scaling hypothesis as I have defined it, see (3.12), holds essentially for all systems we shall have occasion to look at ${ }^{17}$. On the other hand, as I shall show in an extensive set of examples, the set of assumptions I have denoted by the general name of "strong scaling", are in general not fulfilled. To be more accurate, it is frequently possible to find more or less natural exactly solved cases for which these assumptions fail in several ways. The detailed illustration of such failure I consider to be instructive, since it shows with great detail exactly how much we may, and more importantly what we may not, deduce from the existence of a scaling limit.

The exactly solved kernels fall in two categories. First, one might mention the so-called "classical" kernels

$$
\begin{aligned}
& K_{1}\left(m, m^{\prime}\right)=1 \\
& K_{2}\left(m, m^{\prime}\right)=m+m^{\prime} \\
& K_{3}\left(m, m^{\prime}\right)=m m^{\prime},
\end{aligned}
$$

which are known as the constant, sum and product kernels respectively. Their solution can be generalized to the case of an arbitrary linear combination of the three, see (4.68), which is known as the general bilinear kernel. The three kernels described in (4.1-4.3) are all quite different from each other, as should be expected from the general scaling theory exposed in the previous section: the first has $\lambda=0$, the second has $\lambda=1$ and the third has $\lambda=2$ and is accordingly a gelling kernel. In the first two cases, on the other hand, we have regular growth, proportional to time in the first case, exponentially fast in the second. All these predictions are indeed borne out by the exact solutions.

\footnotetext{
${ }^{17}$ The solitary exception I have in mind is the crossover between the sum kernel and the product kernel, for which I have not been able to find a scaling description. However, this may still be a soluble problem. In any case, this only concerns a crossover phenomenon, for true scaling behaviour I am not aware of any counterexamples
} 
As for the scaling theory, these kernels in general show a reasonable behaviour, that is, they usually satisfy scaling in an extremely strong form. However, even in these cases, it can be shown that many issues depend rather sensitively on initial conditions. We shall see, in particular, that initial conditions with power-law tails at large masses can significantly modify the non-scaling behaviour, even when the scaling function is unaffected.

I shall also discuss some natural extensions of these kernels to ternary reactions. This will show the way in which the scaling approach may actually break down altogether if collisions involving particles outside the scaling regime dominate the physics.

Further, since not only the three kernels above can be solved, but also the general bilinear kernel (4.68), we can clearly study various types of crossover. In fact, as I shall show, two of the three possibilities can be analyzed rather extensively and one arrives eventually at a crossover function as defined in subsection 3.6.

Finally, there are non-classical kernels, such as the $q$-sum or the parity-dependent kernels. These are characterized by the fact that they are not exactly homogeneous. As we shall see, they display a considerable variety of unexpected behaviours in their non-scaling asymptotics, but all satisfy scaling in the sense specified in this paper. Furthermore, as we shall see, even these satisfy the Dominant Singularity Hypothesis, that is, the value for the small-size exponent $\tau$ is the same as that of the large size exponent $\vartheta$. Nevertheless, the array of non-scaling anomalies shown in these cases is instructive, as it shows us that certain features one takes for granted are, in fact, peculiar features of the simpler exactly solved kernel and do not generalize to more general cases.

\subsection{The Constant Kernel}

The constant kernel defined by

$$
K\left(m_{1}, m_{2}\right)=1
$$

was first solved by von Smoluchowski [89] and its solution has been often rederived. The approach we follow is an elementary, yet instructive, use of the generating function approach: define

$$
F(\zeta, t)=\int_{0}^{\infty} c\left(m^{\prime}, t\right) e^{\zeta m^{\prime}} d m^{\prime}
$$


Here the integral must be replaced by a sum whenever $c(m, t)$ is a sum of delta functions. The fundamental equation (2.2) now takes the form

$$
F_{t}=\frac{1}{2} F(\zeta, t)^{2}-F(\zeta, t) F(0, t)
$$

After making the replacement

$$
G(\zeta, t)=F(\zeta, t)-F(0, t)
$$

(4.5) becomes

$$
\begin{aligned}
& G_{t}=\frac{1}{2} G^{2} \\
& G(\zeta, t=0)=g(\zeta):=\int_{0}^{\infty} c\left(m^{\prime}, 0\right)\left(e^{\zeta m^{\prime}}-1\right) d m^{\prime} .
\end{aligned}
$$

From this one readily obtains the following explicit form for $G(\zeta, t)$ in terms of $g(\zeta)$, which in turn is explicitly determined by the initial concentrations. The connection between the actual concentrations at time $t$ and the initial values is, as in many other cases, quite opaque. We shall see, however, that for many relevant issues the specific nature of this connection is not needed. This expression for $G(\zeta, t)$ is

$$
G(\zeta, t)=\frac{2 g(\zeta)}{2-t g(\zeta)}
$$

If, using the notations introduced in (2.3) and (2.4), I wish to compute $c_{j}(t)$ for monodisperse initial conditions, it is a straightforward excercise in power series manipulation to show that

$$
c_{j}(t)=\frac{4}{(t+2)^{2}}\left(\frac{t}{t+2}\right)^{j-1} .
$$

This is readily seen to approach a scaling form: if we namely set $s(t)=t$, we obtain using the definition (3.12) that (4.10) yields as scaling function

$$
\Phi(x)=4 e^{-2 x}
$$

It therefore follows that $\tau=0, z=1$ and $w=2$. This manifestly satisfies the scaling relation (3.25) derived in [110]. To tidy up various details, we point out that (4.11) is indeed a solution of (3.34): all functions of the form $a e^{-x / 2}$ 
satisfy it for $K(x, y)=1$ and only (4.11) satisfies the normalization condition (3.38). Furthermore, setting $s(t)$ equal to $t$, as we did, corresponds because of (A.5) to setting $W$ equal to one. Everything therefore confirms the scaling calculations down to the smallest detail. Note that, in the following, we shall usually not verify the normalization issues in great detail. Rather, we shall usually implicitly assume $W=1$, which involves a certain choice of time scale such that $s(t)$ satisfies excatly $\dot{s}=s^{\lambda}$, with no further prefactors.

Now we still need to show that this scaling form holds for arbitrary initial conditions. In itself, this is non-trivial: estimating the asymptotic behaviour of the formal expressions for $c(m, t)$ for arbitrary initial conditions is quite cumbersome, even in this most elementary case. We therefore rely on an observation made at the end of subsection 3.2: namely, in order to show scaling, it suffices to show convergence as defined by (3.14). In our case, it amounts to showing that

$$
\left.\lim _{t \rightarrow \infty} G_{\zeta}(\zeta, t)\right|_{z=s / t}
$$

exists. But this is quite straightforward using (4.9), if we may assume that $g(\zeta)$ has finite first derivative at $\zeta=0$, or, what amounts to the same, that the $c(m, 0)$ have finite first moment. Under this hypothesis, one finds that the limit defined in (4.12) is given by

$$
\frac{4}{(2-s)^{2}}
$$

which is, according to (3.14), the Laplace transform of $x \Phi(x)$ evaluated at $-s$.

This system provides various interesting examples for several of the phenomena discussed in Section 3: Let us, for example, consider initial conditions for which $c_{j}(0)$ decays as $B j^{-\alpha}$, with $2<\alpha<3$. In this case, the total mass is finite, but none of the higher integer moments converge. $G_{\zeta}(\zeta, t)$ has therefore two singularities in $\zeta$ at large times $t$ : one at, and the other near, the origin. This last is the one giving rise to the scaling contribution, since we have just shown that convergence to a scaling form does take place under these circumstances. On the other hand, the second and higher moments do not exist, due to the presence of the former singularity. It is therefore rather misleading to say that $c(m, t)$ is "well approximated" by $t^{-2} e^{-j / t}$. A more correct statement is that at large times, one has

$$
c_{j}(t) \simeq t^{-\alpha} \Phi_{1}(j / t)+4 t^{-2} e^{-j /(2 t)},
$$


where $\Phi_{1}(x)$ is a function which can, in principle be computed explicitly. In fact, one has, using a similar approach as in the derivation of (3.14):

$$
\mathcal{L}\left[x \phi_{1}(x)\right]=\frac{4 C \Gamma(1-\alpha) s^{\alpha-2}[2-2 \alpha+(3-\alpha) s]}{(2+s)^{3}}
$$

where $\mathcal{L}$ denotes the Laplace transform. It behaves as $x^{2-\alpha}$ as $x \rightarrow 0$, but becomes asymptotically equal to $x^{-\alpha}$ as $x \rightarrow \infty$. Since $\alpha>2$, it follows that the second term of(4.14) always dominates when $j / t$ is kept fixed and $t \rightarrow \infty$. On the other hand, the first term eventually dominates as $j \rightarrow \infty$ for fixed $t$. Choosing an appropriate definition of $\tau^{\prime}$ and $w^{\prime}$, however, one finds that $\tau^{\prime}=\tau$ and $w^{\prime}=w$, so that strong scaling still holds in this particular case.

Quite generally speaking, one should not confuse the fact that the distribution approaches a scaling limit, which is a global property of the full distribution at large times, with good approximations for individual values of $c_{j}(t)$. The exact solution for monodisperse initial conditions could easily lead to wrong impressions on that subject: indeed, for (4.10), one has

$$
c_{j}(t)=t^{-2} \exp (j / t)\left[1+O\left(j t^{-2}\right)+O\left(t^{-1}\right)\right]
$$

for all $j$ and $t$. This does not generalize to arbitrary initial conditions, however. In particular, for fixed values of $j$ the constant

$$
c_{j}^{(\infty)}=\lim _{t \rightarrow \infty}\left[t^{2} c_{j}(t)\right]
$$

is in general not equal to 4 , and depends on the initial data, even when these decay exponentially, as is readily verified in specific instances [108]. If the initial condition does decay exponentially, however, then $c_{j}^{(\infty)}$ also approaches 4 exponentially fast [65], as a straightforward estimate of the contour integrals yielding $c_{j}(t)$ from $(4.9)$ will show.

\subsection{The Sum Kernel}

The sum kernel is given by

$$
K\left(m, m^{\prime}\right)=m+m^{\prime}
$$

It has been solved exactly first by $[79,87]$, see also [23] for further references on the subject. I will show two approaches, which can both be used to obtain 
the full solution: the first involves a transformation, introduced by Lushnikov [67], which can always be performed when the kernel is of the form

$$
K\left(m, m^{\prime}\right)=f(m)+f\left(m^{\prime}\right)
$$

where $f$ is an arbitrary function. On the other hand, the usual approach using generating functions works quite well also. The reason for showing both approaches is, as stated in the Introduction, to give a broad overview of the different ways to attack a given problem.

Let us therefore first consider the discrete case with monodisperse initial conditions. We may then define, for arbitrary kernels of the form (4.19),

$$
\phi_{j}(\theta)=\frac{c_{j}(t)}{\sum_{k=1}^{\infty} c_{k}(t)} \quad d \theta=d t \sum_{k=1}^{\infty} c_{k}(t),
$$

where the $c_{j}(t)$ are defined from the $c(m, t)$ via (2.4). One then sees that $(2.2)$ becomes

$$
\frac{d \phi_{j}}{d \theta}=\sum_{k=1}^{j-1} f(k) \phi_{k} \phi_{j-k}-f(j) \phi_{j} .
$$

This set of equations is recursive and can sometimes be solved explicitly. In the case at hand, when $f(j)=j$, the additional substitution

$$
\psi_{j}=\phi_{j} e^{j \theta}
$$

does the job, leading to the system of equations

$$
\begin{aligned}
\frac{d \psi_{j}}{d \theta} & =\sum_{k=1}^{j-1} k \psi_{k} \psi_{j-k} \\
\psi_{j}(0) & =\delta_{j, 1}
\end{aligned}
$$

These are solved via the ansatz:

$$
\psi_{j}(\theta)=a_{j} \theta^{j-1}
$$

The coefficients $a_{j}$ are now determined by the following recurrence

$$
\begin{aligned}
(j-1) a_{j} & =\sum_{k=1}^{j-1} k a_{k} a_{j-k} \\
a_{1} & =1 .
\end{aligned}
$$


This recursion is readily solved, for example using generating functions, see Appendix Q, and yields finally

$$
a_{j}=\frac{j^{j-2}}{(j-1) !} .
$$

The sum over all concentrations obeys a closed equation (if we assume that mass is conserved for all times, which will turn out to be justified here). This leads to

$$
\sum_{k=1}^{\infty} c_{k}(t)=S_{0} e^{-t} \quad S_{0}=\sum_{k=1}^{\infty} c_{k}(0) .
$$

Note that now $S_{0}=1$. Combining all this eventually yields for the concentrations $c_{j}(t)$

$$
c_{j}(t)=\frac{j^{j-2}}{(j-1) !}\left(1-e^{-t}\right)^{j-1} \exp \left[-j\left(1-e^{-t}\right)\right] e^{-t}
$$

Note here an amusing connection: if one considers (4.21) for $f(j)=j$ and make the substitution $x_{j}=\phi_{j} / j$, one obtains

$$
x_{j}=\frac{1}{2} \sum_{k=1}^{j-1} k(j-k) x_{k} x_{j-k}-j x_{j}
$$

the $x_{j}$ therefore satisfy the Smoluchowski equations $(2.2)$ for the kernel $K(k, l)=$ $k l$, at least as long as the total mass $M_{1}(t)$ is conserved, that is, before the gelation time. This is particularly useful to evaluate the exponent $\tau$, which does not depend on the time scale. A generalization of this remark was shown in [118].

One finds that the appropriate scaling variable is $j e^{-2 t}$ and the scaling function is given by

$$
\Phi(x)=\frac{x^{-3 / 2} e^{-x / 2}}{\sqrt{2 \pi}} .
$$

To show that scaling occurs for all initial conditions, however, it is easier to use the generating function approach without further transformations. The equations for $c(m, t)$ can be cast in the form

$$
\partial_{t} c(m, t)=\int_{0}^{m} d m^{\prime} m^{\prime} c\left(m^{\prime}, t\right) c\left(m-m^{\prime}, t\right) d m^{\prime}-
$$




$$
-S_{0} e^{-t} m c(m, t)-c(m, t)
$$

where both mass conservation and (4.28) have been used to simplify the equations. If we define $F(\zeta, t)$ as

$$
F(\zeta, t)=\int_{0}^{\infty} c(m, t) e^{\zeta m} d m
$$

one obtains using (4.32):

$$
\begin{aligned}
& \partial_{t} F=\left(F-S_{0} e^{-t}\right) \partial_{\zeta} F-F \\
& F(\zeta, t=0)=f(\zeta)
\end{aligned}
$$

This can be solved by the method of characteristics, with the following result, shown in appendix $\mathrm{G}$

$$
\begin{aligned}
& F(\zeta, t)=f\left(\zeta_{0}\right) e^{-t} \\
& \zeta=\zeta_{0}-\left[f\left(\zeta_{0}\right)-f(0)\right]\left(1-e^{-t}\right)
\end{aligned}
$$

From this it follows after some algebra, that, if we assume that the initial cluster size distribution $c(m, t)$ has finite second moment, then

$$
\left.\lim _{t \rightarrow \infty}\left[\partial_{\zeta} F(\zeta, t)\right]\right|_{\zeta=s e^{-2 t}}=\frac{1}{\sqrt{1-2 f^{\prime \prime}(0) s}} .
$$

which gives for the scaling function $\Phi(x)$ :

$$
\Phi(x)=\frac{x^{-3 / 2} e^{-x /\left[2 M_{2}(0)\right]}}{\sqrt{2 \pi M_{2}(0)}},
$$

where we have used the fact that the second derivative of $f(\zeta)$ is the second moment of the initial distribution. It is certainly both remarkable and somewhat anomalous that the scaling function depends (albeit in a somewhat trivial fashion) on the initial condition.

This dependence on the initial condition, as well as the appearance of the second derivative of the generating function in the expression for the solution make it of particular importance to study the effect of highly polydisperse initial conditions, that is, such initial conditions as have $c(m, 0)$ behaving as $C m^{-\alpha}$ as $m \rightarrow \infty$, where $2<\alpha<3$, as we saw in the case of the constant kernel. To avoid tedious repetitions, we use the remark made in (4.30) in order 
to map this problem onto the corresponding problem for the product kernel, which will be treated in Appendix L. There we shall find that the typical size does not grow as $e^{2 t}$ any more, but rather as $\exp [(\alpha-1) t /(\alpha-2)]$. This is the first sign of non-universal behaviour in this system. One also finds

$$
\tau=\frac{\alpha}{\alpha-1}
$$

Note how, for $\alpha=3$, the traditional value $3 / 2$ is recovered. For $\alpha>3$ the above computations cease to be valid. On the other hand, for $\alpha \rightarrow 2$, one sees that $\tau$ approaches the limiting (and not allowable) value of two, for which the total mass diverges.

\subsection{Parity-dependent Kernels}

We now consider a variation of the constant kernel which is specific to the discrete case, namely a kernel in which the reaction rates depend on the parity of the sizes of the reactants but are otherwise constant. We shall therefore use the discrete notation throughout this subsection. This kernel is defined as follows:

$$
K(k, l)= \begin{cases}K & \text { if } k \text { and } l \text { are odd } \\ L & \text { if } k \text { and } l \text { are even } \\ M & \text { otherwise }\end{cases}
$$

This model was introduced in $[63,64]$. The physical justification given there was that such parity effects might arise in the diffusion-limited aggregation of alternating linear copolymers. While this seems at best questionable, there is no doubt the model provides an important testing ground for the universality assumption implicit in the use of the scaling approach. Indeed, such an alternation, within constant bounds, of reactivity according to parity is a perfect example of the sort of detail that ought to be irrelevant if we want to be able to apply scaling theory in concrete situations. It does not matter that this particular instance of deviation from constancy may not appear reasonable physically: there is every reason to believe that similar deviations may produce at least similar effects, so that the exact solutions we can find in this case are important because they teach us, to what extent the scaling theory may be expected to hold, and in what aspects it is likely to fail, once we leave the familiar ground of exactly solved systems. The discussion of the solution falls therefore squarely within the principal purpose of this review. 
It turns out to be impossible to give a full and explicit solution for the kernel (4.41). What can be done, however, is the following: on the one hand, one can prove in full generality that the kernel (4.41) satisfies scaling as defined in (3.12), with a scaling function that is, however, different from the one given in (4.11) for the constant kernel. It is also possible to give some of the non-scaling asymptotics exactly for the general kernel (4.41). On the other hand, it is also possible fully to solve some particular instances. I shall restrict myself to the case in which $L=4 M$, for which simple and completely explicit formulae are obtainable, as shown in [14]. For these, then, everything is known and can be discussed in full detail.

Let us first consider the scaling limit as defined in (3.12). As stated above, the scaling limit differs from the one obtained for the constant kernel. This invalidates to some extent the usual claims of universality of the scaling theory. However, it does not contradict what was stated in Appendix A: indeed, there, we had shown that if a kernel behaves asymptotically as a homogeneous function, then the scaling function should be given by a solution of equation (3.29). But the kernel (4.41), which oscillates for ever around the constant kernel, does not satisfy this hypothesis. Still, this change in the scaling function is quite unexpected. The scaling function $\Phi(x)$ for the kernel (4.41) is evaluated in detail in Appendix H. As we shall see, however, the set of odd and even concentrations obey completely different asymptotics. How, then, can we have scaling for the full distribution, without having to divide it into odd and even clusters? The answer lies, again, in the very particular nature of the way in which the scaling limit is defined, see (3.12). Indeed, what is considered there is the average of a function which varies on a macroscopic scale, namely the typical size $s(t)$. Such an average is clearly unaffected by any even-odd oscillations, since they are effectively averaged over. One might at first then argue that this is an artefact of an inappropriate definition. However, if one thinks about it from the purely physical point of view, the very reverse is the case: any reasonable physical measurement, say light scattering, will measure such an average and will be quite insensitive to the oscillations. In fact, the only measurement likely to feel such effects is a complete measurement of the cluster size distribution function at the level of accuracy of the monomer size unit. This is only very rarely practicable ${ }^{18}$, so that one usually has to be satisfied with the coarser measures which do not perceive the oscillations. However, the mathematical solution certainly has these oscillations, so that, as we shall see, no statement can be made stating that the individual concentrations are well approximated by the scaling form.

In Appendix $\mathrm{H}$ it is shown that both the odd and even cluster-size distributions approach scaling in the sense of (3.12) separately. This is given precise meaning in (H.11). The scaling functions $\Phi_{d}(x)$ and $\Phi_{p}(x)$ for the odd and even

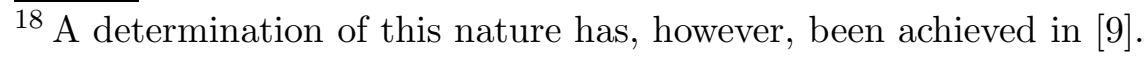


distributions respectively cannot be evaluated analytically, but the $\tau$ and $\vartheta$ exponents can. Here, as throughout in this subsection, the letter $d$ refers to the odd (dispari) clusters, whereas the letter $p$ refers to the even (pari) clusters $^{19}$. One first defines

$$
\tilde{p}_{\infty}=\frac{K-M}{L}\left[1+\sqrt{1+\frac{K L}{(K-M)^{2}}}\right] .
$$

The expressions for $\tau_{d, p}$ and $\vartheta_{p}$ are then found to be:

$$
\begin{aligned}
\tau_{d} & =1-\frac{M}{K} \tilde{p}_{\infty} \\
\tau_{p} & =\min \left[2-\frac{M}{K}+\frac{L}{K} \tilde{p}_{\infty}, 2 \tau_{d}-1\right] \\
\vartheta_{p} & =0
\end{aligned}
$$

whereas for $\vartheta_{d}$ we only know that it is positive. Note finally that from the definition of scaling given in (H.7) immediately follows that the full cluster size distribution tends to a scaling limit with the scaling function $\Phi(x)$ given by

$$
\Phi(x)=\frac{1}{2}\left[\Phi_{d}(x)+\Phi_{p}(x)\right]
$$

which is quite an extreme illustration of the fact that the scaling function need not yield a good approximation to any of the concentrations $c_{j}(t)$ at all.

Let us now turn to the non-scaling asymptotics: as usual, define the exponents

$$
\begin{aligned}
c_{2 j+1}(t) & =\text { const. } \cdot t^{-w_{d}^{\prime}} \\
c_{2 j}(t) & =\text { const. } \cdot t^{-w_{p}^{\prime}}
\end{aligned}
$$

It is then straightforward to check, as is also shortly discussed in Appendix H, that

$$
\begin{aligned}
& w_{d}^{\prime}=1+\frac{M}{K}\left(1+\tilde{p}_{\infty}\right) \\
& w_{p}^{\prime}=\min \left(1+\frac{2 M}{K} \tilde{p}_{\infty}, 2 w_{d}-1\right)
\end{aligned}
$$

$\overline{19}$ This somewhat singular notation arose first in [14]. It is unambiguous and easy to remember after some time, so I decided to stick with it here. 
Since these coincide with the values of $w_{d}$ and $w_{p}$ that are obtained from the values of (4.43) and (4.44) for $\tau_{d}$ and $\tau_{p}$ via the scaling relation (3.25), we may state that strong scaling holds.

The unexpected feature in this case is rather the non-universal nature of the result. That is, in general, one does not expect a simple odd-even oscillation in the reaction rates to produce such massive changes in the scaling function. However, one must remember that the constant kernel is in case II, which is quite non-universal in itself. In fact, as is shortly pointed out in Appendix $\mathrm{H}$, these peculiarities may be typical of case II.

\subsubsection{Two Fully Solvable Subcases}

We may cite two cases that have been solved more or less fully, which therefore allow for a test of the above computations. The first is given by

$$
L=4 M
$$

In this case a completely explicit solution has been found in [14] for the initial condition

$$
c_{j}(0)=\alpha \delta_{j, 1}+\frac{1}{2}(1-\alpha) \delta_{j, 2} .
$$

It is then given explicitly by the formulae

$$
\begin{aligned}
c_{2 j+1}(t) & =2^{-2 j}\left(\begin{array}{c}
2 j \\
j
\end{array}\right) \frac{\alpha[s(t)]^{-1 / 2}}{1+\alpha K t}\left\{1-[s(t)]^{-1}\right\}^{j} \\
c_{2 j}(t) & =\frac{2 \dot{s}(t)}{L s(t) d(t)}\left\{1-[s(t)]^{-1}\right\}^{j} \\
s(t) & =\frac{L}{\alpha(L+4 K)}(1+\alpha K t)+\frac{(\alpha-1) L+\alpha}{\alpha(L+4 K)}(1+\alpha K t)^{-L /(4 K)} .
\end{aligned}
$$

The quantity $s(t)$ defined in (4.54) is therefore the most natural definition of typical size in this system, since the forms which define $c_{2 j+1}(t)$ and $c_{2 j}(t)$ are already very close to scaling form in their exact expression. One immediately verifies from $(4.52,4.53)$ that $\tau_{d}=1 / 2$ and $\tau_{p}=0$, all of which indeed confirms exactly the results obtained in the previous subsection. Similarly, one verifies the value of zero for $\vartheta_{p}$. The exponent $\vartheta_{d}$ on the other hand, which could not be evaluated in general, has in this case the value $1 / 2$. It therefore coincides with $\tau_{d}$ in this particularly simple case, once again satisfying the Dominant Singularity Hypothesis. It is not clear in general whether this will always be the case for these kernels, however. 
Another case which can to some extent be solved exactly is the one in which

$$
M=\frac{1}{2}(K+L)
$$

In this case the kernel is of the form of a sum kernel and can be solved using the techniques mentioned in subsection 4.2. The solution obtained in [64] is extremely opaque, however, and does not yield such explicit formulae for the concentrations as are given for the case $L=4 M$. However, after some complicated calculations one obtains values of $\tau_{d}$ and $\tau_{p}$ again in full agreement with those found in the previous subsection.

\subsubsection{The addition-aggregation kernel}

In a recent paper [80] a minimal model showing anomalies very similar to those found in the parity-dependent kernel was studied. One defines constant rates, with the only additional feature that monomers are singled out and react differently from other aggregates. more precisely

$$
\begin{aligned}
& K(1,1)=\epsilon \\
& K(1, k)=K(k, 1)=1 \\
& K(k, l)=\gamma,
\end{aligned}
$$

where $k, l \geq 2$. For this model one obtains a fairly bewildering array of large time behaviours for monomers of fixed size, yet scaling in the sense in which we have defined it continues to hold, as the authors show in an Appendix. Furthermore, the scaling function is the same as for the constant kernel, thereby vindicating the usual claims of universality.

\subsection{The q-sum kernel}

In a recent paper [13], a model quite different from the classical ones was solved, namely

$$
K(k, l)=2-q^{k}-q^{l}=2-e^{-b k}-e^{-b l} .
$$

where $q=e^{-b}$ is an arbitrary number between zero and one. We shall use $q$ and $b$ interchangeably in the following. Again the model is restricted to discrete values of the masses only. This model is interesting for at least two different reasons:

(1) It is a non-trivial variant on the constant kernel, in which the reactivity of the small aggregates is diminished with respect to that of larger ones. 
The difference is only significant for rather small aggregates, at least in the general case in which $q$ is fixed and not too near to one, so that one expects no major deviations from the ordinary scaling picture. As we shall see, this is indeed the case if we interpret scaling in the sense of this paper, that is, as the convergence in first moment as defined in (3.29). On the other hand, for non-scaling asymptotics, quite unexpected results are encountered.

(2) On the other hand, it is also possible to consider a simultaneous asymptotic behaviour in which $q \rightarrow 1$ at the same time as $t \rightarrow \infty$. In this case we obtain a highly peculiar crossover situation in which the sum kernel drives the aggregation of small clusters, whereas the large clusters obey a constant kernel kinetics. We shall not have much to say about it, as the evaluation of the crossover function is a daunting task which is left to the inspired reader. It can be shown, however, that the formalism developed in 3.6 indeed yields the correct scaling variables.

In order to obtain the solution, we note that the kernel (4.57) is of the form $f(k)+f(l)$, with $f(k)$ given by $1-q^{k}$. One therefore proceeds along the same lines as described in subection 4.2 for the sum kernel, namely we use the transformation given by (4.20). This leads to the equations

$$
\frac{d \phi_{j}}{d \theta}=\sum_{j=1}^{j-1}\left(1-q^{k}\right) \phi_{k} \phi_{j-k}-\left(1-q^{j}\right) \phi_{j}
$$

These equations can in principle be solved recursively. The solution becomes very soon quite intricate, however, and it is not possible to obtain much information in this way.

The solution makes heavy use of the $q$ factorial and $q$ exponential, defined as follows

$$
\begin{aligned}
(a ; q)_{n} & =\prod_{l=0}^{n-1}\left(1-a q^{l}\right) \\
e_{q}(x) & =\prod_{l=0}^{\infty}\left(1-x q^{l}\right)^{-1} \\
& =\sum_{k=0}^{\infty} \frac{x^{r}}{(q ; q)_{r}},
\end{aligned}
$$

where the last identity is familiar from the theory of the $q$ exponential [33].

The solution, which is derived in detail in [13] is given as follows: one defines

$$
H(\zeta, \theta)=\sum_{j=1}^{\infty} \phi_{j}(\theta)\left(e^{b j \zeta}-1\right)
$$


This function satisfies a non-linear differential-difference equation, the solution of which is shortly sketched in Appendix I. The final result is given by

$$
\begin{aligned}
& H(\zeta, \theta)=-\frac{\partial}{\partial \theta} \ln [1+S(\zeta, \theta)] \\
& S(\zeta, \theta)=\frac{1}{e_{q}\left(q^{-\zeta}\right)} \sum_{r=0}^{\infty} \frac{q^{-r \zeta}}{(q ; q)_{r}}\left[\exp \left(\theta q^{r}\right)-1\right]
\end{aligned}
$$

From these results and the definition of $\theta$, see (4.20) one readily obtains

$$
t=\frac{1}{e_{q}(q)} \sum_{r=0}^{\infty} \frac{\exp \left(\theta q^{r}\right)-1}{(q ; q)_{r}}
$$

From these results essentially everything can be computed, though this can sometimes be difficult.

Let us first concentrate on the case in which $q$ is fixed. In this case, the only limits of interest are the large-time limit at fixed $j$ and the scaling limit. It is quite straightforward to obtain from (4.58) the asymptotic order of magnitude of the $c_{j}(t)$. With some additional work one obtains the more precise result

$$
c_{j}(t)=\frac{\left.t^{-\left(2-q^{j}\right.}\right)}{e_{q}(q)^{1-q^{j}}(q ; q)_{j-1}}[1+o(1)] \quad(t \rightarrow \infty)
$$

It is therefore clear that the large time asymptotics is markedly different from that of the constant kernel. In particular, the exponent $w^{\prime}$ is not even defined, since the large-time decay law depends on the size $j$ of the cluster. The only feature that remains is the fact that the exponent tends to the value 2 for large values of $j$. On the other hand, an elementary analysis shows that this behaviour is only attained when

$$
t \gg \exp \left(q^{-j}\right)
$$

which increases extremely fast with $j$. We have then obviously no contradiction with the scaling statement, which asserts that the decay goes as $t^{-2}$ as long as $j$ is of order $t$. Along similar lines, we see that the moments $M_{p}(t)$ for $p<q-1$ are dominated by $c_{1}(t)$ and hence decay as $t^{-2+q}$ instead of going as $t^{p-1}$ as predicted by scaling theory. This is the case even though, as long as $p>-1$, the scaling expression for the moments converges at the origin.

The scaling limit can also be shown to exist. This is proved by showing that the function $H(\zeta, \theta)$ has only one singularity which approaches zero as $t \rightarrow \infty$, which is a simple pole. Due to this the scaling characteristics of the constant 
kernel are obtained. The detailed proof of this fact is quite technical and is given in [65].

Let us now turn to the issues involved in the crossover regime, when $b \rightarrow 0$. This has been treated fairly extensively in [65], to which the interested reader is referred for more details. As things stand, this appears to be an instance in which the formalism developed in subsection 3.6 could be applied if we substitute $K(k, l)$ by $K(k, l) / b$, so as to satisfy the requirement that $K(1,1)$ be always of order one: indeed one has $\lambda=1$ with $b$ playing the role of the small parameter $\epsilon$ in (3.89). One therefore expects a typical size of order $e^{t}$ and scaling parameters $j e^{-t}$ and $b j$. I have not been able to carry out this evaluation successfully, however.

The results which have been obtained are the following:

(1) When bt is of order one, the sum kernel is a good approximation both for clusters of fixed size $j$ and for clusters in the scaling region. On the other hand, considerable discrepancies always exist in the large-size limit, no matter how short times are. This is related to the fact that the nearest singularity of the $q$-sum kernel is a pole, so that the $\vartheta$ exponent is zero. Similarly, we may say that moments of fixed order $p$ are, in this range, well approximated by those of the sum kernel. However, large order moments will show deviations earlier than low order moments.

(2) For sufficiently large times, all features of the constant kernel are recovered. This is an immediate consequence of the statements made about the existence of the scaling limit. However, the time needed to reach in all respects this scaling limit is surprisingly large: the necessary condition is

$$
t \gg \exp (1 / b)
$$

(3) A more accurate description of the regime lying between these two extremes would clearly be highly desirable, but I am not aware of a way to obtain such results. It can be shown that the crossover limit in the sense of subsection 3.6 exists with the scaling variables $j e^{-2 t}$ and $b j$. This is shown in Appendix J.

\subsection{The Bilinear Kernel}

The most general kernel which has been solved in classical works on the subject is the so-called bilinear kernel:

$$
K\left(m, m^{\prime}\right)=A+B\left(m+m^{\prime}\right)+C m m^{\prime} .
$$


The history of the various solutions to this kernel is too long to be told in detail. The crucial issue concerns the case in which $C>0$. As we have already stated in subsection 3.5.4, such a case, involving as it does a homogeneity degree $\lambda>1$, necessarily leads to violation of mass conservation in finite time. While it was originally believed that no solutions could be found beyond that time (see for example [76,77]), it was eventually realized $[115,58]$ that such solutions existed and were physically meaningful. The decrease in total mass was interpreted as the flow of mass to an infinite cluster, in a way quite similar to that which is observed in systems such as percolation: as the size of a finite system is taken to infinity, the total mass comprised in those clusters which are of a size that diverges as the system size may or may not be zero. In the latter case, this mass does not appear in the final bookkeeping once the system has been taken as infinite. There is hence no contradiction involved. Furthermore, from the mathematical point of view, it can in fact be shown that if the second moment of the cluster size distribution diverges, mass conservation can be violated. We shall further show explicitly how this happens in the bilinear model (4.68).

Let us first limit ourselves to the simpler model

$$
K\left(m, m^{\prime}\right)=m m^{\prime}
$$

We shall prove in Appendix M that for purposes of scaling (4.68) and (4.69) are equivalent. The possibility of solving the general kernel (4.68) exactly does, however, open new vistas concerning crossover, as we show in the next subsection.

In order to gain some insight into the behaviour of (4.69), we introduce the following generating function

$$
G(\zeta, t)=\int_{0}^{\infty} m^{\prime} c\left(m^{\prime}, t\right) e^{\zeta m^{\prime}} .
$$

This satisfies the following PDE

$$
G_{t}-\left[G-M_{1}(t)\right] G_{\zeta}=0
$$

Here we have not assumed that $M_{1}(t)$ is constant, since this hypothesis cannot be sustained over all times, though a solution to the Smoluchowski equations (2.2) with the kernel (4.69) does indeed exist for all times. Let us first consider the case in which $M_{1}(t)$ is equal to one. In this case, a straightforward application of the characteristic method yields 


$$
\begin{aligned}
G[\bar{\zeta}(t)] & =g\left(\zeta_{0}\right) \\
\bar{\zeta}(t) & =\zeta_{0}-t\left(g\left(\zeta_{0}\right)-1\right)
\end{aligned}
$$

where $g(\zeta)$ is the generating function of the initial conditions. For each value of $t$, the function $\bar{\zeta}(t)$ takes its maximum value at a value of $\zeta_{0}$ such that $g^{\prime}\left(\zeta_{0}\right)$ equal $1 / t$. Above this value $G(\zeta, t)$ is not defined. But $G(\zeta, t)$ must clearly be defined for all negative values of $\zeta$. The maximum value must therefore be at least equal to zero. But it is easy to verify, using the convexity properties of $g(\zeta)$ following from the positivity of the initial conditions, that if $t>1 / g^{\prime}(0)$, the maximum value of $\bar{\zeta}(t)$ is strictly negative, so that the function $G(\zeta, t)$ is not defined for all negative $\zeta$ for such a value of $t$. This would imply a cluster size distribution $c(m, t)$ that is exponentially increasing with $m$, an utter absurdity. For lesser values of $t$, however, everything is well-defined and the solution has all the expected properties. As an example we may cite the instance of monodisperse initial conditions, for which the exact computation is an easy excercise in contour integrals performed in Appendix K. The result is

$$
c_{j}(t)=\frac{j^{j-3}}{(j-1) !} t^{j-1} e^{-j t} .
$$

This is indeed a well-defined solution, but it is obvious that for $t>1$ the corresponding generating function stops being a solution to (4.71) with $M_{1}(t)$ equal to one, since all functions $c_{j}(t)$ decrease. In fact, these are the so-called Flory solutions of the Smoluchowski equations corresponding to (4.69). They are obtained by starting from the assumption that $M_{1}(t)$ is always equal to one, and therefore eliminating it from the Smoluchowski equations. In other words, instead of solving the original problem (2.2) for the kernel (4.69), one solves instead

$$
\dot{c}(m, t)=\frac{1}{2} \int_{0}^{\infty} m^{\prime}\left(m-m^{\prime}\right) c\left(m^{\prime}, t\right) c\left(m-m^{\prime}, t\right) d m^{\prime}-m c(m, t) .
$$

Here $M_{1}(t)$ has been set equal to one by fiat. It is then straightforward to check that (4.73) is the solution of (4.74) for all times. However, one then finds for this solution

$$
M_{1}(t)= \begin{cases}1 & (t \leq 1) \\ \exp [-\zeta(t)] & (t>1)\end{cases}
$$


where $\zeta(t)$ is given as the solution to the equation

$$
\frac{\zeta(t)}{1-e^{-\zeta(t)}}=t
$$

Clearly, (4.75) casts some doubt on the validity of (4.74) beyond $t=1$. It has, however, been defended on the following grounds: beyond the gel point, it is no longer possible to identify the finite systems and its infinite limit as we have always implicitly done so far. Rather, in the finite system, for times larger than the gelation time, we have two cluster populations: on the one hand, we have the ones that are small with respect to system size, the concentrations of which tend to a well defined limit as it is made to go to infinity. These are known as the sol particles. On the other hand, the large clusters of the finite system have sizes that go to infinity with the system size, and contain a finite portion of the mass. These so-called gel particles do interact, in the finite system, with the sol. Since, in the finite case, mass is certainly conserved, we might expect some variation of (4.74) to be derivable for the finite system. If one then goes to the limit in this fashion, the solution (4.75) is obtained for the sol for all times, and (4.76) simply tells us how the mass contained in the finite clusters decreases with time. As we shall see later, however, there exist exactly solved finite systems which tend either to the Flory solution (4.74) or to the so-called Stockmayer solution, which is the post-gel solution of (4.71). It is therefore not straightforward to decide which of the two solutions is the "correct" one. The issue is, to some extent, simplified by the fact that, in any realistic situation, the mean-field assumptions made in the derivation of the Smoluchowski equations (2.2) certainly break down near the gel point, so that the whole issue becomes irrelevant. A detailed discussion of the difference between the Flory and the Stockmayer solutions is found in [115].

Let us now derive this latter solution. The central issue is a geometric one, discussed in greater detail in Appendix $\mathrm{K}$ : it is found that the function $G(\zeta, t)$ covers the $(\zeta, t)$-plane twice and has a singularity over some curve on the $(\zeta, t)$ plane, on which the two branches of the surface join smoothly. This structure is a universal feature of all solutions of (4.71), independently of the shape of $M_{1}(t)$. However, since it is manifest that only the sheet of $G(\zeta, t)$ that connects to $\zeta=-\infty$ without crossing the singularity, has meaning as a generating function of a solution of (4.71), it is clear that we must choose $M_{1}(t)$ in such a way that the singular curve always lie above $\zeta=0$. On the other hand, after $t=1$, it follows from the previous dicussion that we cannot make $M_{1}(t)$ to remain constant. We must therefore allow a non-trivial timedependence of $M_{1}(t)$. But it is clear that such a dependence can only obtain if the second moment of the cluster size distribution diverges, that is, if the singular curve coincides with the $\zeta=0$ axis. This computation is carried out 
in detail in Appendix $\mathrm{K}$ for monodisperse initial conditions, yielding

$$
c_{j}(t)= \begin{cases}\frac{j^{j-3}}{(j-1) !} t^{j-1} e^{-j t} & (t \leq 1) \\ \frac{j^{j-3} e^{-j}}{(j-1) !} t^{-1} & (t \geq 1)\end{cases}
$$

Note that both the values and the first derivatives of the $c_{j}(t)$ are continuous, so that they really are (strong) solutions of the set of first order ordinary differential equations (2.2).

Finally let us make some remarks on the applicability of the scaling approach. As always, one must first define the sense in which we want scaling to hold. Since most of the mass is contained in clusters of size of order one, even as the gel time is approached, the usual definition of scaling given by (3.12) does not hold. As we have already stated before, the definition of second moment scaling (see (3.15) for the precise definition) is the most appropriate one. The scaling function as $t$ approaches $t_{c}$ is computed in Appendix $\mathrm{K}$, with the result

$$
\begin{aligned}
\Phi(x) & =\frac{1}{\sqrt{2 \pi \alpha}} x^{-5 / 2} e^{-x /(2 \alpha)} \\
\alpha & =\frac{g^{\prime \prime}(0)}{g^{\prime}(0)^{3}} .
\end{aligned}
$$

Two remarks are in order at this stage; First, the ordinary scaling we have just derived rests upon the finiteness of the third moment of the initial conditions. If this diverges and yet the second moment is finite, we are led to an interesting situation: the scaling behaviour is then given by different exponents, but the gel time remains finite. After this gel time, however, the asymptotic behaviour must necessarily have the exponent $-5 / 2$, since that is the only one that allows a finite rate of mass transport. In Appendix L we therefore study in detail what happens under these circumstances, as well as what happens in the case of a divergent second moment, in which case instantaneous gelation occurs.

\subsection{Crossovers in the Bilinear Kernel}

The bilinear kernel (4.68) has three variants which display crossover behaviour. Using the type of parametrization defined in (3.88), these are

$$
K\left(m, m^{\prime}\right)=1+\epsilon\left(m+m^{\prime}\right)
$$




$$
\begin{aligned}
& K\left(m, m^{\prime}\right)=1+\epsilon^{2} m m^{\prime} \\
& K\left(m, m^{\prime}\right)=m+m^{\prime}+\epsilon m m^{\prime}
\end{aligned}
$$

Let me first recall the definition stated in (3.90) of the crossover limit and the corresponding scaling function

$$
\lim _{t \rightarrow \infty} \int_{0}^{\infty} m^{\prime} c\left(m^{\prime}, y / s(t) ; t\right) f\left(\frac{m^{\prime}}{s(t)}\right) \rightarrow W \int_{0}^{\infty} x \Phi(x, y) d x
$$

Here, as in subsection 3.6, the function $c(m, \epsilon ; t)$ denotes the solution of the Smoluchowski equations (2.2) with a kernel characterized by a given value of $\epsilon$.

For the first case, the scaling function is evaluated exactly in Appendix N. It is given by the following expression

$$
\Phi(x, y)=\frac{4 y^{2}(2 x y)^{2 x y} \exp \left[-2 x y /\left(1-e^{-y}\right)\right]}{\left(1-e^{-y}\right)^{2+2 x y} \Gamma(2+2 x y)}
$$

This expression reduces to the scaling function (4.11) for the constant kernel when $y \rightarrow 0$, as of course it should. Here $s(t)$ is taken to be $t$. The limit of large $y$ is somewhat more subtle: taking the limit $x \rightarrow \infty$ and $y \rightarrow \infty$ with $x y e^{-2 y}=z$ constant, one obtains the correct limit apart from a divergent prefactor due to issues of normalization. This illustrates nicely the difficulties involved in studying the large $y$ behaviour of such functions. However, the use of (4.83) is not, of course, in the large $y$ regime, where everything is best described by the scaling function (4.39) of the sum kernel, but rather at intermediate times, for which the aggregates have already become large but not large with respect to $\epsilon^{-1}$.

On the other hand, for any finite value of $y, \Phi(x, y)$ tends to a constant as $x \rightarrow 0$, as stated in subsection 3.6. Figure 1 shows the scaling function (4.83) as a function of $x$ for various values of $y$. Note how the initial values at low $x$ are always constant, corresponding to a $\tau=0$ regime, whereas a $x^{-3 / 2}$ behaviour arises over a very extended range for large values of $y$.

It is also possible to go part of the way toward evaluating the crossover functions for one of the two other cases arising in the full bilinear kernel, namely (4.80). For the crossover from the constant to the product kernel given by (4.80), one finds the following result, the derivation of which is sketched in Appendix O: we first define

$$
q(s, y)=\frac{2}{2+s_{0}^{2}-\left(s_{0}^{2} \cos y+2 s_{0} \sin y\right)}
$$




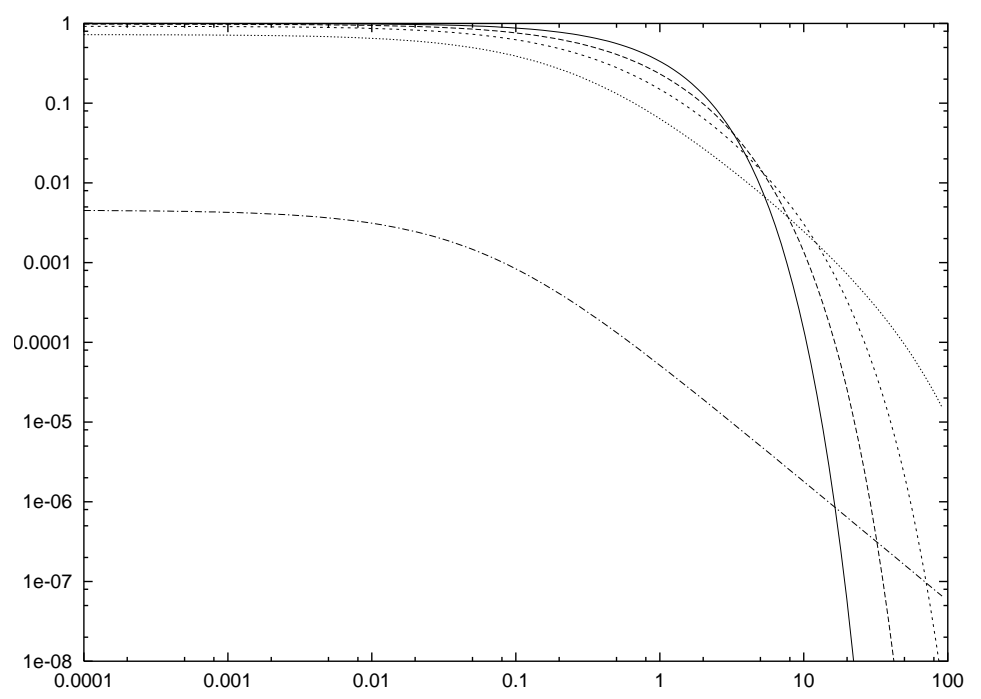

Fig. 1. The crossover scaling function is plotted as a function of $x$ for various values of $y$, namely $0.1,0.5,1,2$ and 10 . Note how a long $x^{-3 / 2}$ regime is eventually established. It requires, however, the very largest value of $y$.

$$
s=s_{0}-t+\int_{0}^{y} d y^{\prime} q\left(s, y^{\prime}\right) .
$$

Here (4.85) must first be inverted and the result substituted into (4.84) to yield the desired result. One then has for the crossover scaling function $\Phi(x, y)$

$$
q(-s, y)=\int_{0}^{\infty} x \Phi(x, y) e^{-s x} d x
$$

The analytical (or even numerical) evaluation of $\Phi(x, y)$ from these equations appears to be a non-trivial task, which is left to future work. It is easy to verify, however, that this function has a singularity at $y$ equal to $\pi / 2$, which corresponds to the gelling singularity. Indeed, as will be remembered from the definition of the crossover limit, the parameter $y$ in this case is nothing else than a rescaled time $\epsilon$. But it is easy to verify, using for example the moment equations, that the gel time for the reaction rates $(4.80)$ is of order $\pi /(2 \epsilon)$ as $\epsilon \rightarrow 0$.

On the other hand, computing the scaling function in the case (4.81) of a crossover betweeen the sum and the product kernel presents considerable difficulties. A naive approach does not appear to lead to true scaling behaviour in a straightforward way. While this is in itself a matter of some interest, it should be left as a subject for future work, as the issue is not settled. 


\subsection{Finite Systems}

It has been shown by Lushnikov and Piskunov [70] that the following finite versions of the classical reaction kernels $K_{1}, K_{2}$ and $K_{3}$ as defined in (4.1, 4.2, 4.3) can be solved exactly at least in the discrete case:

$$
K_{\alpha}^{(N)}(k, l)=\left\{\begin{array}{cc}
K_{\alpha}(k, l) & (\min (k, l) \leq N) \\
0 & (\min (k, l)>N)
\end{array}\right.
$$

These have all been solved exactly in [70]. We shall first discuss in some detail the case of $K_{1}^{(N)}(k, l)$, showing how the exact solution can be obtained and discussing its behaviour also from the point of view of the crossover theory devfeloped in the previous section. We shortly state the exact solution for $K_{2}^{(N)}(k, l)$ and go on to analyze the limiting behaviour of $K_{3}^{(N)}(k, l)$. This last is of particular interest, as it tends to the kernel $K_{3}(k, l)$ as $N \rightarrow \infty$, which displays gelation. On the other hand, it is obvious that $K_{3}^{(N)}(k, l)$ cannot display such a phenomenon. As we shall see, one can show that $K_{3}^{(N)}(k, l)$ leads to the Stockmayer kinetics as defined in subsection (4.5), that is, the exact solution to the equations defined by the kernel $K_{3}^{(N)}(k, l)$ tend to the solution of the full Smoluchowski equations, not to those for which one has performed the substitution of the first moment by the constant value of the total mass (Flory solution). On the other hand, as can also easily be shown (see [4]) the following finite version of $K_{3}(k, l)$ will lead to the Flory solution

$$
\bar{K}_{3}^{(N)}(k, l)=\left\{\begin{array}{cc}
K_{3}(k, l) & \max (k, l) \leq N \\
0 & \max (k, l)>N
\end{array}\right.
$$

We therefore have the very remarkable phenomenon, that the limiting behaviour of the finite systems depends crucially on the nature of the cutoff. The reason for this has been pointed out already by Ziff et al. [117,24], where it is shown that the Stockmayer solution corresponds to a situation in which large aggregates do not react with small ones, whereas the Flory solution correpsonds to the case in which they do. Here, by "large", I mean comparable to the size of the cutoff. In this case, it is understandable that $K_{3}^{(N)}$ should tend to the Stockmayer solution, since reactions between large particles are strongly restricted, whereas in $\bar{K}_{3}^{(N)}$ this is not the case.

At this stage it is important to clear up a possible misunderstanding: with the above kernels one aims to study the gelation transition by viewing it as the limit of a set of non-gelling kernels, which represent systems with only a finite number of different reacting species. This should be sharply contrasted 
with the regularization induced by considering only a finite number of reactive aggregates in the first place. The effects of such an approximation are much more complex, since one must then take into account the qualitatively new phenomenon of fluctuations in the aggregate numbers. This has been done by van Dongen and Ernst in [104], which presents the whole issue with great clarity. This, however, belongs to those interesting subjects, the treatment of which falls outside of this review.

\subsubsection{The Finite Constant Kernel}

We shall here treat the case of the kernel $K_{1}^{(N)}(k, l)$ as defined by $(4.87)$. The exact solution can only be found in a straightforward way for the case of monodisperse initial conditions, which is why we have limited ourselves to the discrete case. The solution in this case is given by

$$
\begin{aligned}
c_{j}(t) & =\theta^{j-1} \exp \left[-2 \sum_{k=0}^{N} \frac{\theta^{k}}{k}\right], \\
t & =2 \int_{0}^{\theta} d y \exp \left[2 \sum_{k=0}^{N} \frac{y^{k}}{k}\right] .
\end{aligned}
$$

For completeness' sake the derivation of this solution is given in Appendix P. It should, by the way, be emphasized that the solution here given is, in a sense, incomplete: Indeed, the functions $c_{j}(t)$ for $N<j \leq 2 N$ are different from zero and are not given by the expressions (4.89). These are inactive clusters formed by the aggregation of clusters of size less than $N$. Eventually, of course, all the mass will come to be concentrated in those clusters, whereas the mass contained in the active clusters goes to zero.

Qualitatively, one sees that the solution behaves as follows: as long as $\theta$ remains significantly less than one, one finds that the solution given by (4.89) is very close to the exact solution of the constant kernel given in (4.10). On the other hand, as soon as $\theta$ becomes significantly larger than one, the concentration profile becomes exponentially increasing and a large amount of the total mass finds itself in the inactive clusters. It also follows from (4.90). that the time at which this change in behaviour takes place is of the order of the cutoff $N$.

It is, of course, quite obvious that such a kernel in the limit of large $N$ offers a good example of a crossover situation, as discussed in a similar case in [111]. From the exact solution we may obtain the crossover behaviour as follows: first define a scaling variable $\xi$ given by

$$
\xi=N(1-\theta) .
$$


We now take note of the following identity valid in the scaling regime in which $\xi$ is of order one, also shown in Appendix P:

$$
\sum_{k=1}^{N} \frac{\theta^{k}}{k}= \begin{cases}-E_{1}(\xi)-\ln \xi & (\xi>0) \\ \operatorname{Ei}(|\xi|)-\ln |\xi| & (\xi<0)\end{cases}
$$

where the exponential integrals $E_{1}(x)$ and $E i(x)$ are defined as in [1]. If one now substitutes these expressions in (4.89) and (4.90) one eventually obtains

$$
\begin{aligned}
& c_{j}(t)=\left\{\begin{array}{cc}
t^{-2} \xi^{2}\left(\frac{t}{N}\right)^{2} \exp \left(-x \xi t / N+2 E_{1}(\xi)\right) & (\xi>0) \\
t^{-2} \xi^{2}\left(\frac{t}{N}\right)^{2} \exp (-x \xi t / N-2 \operatorname{Ei}(|\xi|)) & (\xi<0)
\end{array}\right. \\
& t / N= \begin{cases}2 \int_{\xi}^{\infty} \exp \left[-2 E_{1}(\eta)\right] \frac{d \eta}{\eta^{2}} & (\xi>0) \\
2 \int_{-\infty}^{|\xi|} \exp [+2 \operatorname{Ei}(\eta)] \frac{d \eta}{\eta^{2}} & (\xi<0),\end{cases}
\end{aligned}
$$

where $x$ is the scaling variable $j / t$. We see therefore that $t^{2} c_{j}(t)$ depend only on the scaling variables $x$ and $t / N$, or equivalently $\xi$. The scaling variables required are indeed those predicted by the crossover theory developed in subsection 3.6: the exponent $\lambda$ is equal to zero and $\epsilon$ is equal to $1 / N$, as is readily seen if we write $K_{1}^{(N)}$ as follows

$$
K_{1}^{(N)}(k, l)=\chi_{[0,1]}\left(\frac{k}{N}\right) \chi_{[0,1]}\left(\frac{l}{N}\right)
$$

where $\chi_{[0,1]}(x)$ is the characteristic function of the unit interval.

As to the qualitative behaviour of the crossover function described by (4.93) and (4.94), it must be admitted that is is not wholly transparent. However, it does describe the transition between an exponentially decreasing cluster size distribution and an exponentially growing one as $\xi$ changes sign, which it clearly must do, in view of (4.94): as soon as $t / N$ is larger than the (convergent) integral from $\xi$ to $\infty$ given on the right-hand side, $\xi$ must become negative, so that the concentration profile becomes exponentially increasing. Note further that the scaling picture shown here implies that for times of order $N$, that is in the scaling regime, the total mass in the active range is still of order one, and can be given in terms of the scaling variables by the following expression

$$
M_{1}(t)=\left\{\begin{array}{cc}
\exp \left[2 E_{1}(\xi)\right] & (\xi>0) \\
\exp [-2 \operatorname{Ei}(|\xi|)] & (\xi<0)
\end{array}\right.
$$




\subsubsection{The Finite Sum Kernel}

For the kernel $K_{2}^{(N)}(k, l)$, the results are entirely similar. I therefore limit myself to stating the exact solution, which is given by

$$
\begin{aligned}
& \theta=\frac{1}{\Lambda} \exp \left[-\int_{\Lambda}^{\infty} d w \frac{\sum_{k=1}^{N} a_{k} w^{-(k+1)}}{1-\sum_{k=1}^{N} a_{k} w^{-k}}\right] \\
& t=\int_{0}^{\theta} \frac{d \theta^{\prime}}{1-\sum_{k=1}^{N} a_{k} \Lambda\left(\theta^{\prime}\right)^{-k}} \\
& c_{j}(t)=\frac{a_{j}}{\theta \Lambda^{j}}\left(1-\sum_{k=1}^{N} a_{k} \Lambda(\theta)^{-k}\right) \\
& a_{k}=\frac{k^{k-2}}{(k-1) !}
\end{aligned}
$$

The derivation of this solution proceeds on exactly the same lines as that of the constant kernel and is given in Appendix Q. The detailed analysis of the crossover is left as an excercise to the enthusiastic reader. Concerning the asymptotic behaviour, however, one can make the following observations: as follows from the computations in Appendix $\mathrm{Q}$, for $N=\infty, \sum_{k=1}^{\infty} a_{k} w^{-k}$ has a singularity at $w=1$, where it has a value of one. There is hence for every finite $N$ a value $w_{c}(N)<1$ such that

$$
\sum_{k=1}^{N} a_{k} w_{c}(N)^{-k}=1
$$

which is the limiting value to which $\Lambda$ tends as $\theta \rightarrow \infty$. Since the singularity in the integral representing $\theta$ is a simple pole at $\Lambda=w_{c}(N), \theta$ diverges logarithmically with $t$, in contradistinction to the $N=\infty$ case, for which it saturates at one. As for the constant kernel, we must distinguish between times for which the simple pole at $w_{c}(N)$ dominates and earlier times., for which we are far enough from the singularity for the finite sum to be well approximated by the infinite sum.

\subsubsection{The Finite Product Kernel}

The exact solution for $K_{3}^{(N)}(k, l)$ is surprisingly simple to obtain using the same approach as in the previous two cases. The final result is given by:

$$
c_{j}(t)=\frac{a_{j} \Lambda^{j}}{t}
$$




$$
\begin{aligned}
& t=\Lambda \exp \left[\int_{0}^{\Lambda} \frac{\sum_{k=1}^{N} a_{k} z^{k-1}}{1-\sum_{k=1}^{N} a_{k} z^{k}} d z\right] \\
& a_{j}=\frac{j^{j-2} e^{-j}}{(j-1) !}
\end{aligned}
$$

Using these formulae, one sees that $\Lambda$ grows monotonically with time starting from zero. If $N$ is infinite then, upon reaching the critical value $e^{-1}, \Lambda$ will yield a finite value of the time, but will not be able to grow beyond this value, since the integrand then has an integrable singularity. This critical time is indeed the gel time and is, in this case, equal to one. Beyond this time, the above equations do not quite state unambiguously what happens, but a closer look at the derivation shows that $\Lambda$ remains at its critical value of $e^{-1}$. We have therefore rederived the Stockmayer solution in this way.

If, on the other hand, we take $N$ to be finite, the behaviour becomes qualitatively quite different: indeed, the nearest singularity of the integrand on the positive real axis is now a simple pole, since the denominator is a polynomial. Since this polynomial approximates the corresponding analytic function quite well, we see that this singularity lies beyond the value $e^{-1}$ by an amount of order $1 / N$. THerefore, as time goes on, $\Lambda$ will approach ever nearer to this pole without ever reaching it. It then follows that the finite aggregates (those having $j \ll N$ ) are given for all times very nearly by the Stockmayer solution. This therefore shows our claim, that the kernel $\left.K_{(}^{(N)} k, l\right)$ yields in the limit $N \rightarrow \infty$ the Stockmayer kinetics for the finite aggregates. Note, on the other hand, that for times larger than $t_{c}$, the aggregates of size of order $n$ will be significantly different from the value predicted by the infinite model, since $\Lambda$ is distant by a quantity of order $1 / N$ from its theoretical value, thus implying a change for those aggregates by a factor of order one.

Let us now look at the kernel $\bar{K}_{3}^{(N)}(k, l)$. Since it is bounded from above by const. $\cdot N(i+j)$ it cannot display gelation, so that, if one considers all aggregates, mass must be conserved. From this follows that the kinetic equations (2.2) for $\bar{K}_{3}^{(N)}(k, l)$ for the aggregates of size $1 \leq j \leq N$ read

$$
\dot{c}_{j}=\frac{1}{2} \sum_{k=1}^{j-1} k(j-k) c_{k} c_{j-k}-j c_{j}
$$

which are manifestly the same as the Flory equations (4.74) as pointed out by Bak and Heilman in [4]. They therefore have the same solution for the active clusters, so that the solutions of (4.100) indeed straightforwardly tend to the Flory solution.

We are hence in the highly peculiar situation that the limiting dynamics depends on the sequence by which the infinite system is approached. It is intu- 
itively clear that this can only happen in a gelling system. The issue at hand is that of the interaction between finite aggregates (having a size $j \ll N$ ) and "gel aggregates", of size of order $N$. In fact, it follows from a careful study of the global existence results proved in [58] that such a situation can never arise in the situation studied there, namely whenever the kernel is bounded from above as follows:

$$
K(k, l) \leq r_{k} r_{l} \quad\left(r_{k}=o(k)\right)
$$

and, in fact, more generally whenever $\nu<1$ with $\nu$ defined as in (3.5). The peculiar phenomena described above can therefore only arise for a kernel with $\nu=1$ and for which gelation occurs. (It has been shown by van Dongen [107] that kernels with $\nu>1$ have instantaneous gelation, and in fact their solutions may in fact perhaps not exist at all). The intuitive reason for this is as follows: when $\nu<1$, the reaction rates between a $j$-mer and an $N$-mer, with $N \gg j$ is of order $N^{\nu}$. If we now average this reaction rate over all gel aggregates and use the fact that the gel mass is finite, we see that the average reaction rate tends to zero. It is therefore essential that $\nu=1$ for these pathologies to occur. This may perhaps diminish the sense of urgency in finding a "solution" to these problems. The above results do show, however, that no resolution can be expected from the consideration of finite systems when $\nu=1$.

\subsection{Pure Three-body Reactions}

It is fairly straightforward to generalize the methods described in the preceding subsections to higher order reactions. Let us first describe what has already been done in the literature. In $[42,43]$ Jiang et al. described the behaviour of the following $n$-particle kernel:

$$
K\left(m_{1}, \ldots m_{n}\right)=\prod_{l=1}^{n}\left(A m_{l}+B\right)
$$

These show gelation with exponents $z=-2$ and $\tau=2.5$. Furthermore, the case of monodisperse initial conditions can be solved exactly in this case as well. These results are obtained using an extension of the techniques described in [58]. This agrees quite well with the overall picture given in Section 3 (see in particular subsection 3.7), that as far as scaling properties are concerned, many-body reaactions do not involve major changes from binary, apart from replacing $\lambda$ by $\lambda-(n-2)$. It can also straightforwardly be verified that the constant kernel reaches scaling from arbitrary initial conditions with $\tau=0$ and $z=1 /(n-1)$. In these examples we therefore do not see any variation from the expected picture. 
We now turn to the two other examples given in (3.134) and (3.135). These, as we stated before, show some significant peculiarities. $K_{1}$, in which the rates are given by $m_{1}+m_{2}+m_{3}$, can be straightforwardly solved by an extension of the techniques described for the sum kernel, see [50]. As usual, let us define the generating function:

$$
F(\zeta, t)=\int_{0}^{\infty} c(m, t) e^{m \zeta}
$$

one finds the following $\mathrm{PDE}$ for $F(\zeta, t)$

$$
\begin{aligned}
\partial_{t} F & =\frac{1}{2}\left[F^{2}-S(t)^{2}\right] F_{\zeta}-S(t) F \\
S(t) & =F(\zeta=0 ; t) \\
f(\zeta) & =F(\zeta ; t=0)
\end{aligned}
$$

These equations can again be solved via the characteristic function technique, as discussed in appendix $\mathrm{R}$, with the following result

$$
\begin{aligned}
F(\zeta, t) & =\frac{f\left(\zeta_{0}\right)}{1+f(0) t} \\
\zeta & =\frac{\zeta_{0}}{1+t f(0)}-\frac{1}{2} f^{\prime \prime}(0) \zeta_{0}^{2}
\end{aligned}
$$

From this, the solution for monodisperse initial conditions can be obtained explicitly and is given by

$$
\begin{aligned}
c_{2 k+1}(t) & =\frac{(2 k+1)^{k-1}}{2^{k} k !} \frac{1}{1+t}\left(\frac{t}{1+t}\right)^{k} \exp \left(-\frac{2 k+1}{2} \frac{t}{1+t}\right) \\
c_{2 k}(t) & =0 .
\end{aligned}
$$

One finds that the typical size $s(t)$ grows as $t^{2}$. This leads immediately to the following scaling function:

$$
\Phi(x)=\frac{x^{-3 / 2} e^{-x / 4}}{2 \sqrt{\pi}} .
$$

The remarkable result, however, is not in the scaling function, but rather in the rate of growth of the typical size. Ordinarily, as we have seen in many occasions, this quantity is not difficult to find out and its value rests on very simple-minded, and hence very robust, scaling arguments. Here, however, this is not the case: a perfectly straightforward scaling theory predicts that $z=1$ for this kernel, but one finds through an exact solution that $z=2$. It is 
also easy to see, using the same kind of techniques that were developed for other systems, that this scaling function is in fact valid for arbitrary initial conditions. We see, therefore, that this is an example of the phenomenon discussed earlier, namely in subsection 3.7: one indeed readily verifies that the condition $\tau-\mu<1$, which is necessary for the validity of the scaling equation (3.129) for three-body reactions is here violated. Since the derivation of (3.129) fails because of a divergence of the integrals involved, we see that the expression for the typical size will also be given incorrectly: in fact, the real typical size is larger than the one predicted by (3.125). The following rough argument may help to understand what is happening: if we cut the integrals appearing in (3.129) at a lower limit of $1 / s(t)$, these become time dependent quantities, diverging as $s(t)^{1 / 2}$, thus leading to a modified version of the equation (3.125) for $s(t)$, namely

$$
\dot{s}(t) \propto s(t)^{\lambda-1} s(t)^{1 / 2} \propto s(t)^{1 / 2},
$$

which yields the correct large-time behaviour for $s(t)$.

It is also straightforward to verify that, for strongly polydisperse initial conditions, that is, if $c(m, 0)$ goes as $m^{-\alpha}$ with $2<\alpha<3$, then both $z$ and $\tau$ vary continuously with $\alpha$ (see Appendix R). This is reminiscent of similar properties for the (two-body) sum kernel. Nevertheless, the peculiar behaviour of $z$ is much more striking in this three-body case, since we are dealing with continuously variable exponents, not a continuously variable exponential growth rate. Of course, since the various exponents are undetermined by our general considerations, there is no a priori reason why they should not vary continuously with the nature of the initial condition.

Let us now consider instead the kernel $K_{2}$ given by

$$
K_{2}(k, l, m)=k l+k m+l m .
$$

In order to avoid tedious repetitions, let us use a simpler though less complete method of solution: we write down the equations for the various moments $M_{p}$ as follows

$$
\dot{M}_{p}=\frac{1}{2} \sum_{\substack{p_{1}, p_{2}, p_{3} \\ p_{1}+p_{2}+p_{3}=p}} \frac{p !}{p_{1} ! p_{2} ! p_{3} !} M_{p_{1}+1} M_{p_{2}+1} M_{p_{3}}
$$

where the primes indicates that the sum runs only over those indices for which no two of the $p_{i}$ simultaneously vanish. From this follows in particular

$$
\dot{M}_{0}=-\frac{1}{2} M_{0}
$$




$$
\dot{M}_{2}=M_{2}^{2} M_{0}+2 M_{2} .
$$

These equations can be solved exactly. However, it is almost immediately apparent, without any further work, that $M_{2}$ diverges at finite time. This is confirmed by the exact solution, which reads, for the case of monodisperse initial conditions:

$$
M_{2}(t)=\frac{e^{2 t}}{2-e^{t}} .
$$

We therefore find that this kernel gels, although the straightforward formula for $z$ leads us to expect an exponential growth of the typical size. From this follows, incidentally, that a theorem such as that proved in [112] cannot be generalized in the expected way to the pure three-body case.

Summarizing, we have given two examples for which the naive scaling arguments give a value for the growth exponent $z$ different from $1 /\left(2-\lambda_{3}\right)$, which is the result of the straightforward scaling approach. We may now ask: What happens in the more realistic case, in which the three-body term perturbs a given two-body aggregation process. In the next subsection, we shall consider the cases in which the kernel $K_{1}$ perturbs the constant kernel, and the kernel $K_{2}$ perturbs the sum kernel. Thus in both cases we consider the case in which the two-body reaction rate have the same growth exponent $z$ as $1 /\left(2-\lambda_{3}\right)$ for the three-body kernel. In other words, we ask whether the naive value for the growth exponent may not play some role when the three-body dynamics is taken together with a corresponding two-body aggregation. As we shall see, the answer is (in part) affirmative.

\subsection{Three-body Reactions Perturbing Two-body Aggregation}

Let us now consider the case in which both two and three-body reactions occur. We therefore consider the system of equations

$$
\begin{aligned}
\dot{c}(m, t)= & \frac{1}{2} \int_{0}^{\infty} d m_{1} d m_{2} K^{(2)}\left(m_{1}, m_{2}\right) c\left(m_{1}, t\right) c\left(m_{2}, t\right) \times \\
& {\left[\delta\left(m_{1}+m_{2}-m\right)-\delta\left(m_{1}-m\right)-\delta\left(m_{2}-m\right)\right]+} \\
& \left.+\frac{\alpha}{6} \int_{0}^{\infty} d m_{1} d m_{2} d m_{3} K^{(3)}\left(m_{1}, m_{2}, m_{3}\right) c\left(m_{1}, t\right) c\left(m_{2}, t\right)\right) c\left(m_{3}, t\right) \times \\
& {\left[\delta\left(m_{1}+m_{2}+m_{3}-m\right)-\delta\left(m_{1}-m\right)-\delta\left(m_{2}-m\right)-\delta\left(m_{3}-m\right)\right] }
\end{aligned}
$$


Here we shall discuss the two following cases

$$
\begin{aligned}
K^{(2)}\left(m_{1}, m_{2}\right) & =1 & K^{(3)}\left(m_{1}, m_{2}, m_{3}\right) & =m_{1}+m_{2}+m_{3} \\
K^{(2)}\left(m_{1}, m_{2}\right) & =m_{1}+m_{2} & K^{(3)}\left(m_{1}, m_{2}, m_{3}\right) & =m_{1} m_{2}+m_{1} m_{2}+m_{2} m_{3}
\end{aligned}
$$

In Appendix S we show in some detail using the method of characteristics that the first case does indeed satisfy scaling with the following exponents

$$
z= \begin{cases}1 & \left(\alpha<\frac{1}{2}\right) \\ \frac{4 \alpha}{1+2 \alpha} & \left(\alpha \geq \frac{1}{2}\right)\end{cases}
$$

This can also be seen more easily using the method of moments. Indeed, one finds

$$
\begin{aligned}
& \dot{M}_{0}=-\left(\frac{1}{2}+\alpha\right) M_{0}^{2} \\
& \dot{M}_{2}=1+\alpha+2 \alpha M_{0} M_{2} .
\end{aligned}
$$

From this the values of $z$ stated in (4.118) follow straightforwardly if we assume scaling instead of proving it. This shows therefore that when the sum kernel perturbs a two-body kernel, it behaves very much as if it had the value of $z$ predicted by a naive scaling theory: that is, it does not affect the growth exponent of a two-body kernel which itself has $z$ equal to one unless the strength of the perturbation reaches a certain threshold value. Above this threshold value, on the other hand, it does affect the growth exponent, but only to the extent of making it continuously variable, rather than imposing its own value. This last only happens in the limit in which the three-body perturbation becomes infinitely strong. Yet another peculiar feature of this model is the following: it is an instance in which adding to a given aggregation mechanism, namely the three-body kernel, a very strong second mechanism, namely the constant kernel, actually leads to a slowing down of the first mechanism. This should be compared with a conjecture framed in [60] in which it was hypothesized that if

$$
K_{1}\left(m, m^{\prime}\right)>K_{2}\left(m, m^{\prime}\right)
$$

for all $m, m^{\prime}$, then the typical size of the system corresponding to $K_{1}$ would grow faster than the one corresponding to $K_{2}$. Counterexamples were given in [12], but they involved channeling particles towards non-reactive states. The above example, although not strictly to the point since it involves ternary reactions, certainly shows the need for great care in making such claims. 
From the results of Appendix $\mathrm{S}$ we also obtain the scaling function $\Phi(x)$, albeit in a very implicit form. However, this allows to estimate its small $x$ behaviour and hence the exponent $\tau$. One obtains the result

$$
\tau= \begin{cases}1 & (\alpha<1 / 2) \\ \frac{3}{2} & (\alpha>1 / 2)\end{cases}
$$

Note that from this and the scaling relation (3.25) follows that, for $\alpha<1 / 2, w$ is equal to one. However, as is easy to see, for monomers (and more generally speaking, whenever $j$ is held fixed and $t$ goes to infinity) the decay exponent $w^{\prime}$ is given by

$$
w^{\prime}=\frac{2+2 \alpha}{1+2 \alpha} \text {. }
$$

We therefore see that in this model strong scaling is violated. It is arguable whether this is a more "natural" model than the $q$ model as an example of this phenomenon. In the $q$ model, we require a non-homogeneous kernel, whereas here we have a homogeneous two-body kernel perturbed by a three body kernel of a degree of homogeneity which, according to a naive scaling argument, should yield the same growth rate. The type of violation involved is also, in a sense, less drastic, since the $w^{\prime}$ exponent is at least defined independently of $j$.

On the other hand, writing down the moment equations for the sum kernel perturbed by the three-body sum-of-products kernels yields a gelling system: this is easily seen by examining the equations for $M_{2}(t)$ and $M_{0}(t)$, which only involve one another and can be solved elementarily. Again, it is easy to verify that the solution for the second moment diverges no matter how small $\alpha$ is. We see that the behaviour is sometimes the one that would have been expected on naive grounds, but that one cannot count on it.

\subsection{Constant Kernel with Production Term}

The constant kernel with a time-independent production term can also be solved exactly. The results are quite instructive, so we present them here in some detail. The equations are

$$
\begin{aligned}
\partial_{t} c(m, t)= & \frac{1}{2} \int_{0}^{\infty} c\left(m_{1}\right) c\left(m_{2}\right)\left[\delta\left(m-m_{1}-m_{2}\right)-\right. \\
& \left.\delta\left(m-m_{1}\right)-\delta\left(m-m_{2}\right)\right] d m_{1} d m_{2}+P \delta\left(m-m_{0}\right) .
\end{aligned}
$$


For simplicity, we assume that no aggregates are present at time zero. From this follows that only aggregates with mass an integer multiple of $m_{0}$ are produced, which allows to use the notation for discrete initial conditions.

Under these circumstances a straightforward application of the generating function technique yields the following solution: define

$$
G(\zeta, t)=\sum_{k=1}^{\infty} c_{k}(t)\left(e^{k \zeta}-1\right)
$$

One then obtains after a straightforward calculation

$$
G=-\sqrt{2 P\left(1-e^{\zeta}\right)} \tanh \left(\frac{1}{2} t \sqrt{2 P\left(1-e^{\zeta}\right)}\right)
$$

Note how this function is meromorphic with a countable set of simple poles on the positive real axis which crowd toward the origin as $t \rightarrow \infty$. Since all singularities are simple poles, one finds that $\vartheta=0$, whereas, as we have seen before, $\tau=3 / 2$, thereby violating the Dominant Singularity Hypothesis.

We now use the identity

$$
\tanh x=x \sum_{l=-\infty}^{\infty} \frac{1}{x^{2}+(2 l+1)^{2} \pi^{2} / 4}
$$

in order to find the solution

$$
c_{k}(t)=\frac{\pi^{2}}{P t^{3}} \sum_{l=-\infty}^{\infty}(2 l+1)^{2}\left[1+\frac{(2 l+1)^{2} \pi^{2}}{2 P t^{2}}\right]^{-(k+1)},
$$

which yields the various results stated in the previous section in a rather straightforward way: indeed, if we let $t \rightarrow \infty$ at fixed values of $k$, one obtains a constant value, since (4.127) is then a Riemann sum which can be replaced by an integral, namely

$$
\lim _{t \rightarrow \infty} c_{k}(t)=\frac{\sqrt{2 P}}{\pi} \int_{-\infty}^{\infty} \frac{x^{2}}{\left(1+x^{2}\right)^{k+1}} d x=\sqrt{\frac{2 P}{\pi}} \frac{\Gamma\left(k-\frac{1}{2}\right)}{\Gamma(k+1)}
$$

This confirms the general results that such models tend to a stationary value, as well as the specific value for the limiting concentrations. Indeed these are well-known to be given by the second expression in (4.128).

However, from (4.127) one also finds the general scaling form for large sizes and large times. This goes beyond the well-known results for the stationary 
distribution, giving a fairly complete overview of the dynamical behaviour. The result is readily obtained by noting that, when $k$ is of the order of $t^{2}$, only the first few terms of the infinite sum in (4.127) contribute, and that these can be approximated accordingly. However, a simpler approach is the following: remembering the expression (3.14) for the scaling function $\Phi(x)$, we obtain the following expression for the Laplace transform of $\Phi(x)$ :

$$
\int_{0}^{\infty} d x \Phi(x)\left(e^{-\rho x}-1\right)=-\sqrt{2 s} \tanh \sqrt{\frac{s}{2}}
$$

from which it is straightforward to obtain the scaling function.

\subsection{The constant kernel with a localized source term}

Let us now consider the case in which one has the constant kernel with a localized source term and a diffusion term, that is

$$
\begin{aligned}
\partial_{t} c(m, \vec{r} ; t)= & \frac{1}{2} \int_{0}^{\infty} K\left(m_{1}, m_{2}\right) c\left(m_{1}\right) c\left(m_{2}\right)\left[\delta\left(m-m_{1}-m_{2}\right)-\right. \\
- & \left.\delta\left(m-m_{1}\right)-\delta\left(m-m_{2}\right)\right] d m_{1} d m_{2}+ \\
& +P \delta\left(m-m_{0}\right) \delta(\vec{r})+D \Delta c .
\end{aligned}
$$

We essentially follow the treatment given in [17]. In this case, it is an obvious observation that the moments obey a closed set of equations, namely

$$
\dot{M}_{p}(\vec{r}, t)=\frac{1}{2} \sum_{q=1}^{p-1}\left(\begin{array}{l}
p \\
q
\end{array}\right) M_{q} M_{p-q}+P m_{0}^{p} \delta(\vec{r})+D \Delta M_{p} .
$$

These are diffusion equations with a recursively given inhomogeneity, so they

can clearly be solved iteratively. Again, however, the explicit expressions rapidly become so unwieldy that they do not yield any understanding of the system. It is nevertheless possible to gain some insight by looking for scaling solutions of (4.131) as follows: define

$$
M_{p}(t)=t^{\alpha_{p}} \Psi_{p}\left(\frac{|\vec{r}|}{\sqrt{t}}\right) .
$$

Matching powers on both sides of (4.131) and noting that

$$
\int M_{1}(\vec{r}, t) d \vec{r}=P m_{0} t
$$


which sets the value of $\alpha_{1}$ to $1-d / 2$, one finally obtains

$$
\alpha_{p}=(2-d / 2) p-1
$$

The fact that the $\alpha_{p}$ increase as $p$ is crucial for the approximations to be consistent: indeed, the production term in (4.131) contributes to the total mass $M_{1}(\vec{r}, t)$, and is neglected for higher values of $p$. This is only valid for $d \leq 4$, so that the upper crittical dimension of this system is 4 . Indeed, for $d>4$, it can be verified by a similar power counting argument that the aggregation terms can be discarded and the whole problem reduces to diffusion with a constant source. On the other hand, as we shall see in Section 5, for dimensions one and two the hypotheses of mean-field theory are bound to fail at some stage. This does not mean that the theory developed here is never applicable: if the reaction probability upon contact is very small, there will be a large intermediate regime for which the theory just developed will apply. However, eventually, it will fail, and the issues discussed in Section 5 become of central importance.

A considerable simplification occurs in (4.130) if the time-dependence of the 1.h.s. is neglected or, in other words, if we consider the stationary approximation. This is in fact what was done in [17]: it is shown there that the $c(m, r)$ have a scaling form defined as follows:

$$
c(m, r)=m^{-\tau} \Phi\left(m / r^{\tilde{z}}\right)
$$

where the exponents $\tau$ and $\tilde{z}$ are given by

$$
\begin{aligned}
& \tilde{z}=4-d \\
& \tau=\frac{d-6}{d-4}
\end{aligned}
$$

The reader is referred to the original paper for further details. In particular, an exact solution can be obtained for the one-dimensional mean-field case, as well as for the diffusion-limited case, for which techniques analogous to those to be discussed in the next section are necessary.

\section{Beyond Mean-field}

As has been stated many times, the mean-field equations (2.2) are only a valid description if no spatial correlations between the aggregates arise. The reason for this requirement is clear enough. Implicitly, we have always had the following picture in mind: aggregates, in order to grow, must first be 
brought into contact by some transport mechanism, and then react. Both the efficiency of the former and that of the latter may depend in an arbitrary manner on the masses $m$ and $m^{\prime}$ of the two reacting aggregates. The reaction rate matrix $K\left(m, m^{\prime}\right)$ is therefore a product of a transport-related collision crosssection $k\left(m, m^{\prime}\right)$ and a reaction efficiency upon contact given by some matrix $\sigma\left(m, m^{\prime}\right)$. In this picture, however, we take it for granted that those pairs of aggregates which the transport mechanism is likely to bring together represent an unbiased sample of all aggregates, in other words, that no correlations between aggregates exist on the scale determining their transport.

This is, as we shall see in the following, a non-trivial assumption. More exactly, we shall see that in the majority of physically relevant cases, it is likely that this hypothesis cannot be sustained for large times. On the other hand, in very many systems, there is a large regime of intermediate times for which the mean-field theory described in the first part of this paper is valid. This is due to the fact that, for many systems of practical relevance, the limiting factor in the reaction is not the transport mechanism itself, but rather the efficiency of reaction upon contact. In that case, it is straightforward to see that, over the times necessary to produce one single aggregation event, the transport mechanism will have randomized the environment of the reacting pair so efficiently that the mean-field assumption is unproblematic. If, on the other hand, the transport mechanism is the rate-limiting factor, then matters are much more difficult: as we shall see, the transport and the reaction mechanisms may then collaborate to create correlations between particles, leading to a situation in which mean-field theory fails. In the following, we shall therefore not be concerned with solutions of (2.2) any more. Rather, we shall consider specific models for irreversible aggregation, in which a given transport mechanism is specified, as well as a reaction mechanism. We then proceed to analyze the cluster size ditributions generated by these models, comparing them with possible solutions of (2.2) and using the scaling techniques developed in the rest of this paper to characterize the behaviour of these distributions.

\subsection{Diffusion-limited Cluster-Cluster Aggregation}

The simplest model for irreversible aggregation, inspired from the physics of colloids, for which the transport mechanism is Brownian diffusion, is known as the Particle Coalescence Model (PCM) and goes as follows: consider point particles diffusing on a lattice and carrying an integer which we call their mass. When two particles, of mass $m$ and $m^{\prime}$ respectively, alight on the same site, they combine at a given rate $k$ to form a particle of mass $m+m^{\prime}$. The rate $k$ is often taken to be infinite, that is, the formation of the new particle is instantaneous. The new particle is again pointlike. On the other hand, the rate at which it diffuses may depend on the mass $m$ as an arbitrary function 
$D(m)$, which goes asymptotically as $m^{-\gamma}$.

Note in passing that this model already contains a considerable amount of structure. Its main unrealistic fetaure is that the particles remain pointlike. The great advantage of this assumption is, that it frees us from considering issues of morphology. Indeed, if we assume that clusters connect rigidly when they approach within one lattice spacing of each other, the model becomes much more complex: in particular, it then generates fractal structures, and the radius of the cluster will depend in a highly non-trivial way on the mass. Such a model, simulated in three dimensions, already deserves to be called a realistic model of colloid formation in the Brownian regime. However, since nearly nothing can be said about it at the analytical level, I shall not treat it any further, but refer the interested reader to the extensive literature (see e.g. $[47,78]$ and references therein).

The PCM, introduced by [48] can be treated rigorously as long as the diffusion constant is mass-independent. In this case, see [66] it has been shown that for space dimension $d \geq 3$ the model behaves qualitatively like the mean-field model with a constant kernel. While this may seem encouraging, we shall in fact see that this feature is intimately linked to the pointlike nature of the particles. On the other hand, in two space dimensions, logarithmic corrections appear, whereas in one dimension an explicit exact solution is available $[93,94]$, which displays a number of highly anomalous features.

For arbitrary diffusion constants, on the other hand, no such rigorous treatment is available. Let us shortly argue what mean-field theory we should expect to hold: as is well known, the current which flows into an immobile absorbing sphere of radius $R$ surrounded by a sea of particles diffusing with a diffusion constant $D$ is $D R^{d-2}$ for $d>2$. From this we may plausibly argue that the rate at which one diffusing particle encounters another is proportional to $D_{1}+D_{2}$ as well as to $\left(R_{1}+R_{2}\right)^{d-2}$. Here $R_{i}$ are the radii of the two particles and $D_{i}$ their diffusion constants. Thus, if $R_{i}$ is always the same, we may disregard it and we are eventually left with

$$
K\left(m, m^{\prime}\right)=D(m)+D\left(m^{\prime}\right) .
$$

If the space dimension $d \leq 2$, matters are more complex, since any two particles are sure to react eventually. As we shall see, however, for $d \leq 2$, mean field theory is not expected to work at all, so the precise form of the reaction coefficients is a rather academic issue.

At this point, it may be worthwhile to point out that it is occasionally possible to mimic the results produced by correlations through an appropriate choice of reaction term, different from quadratic. Here I shall systematically disregard such possibilities: when a reaction involves two bodies, I shall take the attitude 
that only a quadratic expression can be viewed as an adequate reaction term describing it.

\subsubsection{Simple Scaling Arguments}

In the following, I shall show how very straightforward arguments of the kind that have been used throughout this paper, can be used to predict the behaviour of the PCM. In the following subsection, I shall briefly present the exact one-dimensional solution due to Spouge [93,94], as well as shortly mention the corresponding rigorous results concerning the two-dimensional model, thus partly confirming the results to be derived now.

First let us derive the growth of the average cluster size. Let us assume that a "typical" particle starts out by being a monomer and grows in such a way as to be typical, that is, of size $s(t)$ all the time. If we consider the probability $P(x, t)$ of finding the particle at $x$ at time $t$ given that it was at the origin at time $t=0$, one has

$$
\begin{aligned}
& \frac{\partial P}{\partial t}=D[s(t)] \Delta P=s(t)^{-\gamma} \Delta P \\
& P(x, 0)=\delta(x) .
\end{aligned}
$$

From this follows that the probability of finding the particle at a distance of order $L$ from the origin is of order one if

$$
L=O(\sqrt{D[s(t)] t})=O\left(t^{(1-z \gamma) / 2}\right) .
$$

Therefore we expect the particle to have covered a space of order $L$ in time $t$. Since originally the total mass in this interval was of order $L$, and it did not change except through diffusion at the boundaries, which can be neglected, we expect the typical size to grow as $L$, or in other words

$$
z=\frac{1}{2+\gamma}
$$

Now let us turn to the values of $\tau$ and $w$. At this (low) level of sophistication, we shall assume that strong scaling holds throughout and shall have no qualms in identifying the decay exponent for monomers with $w$. We may picture the monomer decay problem as follows: each monomer is surrounded by two particles of typical size. These grow normally by coalescing with other particles outside the interval which connects them and which contains the monomer. These particles' growth in no way affects the monomer's survival. The problem therefore reduces to a three particle problem, where the two 
outer particles have a time-dependent diffusion constant, whereas the middle particle diffuses normally. The question is then to determine the decay law for the survival probability of the middle particle. This can then be identified with the monomer concentration decay at large times. This problem is somewhat technical and was studied in [35], with the following result: if $\gamma<0$, that is if the aggregate diffusion constant grows with size, there is a fair probability that the two large particles will diffuse quite far away, letting the middle particle survive for quite long times. The result then is given by

$$
c_{1}(t)=\text { const. } \cdot t^{-2 /(2+\gamma)} \quad(t \rightarrow \infty)
$$

For $\gamma=0$, on the other hand, a straightforward solution is known $[48,27]$ which yields

$$
c_{1}(t)=\text { const. } \cdot t^{-3 / 2} \quad(t \rightarrow \infty)
$$

If we now apply (3.25) to the values of $w$ obtained from (5.5) and (5.6), one obtains

$$
\tau=\left\{\begin{array}{cc}
0 & (\gamma<0) \\
-1 & (\gamma=0)
\end{array}\right.
$$

If we now compare this with the strict mean-field prediction for which we use the kernel (5.1), which is really the only one with a more or less fundamental significance for the PCM, we obtain for the mean-field growth exponent $z_{M F}$

$$
z_{M F}=\frac{1}{1+\gamma}
$$

With respect to $\tau_{M F}$ our knowledge is less complete, since kernel (5.1) is of type II, for which no closed expression for $\tau$ is available. Nevertheless we know from (3.47) that it satisfies the inequality

$$
\tau_{M F} \leq 1-\gamma
$$

Both (5.8) and (5.9) are, of course, incompatible with our scaling theory.

At this stage, one might nonetheless wonder whether some appropriate modification of the equations could possibly describe the system. From the value of $z$ one deduces that the degree of homogeneity $\lambda$ should be equal to $-\gamma / 2$. The fact that $w \neq 1$ excludes a type I kernel, so we are left with the possibility of type II. From the inequality (3.47) we see that $\gamma>\tau$, which is indeed always 
satisfied. But in fact, there is a far more serious objection to such attempts: let us compute the probability of finding a particularly large cluster. This is clearly related to the possibility that any given particle might go particularly far in time $t$. This probability decays as a Gaussian, and not as an exponential. But we have seen (see subsection 3.5.2), that under very general hypotheses, the large size behaviour of a cluster size distribution is always given by an exponential. We see therefore that even in this apparently exceptionally simple case, no simple adaptation of mean-field will give an adequate description of the system's behaviour at large times.

Let us now turn back to the case in which $\gamma>0$. Then the two particles surrounding the monomer diffuse apart at an ever decreasing rate, leading thereby to very rapid trapping of the central monomer. More specifically, one has

$$
c_{1}(t)=\text { const. } \cdot \exp \left[- \text { const. } \cdot t^{\beta}\right] \quad(t \rightarrow \infty)
$$

The determination of $\beta$ involves severe analytical difficulties. Hellén et al. [35] suggest the following approximate value obtained from a Lifshitz tail argument

$$
\beta=\frac{1+\gamma}{4+2 \gamma}
$$

Again this is in qualitative agreement with the Smoluchowski picture, but in clear quantitative disagreement: indeed, for $\gamma>0$, kernel (5.1) is of type III, so that we expect small monomers to decay as stated in (5.10), with $\beta$ given by

$$
\beta=\gamma
$$

in clear contrast to (5.11). The authors in [35] also state unambiguously that even fairly coarse numerical measurements invalidate the mean-field prediction (5.12) quite clearly.

\subsubsection{An Exact Solution}

In the one-dimensional case, if the diffusion constants of the aggregates are size-independent, that is, if $\gamma=0$ in the notation of the previous subsection and if, additionally, reaction between aggregates is instantaneous upon contact, an exact solution was found by Spouge in $[93,94]$. Here I wish to give an outline of the way in which this solution can be obtained, as well as to point out that it does, in fact, confirm the results suggested by the previous, admittedly coarse, scaling analysis. We shall, however, follow a slightly different approach in the spirit of that pioneered by Doering and ben-Avraham [21,22]. 
It is clear, by the definition of the model described above, that we are dealing with a Markov process. This implies that one can write down a master equation for the joint probability distribution function at time $t$ for finding the first particle at $x_{1}$, the second at $x_{2}$, and so on to the $N$-th, where the $x_{k}$ represent the lattice position at time $t$ of the particle $k$. This equation, however, is far too complex to be of any use, and also contains more information than is really needed. The standard approach consists, as is well-known, in taking correlation functions involving one or more particles. In this case, this leads to an intractable set of equations, in which each correlation function couples to one of higher order. If, on the other hand, we consider a different kind of reduced description, which takes the particular one-dimensional nature of the system fully into account, one can indeed find a closed set of equations. This goes as follows: define $E(k, x)$ as the probability of finding a total mass $k$ contained in an interval of length $x$. The crucial simplifying feature about this particular choice of correlation function is, that it remains unchanged by all reactions that take place either inside or outside the relevant interval. In other words, it is only affected by events in which particles move inside or outside the given interval via diffusion. And this, understandably, can be solved.

Let us translate the above verbal arguments into equations. First define $F(k, x)$ to be the probability of having a total mass $k$ inside a given interval of length $x$, and additionally that the leftmost site of this interval be occupied, irrespectively of the state of the rightmost site. In this case, one has

$$
F(k, x)=E(k, x)-E(k, x-a),
$$

where $a$ is the lattice spacing, which also indicates the length which particles jump in one diffusion step.

For simplicity we now restrict ourselves to a continuum limit, in which the particles are far apart on the scale $a$. In this case, we may discard the possibility of both ends of the interval being occupied, so we have for the time variation of $E(k, x)$

$$
\begin{aligned}
\partial_{t} E(k, x) & =F(k, x+a)-F(k, x) \\
& =D \Delta E(k, x)
\end{aligned}
$$

where $D$ denotes the macroscopic diffusion constant arising from the continuum limit. In the derivation of (5.14) we have made essential use of the fact that sites cannot be doubly occupied, which follows from the instantaneous reaction rate. We now need initial and boundary conditions for (5.14). One finds, after some careful considerations involving the original discrete model, that 


$$
\begin{aligned}
& E(k, 0)=0 \\
& \left.\partial_{x} E(k, x)\right|_{x=0}=c_{k}(t)
\end{aligned}
$$

for all times $t$. Furthermore, if the initial particle density is $\rho_{0}$, one has

$$
E(k, x ; t=0)=\frac{\left(\rho_{0} x\right)^{k}}{k !} e^{-\rho_{0} x},
$$

at least, if the monomers are initially distributed at random ${ }^{20}$. It is now straightforward to solve for (5.14) with initial conditions (5.16) and the absorbing boundary condition at the origin (5.15). Using the method of images yields

$$
E(k, x)=\frac{\rho_{0}{ }^{k}}{2 k ! \sqrt{\pi D t}} \int_{0}^{\infty} y^{k} e^{-\rho_{0} y}\left[e^{-(x-y)^{2} /(4 D t)}-e^{-(x+y)^{2} /(4 D t)}\right]
$$

and hence

$$
c_{k}(t)=\frac{\rho_{0}^{-2}}{2 k ! \sqrt{\pi}(D t)^{-3 / 2}} \int_{0}^{\infty} x^{k+1} e^{-x} \exp \left(-\frac{x^{2}}{4 D \rho_{0}^{2} t}\right) d x
$$

Analytically it is not quite straightforward to evaluate this expression, or even to show that it scales. In terms of generating functions, however, this is easy and is shown in Appendix T. The resulting scaling function $\Phi(x)$ is given by

$$
\Phi(x)=\frac{4 x e^{-x^{2}}}{\sqrt{\pi}}
$$

and the typical size indeed grows as $\sqrt{D t}$. We have therefore confirmed that the decay at large $x$ of the scaling function is faster than exponential, thereby precluding any identification with a mean-field model of the type we have been discussing in the body of this paper. We also show rigorously that the $\tau$ and $w$ values are in fact given by the values predicted in the previous subsection, namely -1 and $3 / 2$. Finally, we may note that (5.18) shows that strong scaling holds in this system: indeed, if $t \rightarrow \infty$ in the r.h.s. of (5.18) while $k$ remains fixed, the integral becomes time-independent and one finds

$$
\lim _{t \rightarrow \infty}\left[(D t)^{3 / 2} c_{k}(t)\right]=\frac{\rho_{0}^{-2}(k+1)}{2 \sqrt{\pi}}
$$

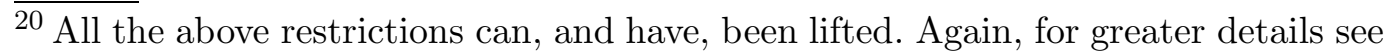
$[93,94]$. 
What have we learned from this example? Primarily we see that in one dimension the mean-field approach fails, essentially because of the buildup of correlations between particles due to the interplay between reaction and diffusion. In particular, one finds that the probability of finding two unreacted aggregates nearby is considerably smaller than expected on the assumption that the reactants are uncorrelated: thus, if we ask for the probability of finding exactly one monomer in each half of an interval of length $x$, that is $E(2, x)-2 E(2, x / 2)$, one finds it to decrease as $x^{3}$ as $x \rightarrow 0$, whereas the square of the probability of finding a monomer in such an interval goes as $x^{2}$. There is, therefore, a strong repulsion between nearby particles, due to the efficiency with which diffusion in one dimension brings nearby particles to react.

It should be pointed out that we have assumed instantaneous reaction rate whenever particles meet. Without this assumption, the model cannot be solved exactly as far as is known. However, it is easy to realize that in the limit where the reaction rate $k$ becomes very small, the transport mechanism becomes irrelevant and mean-field theory holds. However, if particles have an initial separation of order $L$, they will on average meet $L$ times in a time of order $L^{2}$, that is, in a time sufficient for them to come close at all. This follows from well-knnown properties of one-dimensional diffusion. Therefore, if $k L$ is of order one, the problem reduces to the one in which reaction is essentially certain. Since the typical interparticle distance $L(t)$ is growing with time, we should say that when $L\left(t_{c}\right) k$ is of order one, the results obtained above should start to hold, whereas before the description using the constant kernel should be valid. Numerical work does indeed confirm this expectation. The time $t_{c}$ can be evaluated under the assumption that for $t=t_{c}$. $L(t)$ can be evaluated both using the formulae of the constant kernel as those of the diffusive model. One then has

$$
\begin{aligned}
L\left(t_{c}\right) & =\left(\sum_{k=1}^{\infty} c_{k}(t)\right)^{-1}=\text { const. } \cdot k t_{c} \\
& =\text { const. } \cdot\left(D t_{c}\right)^{1 / 2}
\end{aligned}
$$

which implies that $t_{c}$ goes as $D / k^{2}$. One sees therefore that it may be possible to apply the mean-field theory quite meaningfully, even in a system for which we know that it cannot be applied in the true asymptotic regime.

A similar reasoning leads one to conjecture that the mean-field description for the PCM with $\gamma=0$ becomes accurate in dimensions 3 and higher. This is due to the fact that random walks are transient in this case, that is, they visit each point only a finite number of times. One does not expect, therefore, that the correlations created by the diffusion and the reaction process should build up indefinitely in time. Indeed, these expectations have been confirmed: 
specifically, it has been shown (see e.g. [66]) that the cluster size distribution can in this case be scaled onto an exponential distribution, just as for the constant kernel, which is the one we expect to model this particular system.

Finally, I believe this system also shows very nicely the extent to which the concepts originally developed in the context of the mean-field equations can be carried over to more general situations: thus the basic scaling relationship (3.25) connecting $\tau, w$ and $z$ follows from mass conservation alone and holds in all cases. Similarly, the distinction between power-law polydisperse and bell shaped scaling functions also generalizes, as we have seen in the previous subsection.

\subsection{Ballistic Aggregation}

Another simple model of aggregation is the following: consider particles of a given radius $a$ originally spread at random in $\mathcal{R}^{d}$ with random velocities. These then move freely, and stick irreversibly upon any two coming closer to each other than the capture radius $a$. In order to specify the model completely, we require two additional pieces of information: first, how does the radius grow upon irreversible sticking? Here many solutions are in principle possible. We will focus entirely on the two following: either the radius does not grow, or else it grows deterministically in such a way as to maintain the total aggregate volume constant. The second is of course more realistic, but considerably more difficult to discuss, as well as more controversial. Second, we need to know how the velocity of the compound particle is obtained. Since we are physically assuming that this represents sticking of freely moving particles, we shall always assume that momentum conservation determines the final velocity, which it does in a unique way. Since the two particles combine to one inelastically, we have, of course, no conservation of energy.

Let us first consider the one-dimensional case. The two growth rules for the radius are then equivalent. This model was first considered by Carnevale et al. [15] and later investigated further by Jiang and Leyvraz [45] using numerical simulations together with qualitative scaling arguments. We present these first, and later discuss shortly an elegant exact solution [75,29], which confirms the results anticipated in [45] in a very satisfactory way.

\subsubsection{Simple Scaling Arguments}

As we have seen in the case of the diffusive one-dimensional model, it is often a good strategy to search for the long time behaviour of the typical size and the long-time decay of very small clusters, since this contains essentially most of the relevant information. In [15] it was shown that the typical size in one- 
dimension grows as $t^{2 / 3}$ using the following argument: an aggregate of mass $m$ has arisen, in the discrete picture, out of $m$ monomers. These all had momenta of order one, which averaged, as vectors, to zero. If we therefore assume that they are essentially independent, we are led to assume that the momentum $p(m)$ of the $m$-mer is of order $m^{1 / 2}$, which leads to a velocity $v(m)$ of the order of $m^{-1 / 2}$. This leads to a typical aggregate of size $s(t)$ crossing in a time $t$ a length of order $v[s(t)] t$, which is of order $t / \sqrt{s(t)}$. Since the number of particles initially contained in this interval will be of the same order as $s(t)$, we wind up with

$$
s(t)=\text { const. } \cdot\left(\rho_{0} t\right)^{2 / 3},
$$

where $\rho_{0}$ again denotes the initial particle concentration.

This reasoning is quite robust, but does make an assumption concerning the initial velocity distribution function (VDF), namely that it has no large velocity power-law tails. Indeed, the argument which invokes the central limit theorem fails, and $s(t)$ grows in a different way when such tails are present. Indeed, one finds [45] that if the VDF has a distribution with a behaviour of the type

$$
p(v)=\text { const. } \cdot v^{-\alpha-1} \quad(1<\alpha<2)
$$

then one has using an obvious extension of the above reasoning, that the exponent $z$ is given by

$$
z=\frac{\alpha}{2 \alpha-1}
$$

The assumption $\alpha>1$ is necessary, since otherwise the typical velocities would grow on average, making the whole approach questionable. No meaningful numerical results have been obtained for this case, whereas (5.24) has been well confirmed numerically for the range $1<\alpha<2$ [45].

To complete our scaling argument, we should find the asymptotic decay for very small clusters. A completely general lower bound on $c_{1}(t)$ can be derived, quite independently of the initial VDF, in the following way: consider the aggregates at time $t$. It is possible, of course, to identify the positions at time zero of the monomers conforming them. These fall in consecutive intervals of typical length $s(t)$ separated by intervals of length one. If both the following events occur, the initial particle will survive until time $t$,

(1) The particle should first have been placed on one of the empty regions between two of the intervals constituting the two neighbouring typical clusters. This has probability $1 / s(t)$. 
(2) The particle should have been launched with a velocity small enough that it will not reach either of the two neighbouring clusters in time $t$. Since the two clusters are eventually separated by a distance $s(t)$, this limiting velocity is of the order $s(t) / t$. If the VDF has a finite probability density for velocity zero, this leads to a probability of the order $s(t) / t$ as well.

Combining the two probabilities one obtains

$$
c_{1}(t) \geq \text { const. } \cdot t^{-1} \text {. }
$$

It further appears quite unlikely that monomers could survive under any other circumstances than the ones stated above, so that (5.25) is presumably the exact order of magnitude of the monomer decay. Note in passing that we have assumed that the VDF is finite at zero velocities. What happens, for example, if all initial velocities are taken with values \pm 1 ? At first sight, this changes a lot, since odd clusters always have a lower (mass-dependent) bound on their velocities, whereas even ones always have a finite probability of having velocity exactly zero. Therefore, for a fixed even value of the mass one has $c_{2 j}(t)$ going asymptotically as $1 / s(t)$, which differs from (5.25). Similarly, for fixed odd values of the mass, we get exponential decay of $c_{2 j+1}(t)$ for large times. However, it is easy to see that in the scaling limit the continuum approximations can in fact be made, so that the scaling exponent $w$ is 1 in this case also.

From this evaluation of $w$ follows via (3.25) that

$$
\tau=\left\{\begin{array}{cc}
1 / \alpha & (1<\alpha<2) \\
\frac{1}{2} & (\alpha>2),
\end{array}\right.
$$

where the usual case is contained in the second. Numerically, these claims have been verified ${ }^{21}$ in [45].

Now let us compare with mean-field theory. To this end, we need to develop a plausible expression for the reaction rates $K\left(m, m^{\prime}\right)$. A common and frequently used one for related problems (see e,g, [23]), is the following:

$$
K\left(m, m^{\prime}\right)=\left|v(m)-v\left(m^{\prime}\right)\right|\left[R(m)+R\left(m^{\prime}\right)\right]^{d-1},
$$

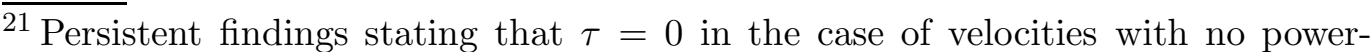
law tail have repeatedly appeared in the literature. Since they are now found to be contrary to exact results $[75,29]$, it may be enough to say here that they are erroneous. 
where $v(m)$ is the velocity of a typical aggregate of mass $m$ and $R(m)$ its radius. Using the standard approach outlined in this paper, one finds for the growth exponent $z$ in the case where $v(m)$ goes as $m^{-1 / 2}$ and $R(m)$ constant, that $z$ is in fact $2 / 3$ as expected. On the other hand, in this case, the kernel (5.18) is of type III, so that the monomer concentration $c_{1}(t)$ should go as a stretched exponential, specifically

$$
c_{1}(t)=\text { const. } \cdot \exp \left(- \text { const. } \cdot t^{1 / 3}\right)
$$

It is, of course. quite evident what is going wrong here: in (5.27) we are assuming that monomers always react as if they were moving at a velocity of order one, whereas it is clear that the velocity of the surviving monomers is a decreasing function of time, due to fast monomers being selectively eliminated by the reaction. In fact, it is seen numerically that the velocity profile $v(m)$ does not decay with mass, but is on the contrary rather flat, decaying as $s(t)^{1 / 2}$ with time. There is also (somewhat inconclusive) evidence [45] that (5.28) actually holds for very small reaction rates, so one may in fact argue that one is using the "correct" mean-field theory.

A more sophisticated approach involves considering the joint distribution of mass and momentum. In this case we may view the vector $(m, p)$ as a vector valued mass, in a way similar to the one in which we treated multicomponent aggregation. One then finds for the reaction kernel

$$
K\left(m, p ; m^{\prime}, p^{\prime}\right)=\left|\frac{m}{p}-\frac{m^{\prime}}{p^{\prime}}\right|\left[R(m)-R\left(m^{\prime}\right)\right]^{d-1} .
$$

Since the $p$ are on average zero, we are in the same situation as when discussing the scaling of composition. However, it is easy to see that, at least within the treatment of composition scaling that we have given, the behaviour of small clusters is essentially the same. The gaussian behaviour of the momenta is justified in detail by our approach. On the other hand, it should be admitted that we have not shown that these are the only possible solutions to the multicomponent scaling equations (3.140).

\subsubsection{Exact Results}

Here I shortly review the exact solution derived in $[75,28,29,30]$. The precise form of the solution is quite formidable and its derivation is an impressive feat indeed. I shall not, however, go into the details, which are rather technical, and for which the interested reader is referred to the original literature.

In order to state the result in a readily understandable form, let me define some notation. I denote by $\mathcal{T}\left(x_{1} . y_{1} ; x_{2}, y_{2}\right)$ the following probability: Let $y(x)$ 
be a random function of $x$ chosen according to the Wiener measure, or, in other words, a Brownian motion directed along the $x$ axis. $\mathcal{T}$ is then the probability that it satisfy

$$
\begin{aligned}
& y\left(x_{1}\right)=y_{1} \\
& y\left(x_{2}\right)=y_{2} \\
& y(x)<x^{2} \quad\left(x_{1}<x<x_{2}\right)
\end{aligned}
$$

In other words, it is the probability that a directed random walk start at $\left(x_{1}, y_{1}\right)$ and reach $\left(x_{2}, y_{2}\right)$ without having ever touched the "absorbing parabola" $y=x^{2}$. This can also be descibed as the solution to the following partial differential equation

$$
\frac{\partial \mathcal{T}\left(x_{1}, y_{1} ; x, y\right)}{\partial x}=\frac{\partial^{2} \mathcal{T}\left(x_{1}, y_{1} ; x, y\right)}{\partial y^{2}}
$$

with the boundary conditions

$$
\begin{aligned}
& \mathcal{T}\left(x_{1}, y_{1} ; x_{1}, y\right)=\delta\left(y-y_{1}\right) \\
& \mathcal{T}\left(x_{1}, y_{1} ; x, x^{2}\right)=0
\end{aligned}
$$

From this one now defines a few auxiliary functions: first I introduce $\mathcal{T}_{\infty}\left(x_{1}, y_{1}\right)$, which is the probability of reaching infinite values of $x$ without having been absorbed by the parabola. This is given by

$$
\mathcal{T}_{\infty}\left(x_{1}, y_{1}\right)=\lim _{x \rightarrow \infty} \int_{-\infty}^{x^{2}} \mathcal{T}\left(x_{1}, y_{1} ; x, y^{\prime}\right) d y^{\prime}
$$

Finally, we shall be interested in the relative probabilities of escape of particles that start very near to the parabola. To this end we define

$$
\begin{aligned}
I\left(x_{1}, x_{2}\right) & =\left.\frac{\partial^{2}}{\partial y_{1} \partial y_{2}} \mathcal{T}\left(x_{1}, y_{1} ; x_{2}, y_{2}\right)\right|_{y_{1}=x_{1}^{2} ; y_{2}=x_{2}^{2}} \\
J\left(x_{1}\right) & =\left.\frac{\partial}{\partial y_{1}} \mathcal{T}_{\infty}\left(x_{1}, y_{1}\right)\right|_{y_{1}=x_{1}^{2}}
\end{aligned}
$$

The final result can now be stated as follows: the concentrations $c(m, v, t)$ obey the following scaling law

$$
c(m, v, t)=t^{-1} \Phi(x, \eta) .
$$


Here $x$ and $\eta$ represent the following scaling variables

$$
\begin{aligned}
& x=\frac{m}{(2 \sigma t)^{2 / 3}} \\
& \eta=(2 \sigma t)^{1 / 3} v,
\end{aligned}
$$

where $\sigma$ is the r.m.s. velocity in the initial VDF. One then finds for $\Phi(x, \eta)$

$$
\Phi(x, \eta)=I\left(-\frac{x}{2}, \frac{x}{2}\right) J\left(\frac{\sigma x-\eta}{2 \sigma}\right) J\left(\frac{\sigma x+\eta}{2 \sigma}\right)
$$

Several points should be noted about this result: first, the mass and velocity dependence do not factorize, nor do they show the simple kind of interdependence we have found in multicomponent aggregation. In fact, it is explicitly shown in [28] that the collision probability between two aggregates does not factorize. In this way, it is clear that any attempt to build a mean-field theory is ill-founded, and indeed such attempts have failed to reproduce known results.

The derivation of this exact solution is very interesting in its basic ideas, but quite laborious in the details. In Appendix U, I therefore attempt to present the concepts to the extent that a sufficiently enthusiastic reader should be able to fill in the missing algebra.

One can then use (5.31) to evaluate $\mathcal{T}$, and hence all other functions defined above, in terms of Airy functions. This opens the way to an asymptotic analysis of $\Phi(x, \eta)$, and of its integral over $\eta$. This is a laborious undertaking, which was performed in the above references, where they confirm the scaling results stated in [45]. Additionally, an unexpected large $x$ asymptotic is obtained, namely

$$
\int_{-\infty}^{\infty} \Phi(x, \eta) d \eta=\text { const. } \cdot \exp \left(-x^{3} / 12\right) \quad(x \gg 1)
$$

However, it is not clear whether this might not be an artefact of initial conditions in which all particles are put on a lattice: it seems incredible that normal density fluctuations when particles are put at random on the line should still satisfy (5.40). In any case, however, this large $x$ behaviour once more shows how impossible it is to treat this model in mean-field terms.

\subsubsection{Higher Dimensions}

The case of higher dimensions is both far more complex and, to some extent, controversial. First, it is obvious that it is important, in this case, to 
specify how the particle radii grow upon aggregation. We shall consider two possibilities:

(1) The particle radius remains fixed when the particles stick together. This is obviously unrealistic, but it has the merit of greater simplicity. In particular, there is a real hope that mean-field theory might hold in $d \geq 2$.

(2) The particle radius grows so as to maintain the total $d$ dimensional aggregate volume constant. Since the total occupied volume fraction remains always finite, this is a really difficult model. Thus, even three-particle collisions cannot be discarded out of hand as being asymptotically irrelevant. I shall have little to say on that count.

Consider first the case of fixed radius. We may then attempt to formulate once more the mean-field theory. In this case expression (5.27) for the reaction kernel still holds, and leads to

$$
z=\frac{2}{3}
$$

independently of $d$. The monomer decay predicted by (5.28), namely a stretched exponential with exponent $1 / 3$, still holds independently of $d$. This is in fact quite natural, since the reaction kernel defined by (5.27) only depends on $d$ via the geometric cross-section, which we have assumed constant. In this sense, kernel (5.27) is much more general than the previous analysis indicated.

Is this mean-field theory likely to be correct? Since the total number concentration decays as $t^{-2 / 3}$, the mean free path grows as $t^{2(d-1) / 3}$, which is always more than the interparticle distance $t^{2 /(3 d)}$ when $d>1$. This is therefore extremely encouraging, since it shows that typically colliding particles will be distant on the scale of nearest neighbour separation. In related work it has been shown that the Boltzmann equation becomes exact at large times [85] for the model of $d$ dimensional ballistic annihilation when $d>1$. A numerical confirmation of all this would be of considerable interest, but has not, to the best of my knowledge, been performed to date.

The model in which the radii grow as $m^{1 / d}$, on the other hand, is far more complex. The obvious mean-field theory has again a reaction kernel of the form (5.27), which has values of $\lambda$ and $\mu$ given by

$$
\begin{aligned}
& \lambda=\frac{d-2}{2 d} \\
& \mu=-\frac{1}{2}
\end{aligned}
$$

from which one deduces in the ordinary way that 


$$
\begin{aligned}
z & =\frac{2 d}{d+2} \\
c_{1}(t) & =\text { const. } \cdot \exp \left[- \text { const. } \cdot t^{d /(d+2)}\right]
\end{aligned}
$$

There is therefore again a stretched exponential decay of the monomers according to this theory. This is again due to the fact that we do not take into account the selective elimination of high velocity monomers in this model. But since, in this model, the mean free path of a monomer always remains of the order of the interparticle distance (due to the conservation of the occupied volume fraction), it appears very likely that such an elimination will in fact take place.

Trizac and Hansen have performed some high quality numerical simulation on the two dimensional version of this model [98]. These lead apparently to predictions quite at variance with the above theory. However, it should be borne in mind that the data were never explicitly analyzed in terms of the theory described here, but rather compared with a rather peculiar "meanfield" theory [84], which includes, however, some correlation effects by means of various uncontrolled approximations. This theory's principal merit was to provide a rationale for the observation of a "pure exponential" cluster size distribution, together with $\tau=0$, which was then thought to be the correct result in one dimension [15]. The agreement of the two-dimensional simulations performed in [98] with the theory presented here is therefore rather difficult to assess.

Nevertheless, let us us shortly discuss the most salient differences between the simulation [98] and theory. The measurement of the $z$ exponent ${ }^{22}$ yields a result which is claimed to be significantly different from the theoretical value of one. The figure published does not, however, show a very convincing straight line behaviour over any range of sizes. The best that can be said is that strong crossover effects are certainly present, which make it difficult to say with certainty what the actual value of $z$ is. The quoted value of 0.8 (for the dilute system, which is the one of greater theoretical interest, as well as the one for which deviations from theory are largest) is really quite far from the expected value, however, so I feel strongly that a more careful analysis of the data is required before any definite statement concerning the validity of mean-field theory can be made.

No measurements either of the monomer decay or of the $\tau$ exponent were made. It is therefore not possible to decide the crucial issue whether the stretched exponential decay predicted by (5.44) indeed takes place. The approximately exponential appearance of the cluster size distribution does, however, make it highly unlikely.

$\overline{22 \text { called }} \xi$ in [98] 
On the other hand, the average kinetic energy per particle was measured and yielded a power law decay $t^{-\delta}$ incompatible with the prediction of mean-field theory. Indeed, from the mean-field arguments given above one obtains

$$
\delta=z
$$

whereas the quoted value for the dilute case is 1.12 . This is certainly a serious disagreement with the measured value of $z$. If we take this result seriously, it suggests that the cancellation between velocities of colliding particles leads to velocities far less than what would be implied by the Central Limit Theorem. The intriguing suggestion that this might be due to the kinematics of collision was made in [99]. A considerable amount of work clearly remains to be done to settle these important issues.

\section{Conclusions and Outlook}

I have attempted to give a comprehensive overview of the way in which scaling concepts can be used in the context of the kinetics of irerversible aggregation. We have seen that applications are numerous and the method is quite powerful in predicting, among others, various qualitative behaviours both for the shape of the cluster size distribution and for the time dependence of the rescaling factors involved, such as the typical size or length. There remain nevertheless several shortcomings to the method, which might perhaps be overcome and lead to a technique that could be more quantitative even for fairly complicated systems. Here are some aspects which I believe to be of interest.

- Once one has an integral equation for the scaling function $\Phi(x)$, difficulties are by no means over. It is then necessary, in practical applications, to solve it, generally by some numerical approach. This turns out to be a difficult problem. The various forms into which the basic equation (3.12) can be cast, due to the presence of an arbitrary function $f(x)$, might possibly be of use. On the other hand, for numerical work, the original form of the equation as given in (3.35) is presumably optimal, since it contains the desired function in the r.h.s. of the equation, whereas the corresponding relations involving exponentials yield objects such as the Laplace transform of $\Phi(x)$, which is definitely less direct.

The issue of computing scaling functions becomes particularly acute in cases such as crossover or inhomogeneous systems, for which the function

$\Phi(x, y)$ has two variables and the integral equation is complicated. In these cases, even the behaviour of the function at the origin is not straightforward. In this respect, it would be of interest to have a theory of comparable simplicity as that of regular variation for functions of two or more variables. 
It is not clear that this is an attainable goal, however, as functions in two variables can exhibit a strikingly complex set of singular behaviours.

- I believe crossover may be of considerable use in practical applications, since a competition of two reaction mechanisms is a frequent occurence. The progress reported here, while possibly of interest, is certainly only a first step: more exactly solved models exhibiting crossover should be looked into and those for which I have not been able to give solutions, such as the $q$ sum kernel in the limit $q \rightarrow 1$, should be viewed as relevant problems. Further, the integral equation has almost yielded no results of interest on the form of the two-variable scaling function $\Phi(x, y)$. To obtain a theory yielding the form of the singularity at least in some limiting cases is of crucial importance. It must be admitted, however, that the existing exact solutions do not hold out much hope for broad ranges of simple behaviour.

- Exact solutions for models with production and diffusion are, to the best of my knowledge, absent except for some work relying on the simplifying features arising from stationarity. I believe any such model would be of interest, since we have shown that the stationary limit in this case does not arise in an easy way from the non-stationary case.

- Are there cases in which the scaling function $\Phi(x)$ can be obtained exactly, though the kernel itself be not solvable? This would increase considerably our understanding of aggregation, in particular if we could obtain rigorous results for some type of kernel of type III or type I, for which, so far, no analytical results are known.

- Can one go any further than I have done towards elucidating the singularity of the scaling function $\Phi(x)$ for the gelling case? It should be possible to obtain some kind of estimates on $\tau$, perhaps by using methods similar to those developed by Cueille and Sire for Case II kernels [18,19]. From the mathematical viewpoint this is certainly important. On the other hand, from the point of view of applications, it may well be enough to compute such exponents to sufficient accuracy. To this end, however, it would again be essential to have a good way to solve the equation, in spite of the appearance of the unknown exponent $\tau$ in it.

- There also remains a certain mystery around the Case I non-gelling kernels. These are quite important, yet there remains some doubt concerning the very existence of a scaling function for this case. The numerical work of Lee [56] certainly makes a very strong case for existence, but numerical evidence, of course, is never entirely conclusive on its own. This work seems to point to a highly anomalous patterns of corrections to the leading powerlaw behaviour, that are oscillatory and might, in point of fact, violate the hypothesis of regular behaviour near the origin. A different mathematical framework should be designed in order to derive such results as are strongly suggested by the numerics.

- Finally, I believe corrections to scaling may be of interest: by this I do not mean the corrections to the leading power-law of the scaling function: this has already been done, at least for Case II in [108]. There is, however, an- 
other issue, hinted at in [7], namely that scaling behaviour is not reached immediately. Thus, initial conditions may create broad transients, which should also be treated at least to some approximation. In [7] the consequences of having an initial condition containing both monomer and $N$-mer are worked out explicitly. More work along this direction would be desirable, as this is not at all explored until now.

There also remain several mathematical problems related to the theory of the

Smoluchowski equations. First and foremost, I would mention the necessity of obtaining optimal results for the system of equations describing coupled diffusion and aggregation. I believe present results, which only concern diffusion constants bounded from below for large masses, are probably not as strong as they could be made. Finally, several old problems are still there: proving the scaling hypothesis under fairly general hypotheses is one. An issue which may also be related concerns the relation between the stochastic models of aggregation and the deterministic Smoluchowski equations. In the framework of van Kampen's $\Omega$ expansion, very remarkable results have in fact been obtained. A corresponding rigorous result was proved in [81], which may well pave the way to future progress.

\section{Acknowledgements}

I would first like to thank Itamar Procaccia for encouraging me to write this article. I have also greatly benefitted from helpful conversations with F. Calogero, P.L. Krapivski, H. Larralde, S. Redner and E. Trizac. The financial support of DGAPA project IN112200 and CONACyT 32173-E is also gratefully acknowledged.

\section{A Derivation of the Scaling Equation for $\Phi(x)$}

The fact that the $c(m, t)$ approach a scaling form, as stated in (3.12), can be reformulated as follows:

$$
\lim _{t_{1}, t_{2} \rightarrow \infty} \int_{t_{1}}^{t_{2}} \frac{d}{d t}\left[\int_{0}^{\infty} m f[m / s(t)] c(m, t)\right]=0
$$

We now rewrite the integrand using (2.2) as follows

$$
\frac{s(t)^{-1}}{2} \int d m_{1} d m_{2} m_{1} m_{2} c\left(m_{1}, t\right) c\left(m_{2}, t\right) K\left(m_{1}, m_{2}\right) G\left[m_{1} / s(t), m_{2} / s(t)\right]-
$$




$$
-\frac{\dot{s}(t)}{s(t)} \int d m m \frac{m}{s(t)} f^{\prime}[m / s(t)] .
$$

Here $G(x, y)$ is given by

$$
G(x, y)=\frac{1}{x y}[(x+y) f(x+y)-x f(x)-y f(y)]
$$

If we now assume that we can replace $K\left(m_{1}, m_{2}\right)$ by its continuous limiting form $s(t)^{\lambda} \bar{K}\left[m_{1} / s(t), m_{2} / s(t)\right]$ - something we shall justify shortly - then (A.2) is of the same form as the l.h.s of (3.12), since $G(x, y)$ is bounded whenever $f(x)$ is bounded and differentiable. It therefore follows that (A.2) can be recast as

$$
\begin{aligned}
& \frac{W^{2} s(t)^{\lambda-1}}{2} \int_{0}^{\infty} d x d y \bar{K}(x, y) \Phi(x) \Phi(y)[(x+y) f(x+y)-x f(x)-y f(y)]- \\
& -W \frac{\dot{s}(t)}{s(t)} \int_{0}^{\infty} d x x^{2} f^{\prime}(x) \Phi(x) .
\end{aligned}
$$

If this, integrated over $t$, should give zero, as stated in (A.1), then surely one must have

$$
\dot{s}(t)=W_{1} s^{\lambda}
$$

for some constant $W_{1}$. This allows, in principle, to determine $s(t)$ completely, including the prefactor. We find

$$
s(t)=\left[(1-\lambda) W_{1} t+s(0)^{1-\lambda}\right]^{1 /(1-\lambda)}
$$

Note that some difficulties arise when $\lambda=1$, and that the whole scheme becomes inconsistent when $\lambda>1$. The former difficulties are somewhat technical and will be treated in another part of this paper. The second involve, as we shall see, convergence to a scaling form of the second moment rather than the first, and therefore require different considerations.

The determination of $W_{1}$ rests on issues which strongly depend on conventions and personal preferences, such as the exact way in which typical size is defined. We therefore assume in the following that $W_{1}$ has been somehow determined, and proceed to set the constant $W$, which we had previously left free, equal to $W_{1}$. One can then take $W^{2}$ out of the whole expression in (A.4). One further deduces, from (A.1), that (A.4) must vanish, yielding (3.29) after observing that 


$$
\begin{aligned}
G(x, y) & =\frac{1}{y}[f(x+y)-f(x)]+\frac{1}{x}[f(x+y)-f(y)] \\
& =G_{1}(x, y)+G_{1}(y, x) .
\end{aligned}
$$

Since the rest of the integral is symmetric, we may limit ourselves, up to a factor of two, to integrating over $G_{1}(x, y)$.

The one point that is left concerns the justification of substituting $K\left(m_{1}, m_{2}\right)$ by

$$
s(t)^{-\lambda} \bar{K}\left[m_{1} / s(t), m_{2} / s(t)\right]
$$

Let us first consider the case in which $f(x)$ vanishes identically on some interval of the form $[0, \epsilon]$. In this case, the statement is obvious: as $s(t) \rightarrow \infty$, the masses summed over in A.2) become increasingly large, and the convergence of $K$ to $\bar{K}$ as stated in (3.30) is enough to justify the substitution.

We have therefore shown, that (3.29) must hold for all functions $f(x)$ which vanish on an interval near the origin. The general statement is an immediate consequence of this together with the monotone convergence theorem.

\section{B Derivation of the Scaling Equation in the Three-Body Case}

From the point of view of the algebraic manipulations involved, the threebody case is wholly similar to the corresponding two-body computation, and the reader is referred to appendix A for notation and the basic computations. The issue we shall be addressing here concerns not so much the nature of the manipulations involved, but their admissibility.

Up to (A.2,A.3), everything is strictly analogous to the case of appendix A. $G$ is now a function of three variables given by

$$
G(x, y, z)=\frac{(x+y+z) f(x+y+z)-x f(x)-y f(y)-z f(z)}{2 x y z} .
$$

The crucial difference between the two and three body cases now apppears in the following fact: $G(x, y, z)$ is not a bounded function, no matter how $f(x)$ is chosen, unless it be a constant. Indeed, choose $x$ and $y$ so that

$$
(x+y) f(x+y) \neq x f(x)+y f(y) .
$$

It then immediately follows that $G(x, y, z) \rightarrow \infty$ as $z \rightarrow 0$. The only possibility 
left is, therefore, to assume that things will go well. If we then go through the computations that correspond to those given in appendix A, we obtain

$$
\dot{s}(t)=W_{1} s^{\lambda_{3}-1},
$$

which is the result stated in (3.125). We may then, as in Appendix A, set $W_{1}=$ $W^{2}$ and therefore obtain the result stated in (3.125). There is, however, no guarantee that this derivation goes through. It is, in particular, quite possible that the integral

$$
\int d x d y d z x y z K(x, y, z) G(x, y, z) \Phi(x) \Phi(y) \Phi(z)
$$

diverges near the origin. This then indicates, from a physical viewpoint, that processes in which one very small particle coalesces with two particles of typical (but not equal) sizes affect the scaling of the aggregation process in a singular way. I shall not attempt to make a scaling theory of such cases, merely limiting myself to stating when the results obtained by the above theory are self-consistent, that is, when the above mentioned divergences near the origin do not occur. It should be clear that all the results discussed in this appendix carry over straightforwardly to the general case of a reaction of order $r \geq 3$.

We shall see in subsection 4.8 that various exactly solved cases fall under this category. Further, we shall see that in this case, it is not always possible to neglect a two-body contribution which shoud be negligible on naive grounds. Thus, the kernel $m_{1}+m_{2}+m_{3}$ has a naive growth exponent $z=1$. However, the real growth exponent in the pure three-body case is $z=2$. Nevertheless, if we perturb the constant kernel, which also has $z=1$, using this three-body kernel, the combined process has a growth exponent of $z=1$, that is, the expected dominance of the model with the higher value of $z$ does not take place. For larger values of the perturbation, on the other hand, the exponents vary continuously.

\section{The Large- $\rho$ Behaviour of $I(\rho)$}

In the following, we shall always assume that $\Phi(x)$ has regular behaviour near the origin in the sense of (3.41) and that the corresponding power law is given by $\tau$. We now divide $I(\rho)$ in two parts:

$$
I_{1}=\int_{0}^{\infty} d x \int_{0}^{C / \rho} d y \bar{K}(x, y) \Phi(x) \Phi(y) x e^{-\rho x}\left[1-e^{-\rho y}\right]
$$




$$
I_{2}=\int_{0}^{\infty} d x \int_{C / \rho}^{\infty} d y \bar{K}(x, y) \Phi(x) \Phi(y) x e^{-\rho x}\left[1-e^{-\rho y}\right]
$$

I now treat $I_{1}$ and $I_{2}$ separately. For $I_{1}$, a change of variables yields

$$
I_{1}=\rho^{-(\lambda+3)} \Phi\left(\frac{1}{\rho}\right)^{2} \int_{0}^{\infty} d x x^{1-\tau} e^{-x} \int_{0}^{C} d y \bar{K}(x, y) y^{-\tau}\left[1-e^{-y} .\right]
$$

Here the substitution of $\Phi(y / \rho) / \Phi(1 / \rho)$ by $y^{-\tau}$ is justified by the fact that the range of integration of $y$ is finite. A similar justification for $x$ exists always, that is, both for $I_{1}$ and $I_{2}$. Should this yield divergent integrals, one would have to conclude that the regularity assumption was not self-consistent.

For $I_{2}$, we only rescale $x$ by $\rho$ and obtain using (3.2) and (3.3)

$$
I_{2}=\rho^{-2} \Phi\left(\frac{1}{\rho}\right) \int_{0}^{\infty} d x x^{1-\tau} e^{-x} \int_{C / \rho}^{\infty} d y y^{\lambda} k\left(\frac{1}{\rho y}\right) \Phi(y) .
$$

Assuming further that $k(z)$ behaves regularly (in the sense of (3.41) again) with exponent $\mu$ (see (3.4)) one finds the final expression

$$
I_{2}=\rho^{-2} \Phi\left(\frac{1}{\rho}\right) k\left(\frac{1}{\rho}\right) \int_{0}^{\infty} d x x^{1-\tau} e^{-x} \int_{C / \rho}^{\infty} d y y^{\lambda-\mu} \Phi(y) .
$$

Now let us consider the issue of convergence of these various integrals. It is obvious by the above calculation that the sum $I_{1}+I_{2}$ cannot depend on the constant $C$. One sees, moreover, that the integral appearing in the expression for $I_{1}$ converges exactly when the one appearing in $I_{2}$ diverges. The limit separating these cases is

$$
1+\lambda-\mu-\tau=0
$$

Whichever integral diverges is therefore compensated by the correction to scaling generated by the finite cutoff in the converging integral. The expression containing the convergent integral must therefore always dominate. We may therefore write

$$
I=B_{1} \rho^{-(\lambda+3)} \Phi\left(\frac{1}{\rho}\right)^{2}+B_{2} \rho^{-2} \Phi\left(\frac{1}{\rho}\right) k\left(\frac{1}{\rho}\right),
$$

where $B_{1}$ and $B_{2}$ are given by 


$$
\begin{aligned}
& B_{1}=\int_{0}^{\infty} d x x^{1-\tau} e^{-x} \int_{0}^{\infty} d y \bar{K}(x, y) y^{-\tau}\left[1-e^{-y} \cdot\right] \\
& B_{2}=\int_{0}^{\infty} d x x^{1-\tau} e^{-x} \int_{0}^{\infty} d y y^{\lambda-\mu} \Phi(y)=\Gamma(2-\tau) \int_{0}^{\infty} d y y^{\lambda-\mu} \Phi(y) .
\end{aligned}
$$

(C.6) must be here understood in the sense that only the term for which the integral in the corresponding prefactor converges should be taken into account.

Finally, let us consider the case in which $\Phi(x)$ decays faster than any power near the origin. In this case, one can neglect the contribution due to $e^{-\rho y}$ in the expression for $I(\rho)$ given in (3.34). It can therefore be rewritten as

$$
\int_{0}^{\infty} d x x^{1+\lambda} \Phi(x) e^{-\rho x} \int_{0}^{\infty} d y k\left(\frac{y}{x}\right) \Phi(y) .
$$

But one has $y \gg x$ always, since $x$ must be of order $1 / \rho$ and $y$ cannot be small, due to the behaviour of $\Phi(y)$ at the origin. One can thus use the following approximation for $k(z)$

$$
k(z)=\Gamma z^{\nu}[1+o(1)] \quad(z \rightarrow \infty)
$$

from which the result stated in the text readily follows.

\section{The Large- $\rho$ Behaviour for the Three-body Reaction}

One follows exactly the same strategy as in appendix $\mathrm{C}$ to evaluate the various integrals involved. The result is, with exactly the same notations, and the various homogeneity exponents defined as in (3.127):

$$
\begin{aligned}
& B_{1}=\int_{0}^{\infty} d x \int_{0}^{C} d y d z x^{1-\tau} e^{-x} y^{-\tau} z^{-\tau} K(x, y, z)\left[1-e^{-(y+z)}\right] \\
& B_{2}=2 \int_{0}^{\infty} d x x^{1-\tau+\lambda_{2}} e^{-x}\left(\int_{0}^{C} d y y^{-\tau} k_{1}(y / x)\right)\left(\int_{C / \rho}^{\infty} d z z^{\lambda_{3}-\lambda_{2}} \Phi(z)\right) \\
& B_{3}=\int_{0}^{\infty} d x x^{1-\tau} e^{-x} \int_{C / \rho}^{\infty} d y d z z^{\lambda_{2}-\mu} \Gamma_{1}(y / z) \Phi(y) \Phi(z)
\end{aligned}
$$




\section{E The Distribution of Compositions in Multicomponent Aggrega- tion}

In this appendix we show how the compositions scale in the multicomponent case. To this end we require first a scaling equation of the same type as (3.29) or (3.140) for the function $\Psi(\sigma, \delta)$. Again, we use the same approach as in Appendix A and obtain from (3.137)

$$
\begin{aligned}
& \int_{0}^{\infty} d \sigma \int_{-\infty}^{\infty} d \delta \delta\left[\sigma f_{\sigma}(\sigma, \delta)+\alpha \delta f_{\delta}\right] \Psi(\sigma, \delta)= \\
& \quad \int_{0}^{\infty} d \sigma_{1} d \sigma_{2} \int_{-\infty}^{\infty} d \delta_{1} d \delta_{2} K\left(\sigma_{1}, \sigma_{2}\right) \Psi\left(\sigma_{1}, \delta_{1}\right) \Psi\left(\sigma_{2}, \delta_{2}\right) \times \\
& \quad \times\left[\left(\delta_{1}+\delta_{2}\right) f\left(\sigma_{1}+\sigma_{2}, \delta_{1}+\delta_{2}\right)-\delta_{1} f\left(\sigma_{1}, \delta_{1}\right)-\delta_{2} f\left(\sigma_{2}, \delta_{2}\right)\right] .
\end{aligned}
$$

I now substitute for $f$

$$
f(\sigma, \delta)=\frac{\delta}{s^{\alpha}} \exp \left(-\rho \sigma-i q \delta / \sigma^{\alpha}\right) .
$$

I now take (3.141) as an ansatz to be substituted into (E.1) in order to determine the unknown functions $\Phi_{1}$ and $\chi_{\alpha}$. This leads to the equation

$$
\begin{aligned}
& -\rho \int_{0}^{\infty} d \sigma \sigma^{\alpha+1} \Phi_{1}(\sigma) e^{-\rho \sigma} \int_{-\infty}^{\infty} d \delta \chi_{\alpha}(\delta) e^{-i q \delta}= \\
& \quad \int_{0}^{\infty} d \sigma_{1} d \sigma_{2} \int_{-\infty}^{\infty} d \delta_{1} d \delta_{2} K\left(\sigma_{1}, \sigma_{2}\right) \Phi_{1}\left(\sigma_{1}\right) \Phi\left(\sigma_{2}\right) \chi_{\alpha}\left(\delta_{1}\right) \chi_{\alpha}\left(\delta_{2}\right) \times \\
& \quad \times\left[\left(\sigma_{1}+\sigma_{2}\right)^{\alpha} e^{-\rho\left(\sigma_{1}+\sigma_{2}\right)} \exp \left(-i q \frac{\delta_{1} \sigma_{1}^{\alpha}+\delta_{2} \sigma_{2}^{\alpha}}{\left(\sigma_{1}+\sigma_{2}\right)^{\alpha}}\right)-\right. \\
& \left.\quad-\sigma_{1}^{\alpha} e^{-\rho \sigma_{1}-i q \delta_{1}}-\sigma_{2}^{\alpha} e^{-\rho \sigma_{2}-i q \delta_{2}}\right] .
\end{aligned}
$$

For this equation to hold, we need that the r.h.s. show a factorization in the dependence on $q$, since the l.h.s. does. For this to be the case, we need

$$
\int_{-\infty}^{\infty} d \delta_{1} d \delta_{2} \chi_{\alpha}\left(\delta_{1}\right) \chi_{\alpha}\left(\delta_{2}\right) \exp \left(-i q \frac{\delta_{1} \sigma_{1}^{\alpha}+\delta_{2} \sigma_{2}^{\alpha}}{\left(\sigma_{1}+\sigma_{2}\right)^{\alpha}}\right)=\int_{-\infty}^{\infty} d \delta \chi_{\alpha}(\delta) e^{-i q \delta}
$$

As is readily seen, however, this equation is satisfied by the stable symmetric

Lévy distributions and by these only. Indeed, the Fourier transform $\hat{\chi}_{\alpha}(q)$ of 
$\chi_{\alpha}(\delta)$ must satisfy the functional equation

$$
\hat{\chi}_{\alpha}(q)=\hat{\chi}_{\alpha}\left(\frac{\sigma_{1}^{\alpha}}{\left(\sigma_{1}+\sigma_{2}\right)^{\alpha}} q\right) \hat{\chi}_{\alpha}\left(\frac{\sigma_{2}^{\alpha}}{\left(\sigma_{1}+\sigma_{2}\right)^{\alpha}} q\right),
$$

for which the only solution is $\exp \left(-|q|^{1 / \alpha}\right)$.

\section{F The Integral Equation for the Gelling Case}

In this Appendix we derive the basic equation to be satisfied by the scaling function $\Phi(x)$ when convergence to scaling occurs in the sense of the second moment only, as is, for example, characteristic of the situation in which gelation takes place. We follow exactly the same steps as in Appendix A and refer the reader to this Appendix for more detailed explanations.

The definition we shall use of convergence to scaling is the one given in (3.64). In order to ontain the equation for $\Phi(x)$ we shall again start from an obvious consequence, namely

$$
\lim _{t_{1}, t_{2} \rightarrow \infty} \int_{t_{1}}^{t_{2}} I\left(t^{\prime}\right)=0
$$

where we have defined

$$
I(t)=\frac{d}{d t}\left[M_{2}(t)^{-1} \int_{0}^{\infty} d m c(m, t) f\left(\frac{m}{s(t)}\right)\right]
$$

Let us now evaluate $I(t)$ :

$$
I(t)=W^{2} A(t) s(t)^{\lambda-2} M_{2}(t)-W B(t) \frac{\dot{M}_{2}(t)}{M_{2}(t)}-W C(t) \frac{\dot{s(t)}}{s(t)} .
$$

Here the $A(t), B(t)$ and $C(t)$ are defined by

$$
\begin{aligned}
A(t)= & \frac{1}{2 M_{2}^{2}} \int_{0}^{\infty} d m_{1} d m_{2} m_{1}^{2} m_{2}^{2} K\left(\frac{m_{1}}{s(t)}, \frac{m_{2}}{s(t)}\right)\left[\left(\frac{m_{1}+m_{2}}{s(t)}\right)^{2} f\left(\frac{m_{1}+m_{2}}{s(t)}\right)-\right. \\
& \left.-\left(\frac{m_{1}}{s(t)}\right)^{2} f\left(\frac{m_{1}}{s(t)}\right)-\left(\frac{m_{2}}{s(t)}\right)^{2} f\left(\frac{m_{2}}{s(t)}\right)\right] \frac{s(t)^{4}}{m_{1}^{2} m_{2}^{2}}
\end{aligned}
$$




$$
\begin{aligned}
& B(t)=\int_{0}^{\infty} m^{2} c(m, t) f\left(\frac{m}{s(t)}\right) \\
& C(t)=\int_{0}^{\infty} m^{2} c(m, t) f^{\prime}\left(\frac{m}{s(t)}\right) \frac{m}{s(t)} .
\end{aligned}
$$

Because of the scaling hypothesis, the quantities $A(t), B(t)$ and $C(t)$ all approach limits which can be expressed as follows in terms of the scaling function $\Phi(x)$ :

$$
\begin{aligned}
& A=\int_{0}^{\infty} d x d y x K(x, y) \Phi(x) \Phi(y)[(x+y) f(x+y)-x f(x)] \\
& B=\int_{0}^{\infty} d x x^{2} \Phi(x) f(x) \\
& C=\int_{0}^{\infty} d x x^{3} \Phi(x) f^{\prime}(x) .
\end{aligned}
$$

In order to go further, we must distinguish the gelling case and the nongelling case with $\lambda=1$. Let us first consider the former. It is then reasonable to postulate that the various quantities involved such as $M_{2}(t)$ diverge as a power-law as the critical time $t_{c}$ is reached. One thus finds that

$$
\frac{\dot{M}_{2}(t)}{M_{2}(t)}=\frac{C_{1}}{t_{c}-t} \quad \frac{\dot{s}(t)}{s(t)}=\frac{C_{2}}{t_{c}-t}
$$

Assuming that the remaining term of (F.3) (proportional to $A$ ) also has the same order of magnitude yields

$$
s(t)^{\lambda-2} M_{2}(t)=\frac{C_{3}}{t_{c}-t}
$$

We now need to evaluate the different constants. An appropriate choice of $W$ allows to eliminate everything, save the ratio $C_{1} / C_{2}$, which enters via the relation

$$
M_{2}(t)=\text { const. } \cdot s(t)^{C_{1} / C_{2}}
$$

We therefore need to evaluate this exponent. To this end we make the following 
(not really rigorous) observation: from the scaling hypothesis follows

$$
\int_{\epsilon s(t)}^{\infty} m c(m, t) d m=\frac{M_{2}(t)}{s(t)} \int_{\epsilon}^{\infty} x \Phi(x) .
$$

The r.h.s of (F.9) varies as $\epsilon^{2-\tau} M_{2}(t) / s(t)$. If, however, we allow $\epsilon$ to go as $1 / s(t)$, then the l.h.s should be constant. From this we deduce

$$
\frac{C_{1}}{C_{2}}=3-\tau \text {. }
$$

For large times, (F.1) reduces to

$$
\left.\lim _{t_{1}, t_{2} \rightarrow \infty} \int_{t_{1}}^{t_{2}} \frac{d t^{\prime}}{t^{\prime}}(A-(3-\tau) B-C)\right)=0
$$

from which follows

$$
A-(3-\tau) B-C=0
$$

which is exactly (3.67) as stated in the text. Further, from (F.7), (F.8) and (F.10) follows straightforwardly

$$
\sigma=1+\lambda-\tau
$$

Now let us go over to the case in which we have $\lambda=1$. In this case we know that $\tau$ is in a sense 2, but this is not appropriate for convergence in first moment, since in that case we must always have

$$
\int_{0}^{\infty} x \Phi(x) d x<\infty
$$

So let us consider instead convergence in second moment. Up to (F.5) everything remains strictly as above. Now we must attempt to compare the different contributions. Let us first find a connection between $M_{2}(t)$ and $s(t)$. Following the same line of reasoning as above, we obtain from (F.9), which is also valid in this case, that

$$
M_{2}(t)=\text { const. } \cdot \frac{s(t)}{\ln s(t)}
$$


From this we may now deduce, by identifying the orders of magnitude of the coefficients multiplying $A$ and $C$, that

$$
\dot{s}(t)=\text { const. } \cdot \frac{s(t)}{\ln s(t)},
$$

from which finally follows

$$
s(t)=\text { const. } \cdot \exp (\text { const. } \cdot \sqrt{t})
$$

which is the result obtained by van Dongen [108] in a slightly different fashion. We may now choose $W$ in such a way as to eliminate the various constants involved, so as finally to obtain

$$
A-B-C=0
$$

For this function we only require that $x^{2} \Phi(x)$ be integrable at the origin, so that a $\tau=2$ power-law poses no problems. If we wish to cast the definition of convergence to scaling in a form that is as similar as possible to the usual one of the non-gelling case, we may reduce it to

$$
\lim _{t \rightarrow \infty}\left[\sqrt{t} \int_{0}^{\infty} m c(m, t) f\left(m e^{-\sqrt{t}}\right)\right] d m=\int_{0}^{\infty} x \Phi(x) f(x) d x
$$

This again is identical to the ansatz discovered by van Dongen in [108]. Note, however, that the above reasoning could require modifications in the presence

of additional logarithmic corrections to the singular behaviour of $\Phi(x)$ near the origin. Whether this may haoppen or not is not clear to me at present.

\section{G The Sum Kernel: The Technique of Characteristics}

In this appendix we present the general solution of (4.34) with initial conditions given by (4.35) by the method of characteristics: if we define $\bar{\zeta}(t)$ and $\bar{F}(t)$ through the equations

$$
\begin{aligned}
& \dot{\bar{F}}=-\bar{F} \\
& \dot{\bar{\zeta}}=S_{0} e^{-t}-\bar{F},
\end{aligned}
$$


then it immediately follows that, if $F(\zeta, t)$ is any solution of $(4.34)$, then $F[\bar{\zeta}(t), t]$ satisfies (G.1). It therefore suffices to match the initial conditions. This is done as follows; define

$$
\begin{aligned}
\bar{\zeta}(0) & =\zeta_{0} \\
\bar{F}(0) & =f\left(\zeta_{0}\right)
\end{aligned}
$$

where $f(\zeta)$ is defined via (4.35). The characteristic equations (G.1), (G.2) can now straightforwardly be solved together with the initial conditions (G.3), (G.4) to yield the result given in (4.36), (4.37). Nevertheless, we present the details of a particularly straightforward way to perform this algebra, since this will be used extensively in the sequel for more complex cases.

We first display an explicit solution of (G.1,G.2) in the scaling limit. This limit will turn out to be that in which $t$ goes to infinity while the following variables

$$
-s_{0}=\zeta_{0} e^{t} \quad-s=\bar{\zeta} e^{2 t}
$$

remain constant. The solution of $(\mathrm{G} .1, \mathrm{G} .2)$ is given by

$$
\begin{aligned}
& \bar{F}=f\left(\zeta_{0}\right) e^{-t}=-s_{0} e^{-2 t} \\
& \bar{\zeta}=\left[f\left(\zeta_{0}-f(0)\right] e^{-t}+\zeta_{0}-f\left(\zeta_{0}\right)+f(0)=-e^{-2 t}\left[s_{0}+\frac{1}{2} f^{\prime \prime}(0) s_{0}^{2}\right]\right.
\end{aligned}
$$

where the first equality represents the exact solution and the second involves the scaling limit defined in (G.5). Now let us note the following fundamental relation: in the scaling limit one has, because of the defining relation for $\Phi(x)$ (3.14):

$$
\int_{0}^{\infty} d m m c(m, t) e^{m \zeta} \rightarrow \int_{0}^{\infty} x \Phi(x) e^{-\zeta x} d x .
$$

But the following relation holds for the 1.h.s. of (G.8):

$$
\int_{0}^{\infty} d m m c(m, t) e^{m \zeta}=\frac{\bar{F}_{\zeta_{0}}}{\bar{\zeta}_{\zeta_{0}}}=\frac{\bar{F}_{s_{0}}}{\bar{\zeta}_{s_{0}}}
$$

where the partial derivatives in the final expressions refer to the scaling expressions derived in (G.5). This now readily yields

$$
\mathcal{L}[x \Phi(x)](s)=\frac{1}{1+f^{\prime \prime}(0) s_{0}} .
$$


From (G.5,G.7) one now obtains the necessary relation between $s_{0}$ and $s$, namely

$$
s=s_{0}+\frac{1}{2} f^{\prime \prime}(0) s_{0}^{2},
$$

from which the final result eventually follows, namely

$$
\mathcal{L}[x \Phi(x)](s)=\frac{1}{\sqrt{1+2 f^{\prime \prime}(0) s}} .
$$

Finally, the results stated in the text can be obtained in an elementary way from this equation and (G.11).

\section{H The Parity Dependent Kernel: Scaling Theory}

In the following, we develop the technical details of the parity-dependent kernel. The kinetic equations for the concentrations read

$$
\begin{gathered}
\dot{c}_{2 j+1}=M \sum_{k=0}^{j} c_{2 k+1} c_{2(j-k)}-K c_{2 j+1} \sum_{k=0}^{\infty} c_{2 k+1}-M c_{2 j+1} \sum_{k=1}^{\infty} c_{2 k} \\
\dot{c}_{2 j}=\frac{K}{2} \sum_{k=0}^{j-1} c_{2 k+1} c_{2(j-k)-1}+\frac{L}{2} \sum_{k=1}^{j-1} c_{2 k} c_{2(j-k)}- \\
-M c_{2 j} \sum_{k=0}^{\infty} c_{2 k+1}-L c_{2 j} \sum_{k=1}^{\infty} c_{2 k} .
\end{gathered}
$$

If one introduces the generating functions

$$
\begin{aligned}
& D(\zeta, t)=\sum_{j=0}^{\infty} c_{2 j+1}(t) \zeta^{2 j+1} \\
& P(\zeta, t)=\sum_{j=1}^{\infty} c_{2 j}(t) \zeta^{2 j}
\end{aligned}
$$

and the corresponding moments

$$
\begin{aligned}
& d(t)=D(1, t) \\
& p(t)=P(1, t),
\end{aligned}
$$

one eventually finds the following equations: 


$$
\begin{aligned}
\dot{D} & =M D P-[(M p(t)+K d(t)] D \\
\dot{P} & =\frac{K}{2} D^{2}+\frac{L}{2} P^{2}-P[L p(t)+M d(t)] .
\end{aligned}
$$

Note that these are ODE, the only dependence in $\zeta$ coming in through the initial conditions. Equations (H.4) then yield the closed relations for $d(t)$ and $p(t)$ :

$$
\begin{aligned}
& \dot{d}=-K d^{2} \\
& \dot{p}=\frac{K}{2} d^{2}-\frac{L}{2} p^{2}-M p d .
\end{aligned}
$$

These can be solved exactly. For our purposes, we shall only need the following elementary facts:

$$
\begin{aligned}
d(t) & =\frac{\alpha}{1+\alpha K t} \\
\lim _{t \rightarrow \infty} p(t) / d(t) & =\tilde{p}_{\infty}=\frac{K-M}{L}\left[1+\sqrt{1+\frac{K L}{(K-M)^{2}}}\right]
\end{aligned}
$$

Here $\alpha$ is the initial value of $d(t)$, which plays no significant role in the following, since we shall always be interested in the large-time limit.

Let us now introduce

$$
\begin{aligned}
\tilde{D}(\zeta, \theta) & =D(\zeta, t) / d(t) \\
\tilde{P}(\zeta, \theta) & =P(\zeta, t) / d(t) \\
\tilde{p} & =p(t) / d(t) \\
\dot{\theta}(t) & =K d(t)
\end{aligned}
$$

One then finds:

$$
\begin{aligned}
& K \frac{d \tilde{D}}{d \theta}=M \tilde{D}(\tilde{P}-\tilde{p}) \\
& K \frac{d \tilde{P}}{d \theta}=\frac{K}{2} \tilde{D}^{2}+\frac{L}{2} \tilde{P}^{2}+(K-M-L \tilde{p}) \tilde{P}
\end{aligned}
$$

The initial conditions are very close to the solution given by $\tilde{D}=1$ and $\tilde{P}(\theta)=\tilde{p}(\theta)$. They therefore follow it for considerable time and eventually can be described as solutions of the following equations

$$
K \frac{d \tilde{D}}{d \theta}=M \tilde{D}\left(\tilde{P}-\tilde{p}_{\infty}\right)
$$




$$
K \frac{d \tilde{P}}{d \theta}=\frac{K}{2} \tilde{D}^{2}+\frac{L}{2} \tilde{P}^{2}+\left(K-M-L \tilde{p}_{\infty}\right) \tilde{P},
$$

which is a two-dimensional autonomous system. The functions $\tilde{D}$ and $\tilde{P}$ then start near the equilibrium $\tilde{D}=1$ and $\tilde{P}=\tilde{p}_{\infty}$. It is then fairly straightforward to convince oneself that letting $\zeta$ tend to one and simultaneously letting $t$ tend to infinity in such a way that $t(1-\zeta)$ remains constant will give a non-trivial limiting behaviour for both $\tilde{D}$ and $\tilde{P}$. This then is easily seen to be equivalent to the existence of a scaling limit. The evaluation of the scaling function by these means, however, seems to be a hopeless task

What is possible, however, is the evaluation of the $\tau$ and $\vartheta$ exponents for the odd and even concentrations. To this end, we assume that

$$
\begin{aligned}
\lim _{t \rightarrow \infty} \sum_{j=0}^{\infty}(2 j+1) c_{2 j+1}(t) f\left(\frac{2 j+1}{K t}\right) & =\int_{0}^{\infty} x \Phi_{d}(x) f(x), \\
\lim _{t \rightarrow \infty} \sum_{j=1}^{\infty} 2 j c_{2 j}(t) f\left(\frac{2 j}{K t}\right) & =\int_{0}^{\infty} x \Phi_{p}(x) f(x) .
\end{aligned}
$$

We may then proceed once more exactly in Appendix A in order to obtain an equation of the type of (3.29) or (3.34). The final result is

$$
\begin{aligned}
& \int_{0}^{\infty} d x x^{2} \Phi_{d}(x) f^{\prime}(x)=\frac{M}{K} \int_{0}^{\infty} d x d y x \Phi_{d}(x) \Phi_{p}(y) f(x+y)- \\
& -\int_{0}^{\infty} d x d y x \Phi_{d}(x)\left[\Phi_{d}(y)+\frac{M}{K} \Phi_{p}(y)\right] f(x) \\
& \int_{0}^{\infty} d x x^{2} \Phi_{p}(x) f^{\prime}(x)=\frac{1}{2} \int_{0}^{\infty} d x d y x\left[\Phi_{d}(x) \Phi_{d}(y)+\frac{L}{K} \Phi_{p}(x) \Phi_{p}(y)\right] f(x+y)- \\
& -\int_{0}^{\infty} d x d y x \Phi_{p}(x)\left[\frac{M}{K} \Phi_{d}(y)+\frac{L}{K} \Phi_{p}(y)\right] f(x)
\end{aligned}
$$

for arbitrary continuous functions $f(x)$. In order to fix the normalizations properly, the simplest way is to note that, from (H.11) using $f(x)=1 / x$ one finds

$$
\begin{aligned}
& \int_{0}^{\infty} \Phi_{d}(x)=\lim _{t \rightarrow \infty}[K t d(t)]=1 \\
& \int_{0}^{\infty} \Phi_{p}(x)=\lim _{t \rightarrow \infty}[K t p(t)]=\tilde{p}_{\infty} .
\end{aligned}
$$


Now, putting $f(x)$ equal to $e^{-\rho x}$ into (H.12,H.13) and using the standard approach, we find from (H.12), that all terms except the first on the r.h.s. are of the same order for large $\rho$, namely of order $\rho^{2-\tau_{d}}$. The first is manifestly always subdominant. In order to evaluate $\tau_{d}$ one then works out all the prefactors and finds in the end

$$
\tau_{d}=1-\frac{M}{K} \tilde{p}_{\infty}
$$

The equation (H.13) is a bit more complex, since one must distinguish between the case in which the first term dominates or is subdominant. All other terms are of order $\rho^{2-\tau_{p}}$, whereas the first is of order $\rho^{3-2 \tau_{d}}$. Assuming first that the first term is again subdominant leads once more, using a comparison of coefficients to

$$
\tau_{p}=2-\frac{M}{K}+\frac{L}{K} \tilde{p}_{\infty}
$$

On the other hand, if the first term dominates, a simple power-law comparison leads to

$$
\tau_{p}=2 \tau_{d}-1
$$

Finally, in order to evaluate the exponent $\vartheta$ for large cluster sizes we proceed again in the standard manner: we develop (H.12,H.13) around a singularity at some $\rho_{c}<0$. This time, it is straightforward to check that the $\vartheta_{p}$ exponent is zero in all cases. As for the $\vartheta_{d}$ exponent, one can not easily say anything, except that it must be positive. If this is so, however, then the $\vartheta$ exponent of the full distribution, given by (4.46) is equal to zero.

As a final remark concerning the scaling theory, note that equations similar to $($ H.12,H.13) can easily be derived in the much more general case

$$
K(k, l)= \begin{cases}a_{1} K_{0}(k, l) & \text { for } k \text { and } l \text { odd } \\ a_{2} K_{0}(k, l) & \text { for } k \text { and } l \text { even } \\ a_{3} K_{0}(k, l) & \text { otherwise }\end{cases}
$$

where $K_{0}(k, l)$ is a homogeneous kernel that is otherwise arbitrary. From a study of these equations, it might perhaps be shown in general under what circumstances such changes in the scaling function are to be expected. In particular, it might confirm a suspicion that this is characteristic of Type II kernels, the scaling theory of which is in any case non-universal. 
Finally, let us evaluate the non-scaling asymptotic behaviour. The easiest approach is to evaluate first the long-time behaviour of $c_{1}(t)$ and $c_{2}(t)$ and then to convince oneself through induction that this is indeed the large-time behaviour at fixed $j$ for $j$ arbitrary. From (H.1) one finds for monomers and dimers

$$
\begin{aligned}
& \dot{c}_{1}=-[K d(t)+M p(t)] c_{1} \\
& \dot{c}_{2}=\frac{L}{2} c_{1}^{2}-[M d(t)+L p(t)] c_{2}
\end{aligned}
$$

From these and (H.7) the relations (4.49) stated in the text are readily verified.

\section{The $q$-sum Solution: A Sketch}

Here we shortly sketch the derivation of the solution $(4.62,4.63)$ for the $q$-sum kernel. From (4.58) the following equation for $H(\zeta, \theta)$ follows, see (4.61):

$$
\frac{\partial H}{\partial \theta}(\zeta, \theta)=H(\zeta, \theta)[H(\zeta, \theta)-H(\zeta-1, \theta)]
$$

This equation is considerably simplified by rewriting $H(\zeta, \theta)$ in the following quotient form:

$$
H(\zeta, \theta)=\frac{h(\zeta-1, \theta)}{h(\zeta, \theta)}
$$

One then finds for $h(\zeta, \theta)$ the linear equation

$$
\frac{\partial h}{\partial \theta}(\zeta, \theta)=-h(\zeta-1, \theta)
$$

This equation is linear and translation invariant with respect to $\theta$, so that it can be solved by Fourier transform. After some work one verifies that

$$
h(\zeta, \theta)=e^{i \pi \zeta} \sum_{m=0}^{\infty} \frac{\theta^{m}}{m !} \prod_{l=m}^{\infty}\left(1-e^{b(\zeta-l)}\right)^{-1}
$$

From this it is straightforward, though somewhat tedious, to obtain the final result stated in the text. 


\section{J Crossover in the $q$-sum kernel}

In the following I wish to show that $j e^{-2 t}$ and $b j$ are the correct scaling variables for the crossover transition for the modified $q$-sum kernel $\left(2-q^{k}-\right.$ $\left.q^{l}\right) / b$. I do not claim any expression, even formal, for the scaling function: the methods I shall employ will not be precise enough to fix any kind of functional dependence. They will, however, determine the order of magnitude of various quantities, and this will be sufficient.

If the above is correct, then we expect from the definition (3.90) for the scaling in crossover, that

$$
\lim _{t \rightarrow \infty}\left\{y\left[\sum_{k=1}^{\infty} c_{k}(t)\right] H\left(\zeta, \theta ; y e^{-2 t}\right)\right\}=\int_{0}^{\infty} \Phi(x, y)\left(e^{\zeta x y}-1\right) d x
$$

where we have singled out the dependence of $H(\zeta, \theta ; b)$ on the argument $b$. To show this, I shall prove the following: first I shall identify the crossover regime in time as given by finite values of the parameter

$$
\xi=\frac{b \theta-1}{\sqrt{b}}
$$

In other words, we require $b \theta$ to be close to one and the difference of order $b^{1 / 2}$. Then I show that in this case one has

$$
\left[\sum_{k=1}^{\infty} c_{k}(t)\right]^{-1}=O\left(b^{-1 / 2}\right)
$$

and finally I show that in the crossover regime as defined above one has for the generating function $H$ :

$$
H(\zeta, \theta)=\sqrt{b} \phi(\zeta, \xi)
$$

Here and throughout this appendix ${ }^{23} \phi$ will denote an arbitrary function of the scaling variables $\zeta$ and $\xi$, which may vary from equation to equation.

Let us first start with the elementary identity:

$$
\frac{1}{e_{q}\left(q^{\alpha}\right)} \sum_{r=0}^{\infty} \frac{q^{r \alpha} f\left(q^{r}\right)}{(q ; q)_{r}}=\sum_{m=0}^{\infty} \frac{f^{(m)}(0)}{m !}\left(q ; q^{\alpha}\right)_{m}
$$

$\overline{23}$ but nowhere else in this paper! 
valid for any entire function $f$ for which one of the sides converges. This is obtained by developing $f$ in a Taylor series, inverting the two sums and using the two definitions of the $q$-exponential given in (4.60).

Using (J.5) one obtains from 4.64) the following expression for $t$

$$
t=\sum_{m=0}^{\infty} \frac{(b \theta)^{m+1}}{m+1} \prod_{l=1}^{m}\left(\frac{1-q^{l}}{b l}\right)
$$

Note the extra factor of $b$ due to the fact that we are working with a modified kernel with an additional factor $b^{-1}$ in its definition. It is now easy to convince oneself that the quantity

$$
\prod_{l=1}^{m}\left(\frac{1-q^{l}}{b l}\right)
$$

decays rapidly when $m$ becomes significantly larger than $\sqrt{b}$ and is constant before. We may therefore replace, as far as orders of magnitude go, this prefactor by an appropriate cutoff of the sum, leading to

$$
t=\sum_{m=0}^{N(b)} \frac{(b \theta)^{m+1}}{m+1}
$$

where $N(b)=b^{-1 / 2}$. Using identity (P.11) one then finds

$$
t=\ln N(b)+\phi(\xi),
$$

from which (J.2) follows. An entirely similar evaluation yields (J.3) from the expression

$$
\left[\sum_{k=1}^{\infty} c_{k}(t)\right]^{-1}=\frac{1}{e_{q}(q)} \sum_{r=1}^{\infty} \frac{q^{r} e^{\theta q^{r}}}{(q ; q)_{r}} .
$$

Again, a similar transformation applied to $S(\zeta, \theta)$ yields

$$
S(\zeta, \theta)=\sum_{m=1}^{\infty}(b \theta)^{m} \frac{\left(q ; q^{-\zeta}\right)_{m}}{(q ; q)_{m}} \prod_{l=1}^{m}\left(\frac{1-q^{l}}{b l}\right) .
$$

Once more, we are led to cut the sum off at $N(b)$ leading to

$$
S(\zeta, \theta)=\sum_{m=1}^{N(b)}(b \theta)^{m} \frac{\left(q ; q^{-\zeta}\right)_{m}}{(q ; q)_{m}} .
$$


But, for this range of values of $m$ one has, as $q \rightarrow 1$,

$$
\frac{\left(q ; q^{-\zeta}\right)_{m}}{(q ; q)_{m}} \rightarrow m^{-\zeta}
$$

If one inserts (J.13) into (J.12) and reduces the sum to an integral, it follows that

$$
S(\zeta, \theta)=N(b)^{1-\zeta} \phi(\zeta, \xi)
$$

from which (J.4) follows straightforwardly. All the results initially claimed are hence established, and the statements made in the text follow.

\section{K The Simple Product Kernel: Technicalities}

In the following, we work out the detailed properties of the simple product kernel, which has all the necessary ingredients to understand the phenomenon

of gelation. It is worthwhile to do this, as the full bilinear kernel is very much more complex.

We use the generating function $G(\zeta, t)$ defined in (4.70), which satisfies the PDE (4.71). The characteristic equations of this last are given by

$$
\begin{aligned}
& \frac{d \bar{\zeta}}{d t}=-\left[\bar{G}-M_{1}(t)\right]=-(\bar{G}-1)-h(t) \\
& \frac{d \bar{G}}{d t}=0
\end{aligned}
$$

where the second equality of the first equation defines $h(t)$. From this follows immediately that

$$
G\left[\zeta_{0}-\left[g\left(\zeta_{0}\right)-1\right] t-\int_{0}^{t} h\left(t^{\prime}\right) d t^{\prime}, t\right]=g\left(\zeta_{0}\right)
$$

Setting $h(t)$ identically zero for the time being, one obtains by the inverse Laplace transform of (K.2)

$$
m c(m, t)=\frac{1}{2 \pi i} \oint_{-i \infty}^{i \infty} d \zeta_{0}\left[1-t g^{\prime}\left(\zeta_{0}\right)\right] g\left(\zeta_{0}\right) \exp \left\{-m\left[\zeta_{0}-\left(g\left(\zeta_{0}\right)-1\right) t\right]\right\}
$$


The case in which $g\left(\zeta_{0}\right)$ is $e^{\zeta_{0}}$, which corresponds to monodisperse initial conditions, is now reduced to an exercise, which the reader can do in order to verify the result (4.74) stated in the text.

We now need to gain some understanding of the failure of the solution (K.3) and of the assumption that $M_{1}(t)$ remains constant. Equations (K.1) specify the surface $G(\zeta, t)$ by displaying a pencil of straight lines as the level curves of this surface. These straight lines intersect, however, meaning that the surface must be multivalued. It is important to understand this issue well: indeed, since we have derived the PDE (4.71) without giving much thought to questionns of convergence, we must now take care that the solution we obtain has the necessary analyticity properties. We may therefore not take into consideration the second sheet of the solution, but only that sheet which connects smoothly to $\zeta=-\infty$, since there the convergence of the series is unproblematic.

We therefore need to determine the locus of points on the $(\zeta, t)$ plane for which the function $G(\zeta, t)$ becomes singular. This will define a curve, the position of which we must determine in such a way as to obtain a meaningful solution for the equations (2.2) with the kernel (4.69). The determination of the singular curve proceeds as follows: If one takes two infinitesimally close straight lines from the pencil defined by the first equation (K.1), their intersection belongs to the singular curve, as is intuitively clear and readily verified analytically ${ }^{24}$. Take $\zeta_{0}$ and $\zeta_{1}$ two infinitely close initial values for the first of the equations (K.1). This yields for the time at which these intersect

$$
t_{c}\left(\zeta_{0}\right)=1 / g^{\prime}\left(\zeta_{0}\right)
$$

and hence

$$
\zeta_{c}\left(\zeta_{0}\right)=\zeta_{0}-\frac{g\left(\zeta_{0}\right)-1}{g^{\prime}\left(\zeta_{0}\right)}-\int_{0}^{1 / g^{\prime}\left(\zeta_{0}\right)} h\left(t^{\prime}\right) d t^{\prime} .
$$

As long as $t<t_{c}(0)$, therefore, there is no problem and the solution (K.3) is valid. This is due to the fact that the function $G(\zeta, t)$ is analytic at $\zeta=0$ for such times, so that the PDE (4.71) can be applied in order to show that $G(0, t)$, which is the first moment, is indeed constant. This therefore singles

\footnotetext{
${ }^{24}$ Note that a similar pencil exists in the sum kernel and also has intersections at finite time. The reason this creates no problem is that, for the sum kernel, all the computations are carried out using a rescaled time $\theta$, and all such intersections occur at values of $\theta$ which do not correspond to any physical time. See Appendix G for the detailed definitions.
} 
out $t_{c}(0)$, which is given by

$$
t_{c} \equiv t_{c}(0)=1 / g^{\prime}(0)=M_{2}(0)^{-1}
$$

as the limiting time beyond which (K.3) ceases to hold. It is known as the gel time, and its determination is in general not a trivial task; (K.6) solves it for arbitrary initial conditions in the case of the product kernel (4.69).

Beyond $t_{c}$, we require a condition that allows to determine the amount of mass carried to infinity by the run-away aggregation process. The following remark allows to do this: since $G(\zeta, t)$ is a Laplace transform of a positive real function, its singularity nearest to the origin must be on the real $\zeta$ axis. We may therefore identify the singular curve $\left(\zeta_{c}\left(\zeta_{0}\right), t_{c}\left(\zeta_{0}\right)\right)$, as defined in $(\mathrm{K} .5, \mathrm{~K} .6)$, as giving the nearest singularity in $\zeta$ of $G(\zeta, t)$ for fixed $t$. Here $\zeta_{0}$ is simply a parameter for this curve. But since $c(m, t)$ can at most decay as a power-law, since otherwise $M_{1}(t)$ would remain constant, and since on the other hand $c(m, t)$ cannot grow exponentially, we are left with the necessary condition that the singular curve coincide with the $\zeta=0$ line on the $(\zeta, t)$ plane for $t \geq t_{c}$. In other words

$$
\zeta_{c}\left[\zeta_{0}(t)\right]=0,
$$

where one defines $\zeta_{0}(t)$ through the relation

$$
t=1 / g^{\prime}\left(\zeta_{0}(t)\right)
$$

$h(t)$ is then given by

$$
h(t)=\frac{d \zeta_{0}(t)}{d t}-\frac{d}{d t}\left[t\left(g\left[\zeta_{0}(t)\right]-1\right)\right] .
$$

In the particular case of monodisperse initial conditions, this yields

$$
h(t)=1-t^{-1}
$$

which corresponds to the results that follows from the solution (4.74) stated in the text. To derive the solution itself once $h(t)$ is known, it is enough to proceed with the expression (K.2) in the same way as we obtained (K.3) from (K.2) under the assumption that $h(t)$ was identically zero. The final result is given by

$$
m c(m, t)=\frac{1}{2 \pi i} \oint_{-i \infty}^{i \infty} d \zeta_{0}\left[1-t g^{\prime}\left(\zeta_{0}\right)\right] g\left(\zeta_{0}\right) \times
$$




$$
\times \exp \left\{-m\left[\zeta_{0}-\left(g\left(\zeta_{0}\right)-1\right) t-\int_{0}^{t} h\left(t^{\prime}\right) d t^{\prime}\right]\right\}
$$

Again, it is straightforward to verify that the Stockmayer solution given in the text follows from (K.11) in the case of monodisperse initial conditions.

Let us now finish the subject by deriving the scaling theory for the product kernel. We shall here derive it in some detail for this simpler case and later, in Appendix M, show tersely that the general case brings nothing new. The reader who has well absorbed the following will fill in the necessary details.

Since we are dealing with a gelling system, we need to use the definition (3.15) of scaling of the $n$-th moment, for $n>1$. In the following, we will take $n$ equal to two. In principle, one could look at two limits: the large time limit, and the behaviour near $t_{c}$. In the following, we will only consider the behaviour near $t_{c}$. In fact, it turns out that the large-time behaviour is considerably simpler.

Using the monodisperse solution, the moment equations or otherwise, it is easy to see that the typical size diverges as $\left(t_{c}-t\right)^{-2}$ near $t_{c}$. We therefore wish to establish this rigorously for all initial conditions, and to obtain the scaling function. To this end we first need to compute the second moment. Using the moment equations (3.157) we find for the times before $t_{c}$

$$
M_{2}(t)=\frac{M_{2}(0)}{1-M_{2}(0) t}=\left(t_{c}-t\right)^{-1}
$$

The limiting statement predicted by the definition of scaling in second moment is therefore given by

$$
\lim _{t \rightarrow t_{c}}\left\{\left(t_{c}-t\right)^{-1}\left[G\left(s\left(t_{c}-t\right)^{2}, t\right)-1\right]\right\}=\int_{0}^{\infty} x \Phi(x)\left(e^{s x}-1\right) d x
$$

To evaluate the function $G$ at this value of its arguments we must solve

$$
\zeta_{0}-\left[g\left(\zeta_{0}\right)-1\right] t=s\left(t_{c}-t\right)^{2}
$$

Remembering that $t_{c}$ is $1 / g^{\prime}(0)$, developing everything to lowest order and introducing $w$ for $\left(t_{c}-t\right)^{-1} \zeta_{0}$, we reduce (K.14) to

$$
g^{\prime \prime}(0)-2 g^{\prime}(0)^{2} w+2 g^{\prime}(0) s=0 .
$$


This then leads to

$$
\int_{0}^{\infty} x \Phi(x)\left(e^{s x}-1\right) d x=g^{\prime}(0) w=\frac{1}{\beta}[1-\sqrt{1-2 \beta s}]
$$

where $\beta$ is defined as

$$
\beta=\frac{g^{\prime \prime}(0)}{g^{\prime}(0)^{3}}
$$

and from this finally follows the expression (4.78) given in the text.

\section{The Product Kernel: Power-law initial Conditions}

In the following we treat the effect of power-law initial conditons on the gelling transition. For simplicity, we limit ourselves to the case of the product kernel. We shall use all the notation of Appendix K. We shall start from the assumption that the large $m$ behaviour of $c(m, t)$ is given by

$$
c(m, t)=A m^{-1-\alpha}[1+o(1)],
$$

which implies for the generating funcion $g\left(\zeta_{0}\right)$

$$
g\left(\zeta_{0}\right)= \begin{cases}{\left[1+\zeta_{0} g^{\prime}(0)+A \Gamma(1-\alpha)\left(-\zeta_{0}\right)^{\alpha-1}\right][1+o(1)]} & (2<\alpha<3) \\ {\left[1-A \Gamma(1-\alpha)\left(-\zeta_{0}\right)^{\alpha-1}\right][1+o(1)]} & (1<\alpha<2)\end{cases}
$$

We shall treat the two cases separately: in the first $(2<\alpha<3)$ the third moment is infinite, so that the scaling approach developed in Appendix K fails, but the second moment is finite, so that the derivation of the gel time in (K.6) holds good. In the second case $(1<\alpha<2)$, on the other hand, the second moment diverges. From a generalization of (K.6) one is therefore tempted to think that instantaneous gelation takes place, an impression that shall indeed be confirmed in this Appendix.

In the first case, we must solve the equation

$$
\zeta_{0}-\left[g\left(\zeta_{0}\right)-1\right] t=s\left(t_{c}-t\right)^{1 / \sigma},
$$


where $\sigma$ is as yet unknown. Developing to leading order in $\zeta_{0}$ and $t_{c}-t$ results in:

$$
A t_{c} \Gamma(1-\alpha)\left(-\zeta_{0}\right)^{\alpha-1}-\frac{\left(t_{c}-t\right) \zeta_{0}}{t_{c}}+s\left(t_{c}-t\right)^{1 / \sigma}=0
$$

The three terms are of the same order if we introduce the rescaled variable $w$ as $\zeta_{0}\left(t_{c}-t\right)^{1 /(\alpha-2)}$ and take

$$
\sigma=\frac{\alpha-2}{\alpha-1}
$$

The scaled version of (L.4) then reads

$$
A t_{c} \Gamma(1-\alpha)(-w)^{\alpha-1}-\frac{w}{t_{c}}+s=0
$$

From the definition of scaling, one obtains using the same reasoning as in the derivation of (K.16)

$$
\int_{0}^{\infty} x \Phi(x)\left(e^{-s x}-1\right) d x=g^{\prime}(0) w
$$

From this and (L.6) one could presumably obtain the exact form of $\Phi(x)$. This task is left to the enthusiastic reader. On the other hand, it follows from (L.6) that $-w$ goes as $s^{1 /(\alpha-1)}$ as $s \rightarrow \infty$, so that the $\tau$ exponent can be found easily, and is given by

$$
\tau=\frac{2 \alpha-1}{\alpha-1}
$$

Note that these results are in agreement with the scaling law (3.25) connecting $\tau$ and $\sigma$ in the gelling case, which in this case is given by

$$
3-\tau=\sigma
$$

In order to evaluate the correction to scaling exponent $\Delta$, we remark that the subdominant behavior of $-w$ as a function of $s$ for $s \rightarrow \infty$ is linear in $s$, so that

$$
\Delta=\frac{\alpha-2}{\alpha-1}
$$


From (L.10) follows that $\Delta$ and $\sigma$ are identical, as predicted by the scaling theory developed in the text.

Now let us evaluate the behaviour of $h(t)$ shortly beyond the gel time. To this end we use (K.9), where we first determine the behaviour of $\zeta_{0}(t)$ in this region. Since we are near the gel time, $\zeta_{0}(t)$ is small, so that we may use the asymptotic expressions (L.2) for $g\left(\zeta_{0}\right)$. Using these yields

$$
h(t)=\text { const. } \cdot\left(t-t_{c}\right)^{1 /(\alpha-2)}
$$

This implies that the flow of mass to infinity is slower than in the case where the initial conditions are short-range, which does seem rather unexpected. In particular, this is an instance in which the flow rate of sol to gel is zero at the gel time, therefore strongly suggesting that $\tau>5 / 2$, as is indeed borne out by (L.8). On the other hand, it is of course readily verified that, past the gel time, the singularity of $G(\zeta, t)$ at $\zeta=0$ is always of square root type, so that the large $j$ behaviour of the cluster size distribution for fixed times larger than $t_{c}$ always has an asymptotic power law decay as $j^{-5 / 2}$.

Let us now turn to the second case. There it follows from (L.2) that the generalization of (K.4) is

$$
t_{c}\left(\zeta_{0}\right)=\frac{\left(-\zeta_{0}\right)^{2-\alpha}}{(\alpha-1) \Gamma(2-\alpha)}
$$

From this it now follows along the same lines as those presented above or in Appendix K, that

$$
h(t)=\text { const. } \cdot t^{1 /(2-\alpha)}
$$

for small values of $t$, and that $t_{c}$ is indeed zero. Again this implies that the flow rate of sol to gel is initially zero, in remarkable contrast to the case in which the initial conditions are short range. This underlines the arbitrariness of the assumption (often made in the literature, see in particular [58]) that the flow rate at $t_{c}$ is of order unity.

\section{The Full Bilinear Kernel: A Sketch of the Scaling Theory}

In this Appendix, we give a very short argument showing that the full bilinear kernel scales in the same way as the product kernel. As the calculations become quite involved I shall be brief. 
If we define as usual

$$
G(\zeta, t)=\int_{0}^{\infty} c(m, t)\left(e^{\zeta m}-1\right)
$$

one obtains the following PDE gor $G(\zeta, t)$

$$
G_{t}=\frac{1}{2} G^{2}-B G\left(G_{\zeta}-1\right)-\frac{1}{2} C G_{\zeta}^{2}
$$

The characteristic equations for this PDE can be written as follows:

$$
\begin{aligned}
\frac{d \bar{\zeta}}{d t} & =-\bar{G}-C(q-1) \\
\frac{d p}{d t} & =A p \bar{G}+B p(q-1) \\
\frac{d q}{d t} & =A q G+B q(q-1) \\
\frac{d G}{d t} & =p-q[B G+C(q-1)]
\end{aligned}
$$

The curves defined by the above system of ODE's has properties similar to those of the characteristic equations in the quasilinear case. Specifically, one has

$$
\begin{aligned}
G[\bar{\zeta}(t), t] & =\bar{G}(t) \\
G_{t}[\bar{\zeta}(t), t] & =p(t) \\
G_{\zeta}[\bar{\zeta}(t), t] & =q(t)
\end{aligned}
$$

In order to prove that gelation occurs at finite time, it is sufficient to look at the moment equations (3.157). In order to show that the scaling theory is the same, it is enough to show that the surface described by $q$ (which corresponds to the function $G$ of Appendix $\mathrm{K}$ has the same geometrical structure as the function $G$ described in Appendix K.

In order to see this, one makes the following elementary remarks: for the usual initial conditions with $\bar{\zeta}_{0}$ negative but very small, both $q$ and $\bar{G}$ are small and negative. It therefore follows that $d q / d t$ is also small and negative. Since the gel time is finite, this means that $q$ is still of order $\bar{\zeta}_{0}$ at the gel time. Of $\bar{G}$ we can at least say that it is no larger than of order $\bar{\zeta}_{0}$. The behaviour of $\bar{\zeta}(t)$ is therefore qualitatively similar to the one found for the product kernel in Appendix $\mathrm{K}$, that is, the integral curves for $\bar{\zeta}(t)$ will cross at some finite time, which becomes the gel time as $\zeta_{0} \rightarrow 0$. We will therefore observe a similar 
backbending of the surface described by $q$ and hence a similar square root singularity, which will therefore lead to the same scaling function.

\section{N The $B \rightarrow 0$ Crossover When $C=0$}

In this appendix we study the crossover behaviour of the kernel

$$
K\left(m, m^{\prime}\right)=1+\epsilon\left(m+m^{\prime}\right)
$$

For this we use the generating function

$$
G(\zeta, t ; \epsilon)=\int_{0}^{\infty} d m^{\prime} c\left(m^{\prime}, t\right)\left[e^{m^{\prime} \zeta}-1\right]
$$

where the $\epsilon$ dependence as been explicitly put in, as we shall have occasion to single it out in the sequel. $G(\zeta, t)$ then satisfies the following PDE

$$
G_{t}-\epsilon G_{\zeta}=\frac{G^{2}}{2}-\epsilon G
$$

The characteristic equations are given by

$$
\begin{aligned}
\frac{d \bar{\zeta}}{d t} & =-\epsilon \bar{G} \\
\frac{d \bar{G}}{d t} & =\frac{\bar{G}^{2}}{2}-\epsilon \bar{G}
\end{aligned}
$$

These equations have, as always, the property that

$$
G(\bar{\zeta}(t), t ; \epsilon)=\bar{G}(t: \epsilon)
$$

We wish to show that scaling holds with $s(t)$ equal to $t$. If this is true, it follows, using the definition (3.90) of the scaling limit in crossover, that

$$
\lim _{t \rightarrow \infty}[t G(s / t ; y / t)]=\int_{0}^{\infty} d x \Phi(x, y)\left(e^{s x}-1\right)
$$

Note that this is a legitimate use of (3.90), since it involves using as a function $f(x)$ the expression $\left(e^{x}-1\right) / x$, which is indeed bounded at the origin, as required. 
From (N.5) and (N.6) it follows that we need to evaluate $t \bar{G}(t)$ as $t \rightarrow \infty$ while $\epsilon t$ is kept equal to $y$ and $t \bar{\zeta}(t)$ is equal to $s$. This means that the initial conditions $\bar{\zeta}_{0}$ and $\bar{G}_{0}$ are varied appropriately. On the other hand, we have, as always,

$$
G\left(\bar{\zeta}_{0}, 0: \epsilon\right)=\bar{G}(0: \epsilon)=\int d m c(m, 0)\left(e^{\bar{\zeta}_{0} m}-1\right)=\bar{\zeta}_{0}+O\left(\bar{\zeta}_{0}^{2}\right)
$$

We now solve (N.4) explicitly with the initial conditions given by (N.7), where we shall neglect the higher order terms:

$$
\begin{aligned}
\bar{G}(t ; \epsilon) & =\frac{2 \epsilon \bar{\zeta}_{0} e^{-\epsilon t}}{\bar{\zeta}_{0} e^{-\epsilon t}-\left(\bar{\zeta}_{0}-2 \epsilon\right)} \\
\bar{\zeta}(t ; \epsilon) & =\bar{\zeta}_{0}+2 \epsilon \ln \left(\frac{\bar{\zeta}_{0} e^{-\epsilon t}-\left(\bar{\zeta}_{0}-2 \epsilon\right)}{2 \epsilon}\right)
\end{aligned}
$$

From these results one finds that in the scaling limit it is necessary to take $\bar{\zeta}_{0}$ of order $1 / t$ (note here in passing the considerable difference to the similar computations when the ordinary scaling limit is taken in the sum kernel: in that case one finds a quadratic relationship between $\bar{\zeta}_{0}$ and $\bar{\zeta}(t)$. Again this shows that we are in two quite different sorts of asymptotic regimes). If we now define $w$ as the value at which we keep $t \bar{\zeta}_{0}$ fixed, we obtain in the scaling limit

$$
\begin{aligned}
& s=t \bar{\zeta}(t)=w+2 y \ln \left(\frac{w e^{-y}-(w-2 y)}{2 y}\right) \\
& t \bar{G}(t ; \epsilon)=\frac{2 y w e^{-y}}{w e^{-y}-(w-2 y)}
\end{aligned}
$$

From (N.6) and (N.9) one finally obtains, by substracting from the r.h.s. of (N.6) its limit as $s \rightarrow \infty$

$$
\int_{0}^{\infty} d x \Phi(x, y) e^{s x}=\frac{4 y^{2} e^{-y}}{\left(1-e^{-y}\right)\left[2 y-w\left(1-e^{-y}\right)\right]}
$$

This together with the first of the relations (N.9) yields the result stated in the text, by using the following inverse transform

$$
\Phi(x, y)=\frac{1}{2 \pi i}\left(\frac{4 y^{2}}{1-e^{-y}}\right) \oint_{c-i \infty}^{c+i \infty} d w \frac{d s}{d w} \frac{e^{-s(w) x}}{2 y-w\left(1-e^{-y}\right)}
$$


This integral can be evaluated and yields the result (4.83) as stated in the text.

\section{O The $C \rightarrow 0$ Crossover When $B=0$}

Here we treat the crossover from constant to product kernel defined by the kernel (4.80). We start by defining the following generating function:

$$
G(\zeta, t)=\int_{0}^{\infty} d m c(m, t)\left[e^{m t}-1\right] .
$$

For this function one finds, in the pre-gel regime, which is the only one we shall be concerned with here:

$$
G_{t}=\frac{1}{2}\left[G^{2}+\epsilon^{2}\left(G_{\zeta}-1\right)^{2}\right]
$$

We may now write the characteristic equations for this system, which is now quasilinear, in contrast to the others treated thus far. One finds

$$
\begin{aligned}
\dot{p} & =p \bar{G} \\
\dot{q} & =q G \\
\dot{\bar{\zeta}} & =-\epsilon^{2} \phi \\
\dot{\bar{G}} & =p-\epsilon^{2} \phi(1+\phi) .
\end{aligned}
$$

Here $p$ and $q$ denote $G_{t}$ and $G_{\zeta}$ respectively. Solving these equations with the appropriate initial conditions yields the usual property for the characteristic curves namely that they generate a solution surface:

$$
G[\bar{\zeta}(t), t]=\bar{G}(t)
$$

We also introduce the important auxiliary variable $\phi=q-1$, which satisfies

$$
\dot{\phi}=\bar{G}(1+\phi)
$$

As a first step, we want to scale away the parameter $\epsilon$ from the equations. This can be done via the following transformations

$$
y=\epsilon t \quad \tilde{p}=\frac{p}{\epsilon^{2}} \quad \tilde{G}=\frac{\bar{G}}{\epsilon^{2}} \quad \tilde{\zeta}=\frac{\zeta}{\epsilon}
$$


After these substitutions, the rescaled quantities satisfy (O.3-O.6) with $\epsilon$ equal to one. The initial conditions are now given by

$$
\begin{aligned}
\tilde{G}_{0} & =\tilde{\zeta}_{0} \\
\phi_{0} & =\mu_{2} \tilde{\zeta}_{0} \epsilon \\
\tilde{p}_{0} & =\frac{1}{2} \tilde{\zeta}_{0}^{2},
\end{aligned}
$$

where $\mu_{2}$ denotes the second moment of the initial conditions. It readily follows from (O.3) and (O.4) that $q$ and $p$ are proportional. If one now substitutes

$$
\frac{d}{d t} \ln \tilde{p}=\tilde{G}
$$

into (O.6) one obtains using the proportionality of $p$ and $q$

$$
\frac{d^{2}}{d t^{2}} \ln p=p-\frac{2}{\tilde{\zeta}_{0}^{2}} p\left[\frac{2}{\tilde{\zeta}_{0}^{2}} p-1\right]
$$

This can be straightforwardly solved by noticing the analogy to a Newtonian equation of motion and using conservation of energy. This eventually leads to

$$
\tilde{p}=\frac{\tilde{\zeta}_{0}^{2}}{2+\tilde{\zeta}_{0}^{2}-\left(\tilde{\zeta}_{0}^{2} \cos y+2 \tilde{\zeta}_{0} \sin y\right)},
$$

from which the result stated in the text readily follows upon noticing that, from the definition of the crossover limit

$$
\int_{0}^{\infty} d x x \Phi(x, y) e^{-s x}=q(-s, y)
$$

where $q(\tilde{\zeta}, y)$ is precisely the function defined above in the scaling described in $(\mathrm{O} .9)$.

\section{P The Solution of the Finite Constant Kernel}

The equations for the finite constant kernel may be written as follows:

$$
\dot{c_{j}}(t)=\frac{1}{2} \sum_{k=1}^{j-1} c_{k} c_{j-k}-c_{j}(t) S_{N}(t) \quad(1 \leq j \leq N)
$$




$$
S_{N}(t)=\sum_{k=1}^{N} c_{k}(t)
$$

The strategy, which in fact is the one that generalizes straightforwardly to all other $K_{\alpha}^{(N)}(k, l)$ is the following: first one evaluates $c_{j}(t)$ for an arbitrary function $S_{N}(t)$ using only (P.1). One then considers (P.2) as a self-consistency equation for $S_{N}(t)$. After having solved for it, one has an explicit expression for $S_{N}(t)$ and hence for $c_{j}(t)$.

In the case of (P.1) the first step is reasonably easy: one transforms to new dependent and independent variables as follows

$$
\begin{aligned}
& \phi_{j}(\theta)=c_{j}(t) \exp \left(\int_{0}^{t} S_{N}\left(t^{\prime}\right) d t^{\prime}\right) \\
& d \theta=\frac{1}{2} \exp \left(-\int_{0}^{t} S_{N}\left(t^{\prime}\right) d t^{\prime}\right) d t .
\end{aligned}
$$

Equations (P.1) then become

$$
\frac{d \phi_{j}}{d \theta}=\sum_{k=0}^{j-1} \phi_{k} \phi_{j-k}
$$

which, for monodisperse initial conditions, have the solution

$$
\phi_{j}(\theta)=\theta^{j-1} \text {. }
$$

The self-consistency condition (P.2) can now be written as

$$
S_{N}(\theta)=2 \frac{d \theta}{d t} \sum_{k=1}^{N} \theta^{k-1}
$$

One now notes that

$$
S_{N}(\theta)=-\frac{d}{d t} \ln \frac{d \theta}{d t}=-\left(\frac{d \theta}{d t}\right)^{-1} \frac{d^{2} \theta}{d t^{2}}
$$

If we now substitute (P.7) into (P.6) one obtains, after transforming to the variables $p=d \theta / d t$ and $\theta$

$$
\frac{d p}{d \theta}=-2 p \sum_{k=1}^{N} \theta^{k-1}
$$


from which the formulae given in the text follow immediately.

We now proceed to give the asymptotic expressions for the sum

$$
\chi(\theta)=\sum_{k=1}^{N} \frac{\theta^{k}}{k}-\ln N
$$

in the scaling regime, that is, when $\xi$ defined as $N(1-\theta)$ is of order one and $N \rightarrow \infty$. We first observe that

$$
\begin{aligned}
\sum_{k=1}^{N} \frac{\theta^{k}-1}{k} & =\int_{1}^{\theta} \frac{y^{N}-1}{y-1} \\
& =\int_{0}^{-\xi}\left[\left(1+\frac{y}{N}\right)^{N}-1\right] \frac{d y}{y} \\
& =\int_{0}^{-\xi} \frac{e^{y}-1}{y} d y+O\left(N^{-1}\right),
\end{aligned}
$$

where in the last equality one uses the scaling limit. The identity stated in the text then follows straightforwardly.

\section{Q The Finite-sum Kernel}

The general equations for the finite sum kernel read as follows

$$
\dot{c}_{j}=\sum_{k=1}^{j-1} k c_{k} c_{j-k}-j c_{j} M_{0}(t)-c_{j} M_{1}(t),
$$

where, originally, $M_{0}(t)$ and $M_{1}(t)$ are arbitrary functions. We shall then impose the self-consistency conditiions

$$
\begin{aligned}
& M_{0}(t)=\sum_{j=1}^{N} c_{j}(t) \\
& M_{1}(t)=\sum_{j=1}^{N} j c_{j}(t) .
\end{aligned}
$$

The first step is accomplished as follows: introduce the new variables 


$$
\begin{aligned}
\phi_{j}(\theta) & =c_{j}(t) \exp \left\{\int_{0}^{t} d t^{\prime}\left[j M_{0}\left(t^{\prime}\right)+M_{1}\left(t^{\prime}\right)\right]\right\} \\
d \theta & =\exp \left[-\int_{0}^{t} d t^{\prime} M_{1}\left(t^{\prime}\right)\right] .
\end{aligned}
$$

One then finds, denoting the derivative with respect to $\theta$ by a prime

$$
\begin{aligned}
\phi_{j}^{\prime} & =\sum_{k=1}^{j-1} k \phi_{k} \phi_{j-k} \\
\phi_{j}(0) & =\delta_{j, 1},
\end{aligned}
$$

from which one immediately obtains the solution by induction

$$
\phi_{j}(\theta)=a_{j} \theta^{j-1}
$$

where the $a_{j}$ satisfy the relation

$$
(j-1) a_{j}=\sum_{k=1}^{j-1} k a_{k} a_{j-k}
$$

If one defines

$$
F(\zeta)=\sum_{j=1}^{\infty} a_{j} e^{j \zeta},
$$

then one finds immediately that

$$
F e^{-F}=e^{\zeta}
$$

from which both the explicit expressions for the $a_{j}$ cited in the text as well as the various other statements made there are easily derived.

Let us now define

$$
\begin{aligned}
& X=\exp \left\{\int_{0}^{t} d t^{\prime}\left[M_{1}\left(t^{\prime}\right)\right]\right\} \\
& Y=\exp \left\{\int_{0}^{t} d t^{\prime}\left[M_{0}\left(t^{\prime}\right)\right]\right\}
\end{aligned}
$$


From this we derive the equations

$$
\begin{aligned}
& X^{\prime}=X \sum_{j=1}^{N} j a_{j} \theta^{j-1} Y^{-j} \\
& Y^{\prime}=\sum_{j=1}^{N} a_{j} \theta^{j-1} Y^{1-j}
\end{aligned}
$$

If we now introduce $\Lambda=Y / \theta$, we obtain the solution for $\Lambda$ described in the text from the second equation (Q.11). From the first equation (Q.11) one obtains

$$
d \ln \theta=\frac{d \ln X}{\sum_{j=1}^{N} j a_{j} \Lambda^{-j}} .
$$

Identifying this with the correponding expression of $d \ln \theta$ in terms of $\Lambda$ one eventually obtains

$$
X=\frac{1}{1-\sum_{j=1}^{N} a_{j} \Lambda^{-j}},
$$

from which all results quoted in the text follow.

\section{R The Three-body Kernel $m_{1}+m_{2}+m_{3}$}

If we define $G$ to be $F(\zeta, t)-S(t)$, with the definitions given in the text, we find that the characteristic equations for the PDE satisfied by $G$ are as follows

$$
\begin{aligned}
\dot{\bar{\zeta}} & =-\frac{1}{2} \bar{G}(\bar{G}+2 S) \\
\dot{\bar{G}} & =-S \bar{G} \\
\dot{S} & =-S^{2} .
\end{aligned}
$$

Here $S$ is defined as in (4.105). Let us introduce $\theta$ through the equation

$$
d \theta=S(t) d t
$$

From this the general solution follows straightforwardly:

$$
\begin{aligned}
\bar{\zeta} & =\zeta_{0}-\frac{1}{2} \frac{f\left(\zeta_{0}\right)^{2}-f(0)^{2}}{f(0)}\left(1-e^{-\theta}\right) \\
\bar{G} & =\left[f\left(\zeta_{0}\right)-f(0)\right] e^{-\theta}
\end{aligned}
$$


In order to compute the exact solution for the monodisperse initial condition, one first sets $f\left(\zeta_{0}\right)$ equal to $e^{\zeta_{0}}$ in (R.4) and then uses the following representation for the concentrations $c_{j}(t)$ :

$$
c_{j}(t)=\frac{1}{2 \pi i} \oint_{C} G(\zeta, t) e^{-j \zeta} d \zeta
$$

where the contour $C$ runs from $-a-\pi i$ to $-a+\pi i$, where $a$ is an arbitrary real number, which should be large enough for all sums to converge. We then use the basic properties of characteristic equations, that is,

$$
G\left[\zeta\left(\theta, \zeta_{0}\right), \theta\right]=\bar{G}\left(\theta, \zeta_{0}\right)
$$

Here, both $\zeta\left(\theta, \zeta_{0}\right)$ and $\bar{G}\left(\theta, \zeta_{0}\right)$ are explicitly known functions of $\zeta_{0}$ via (R.4). It is therefore possible to rewrite the integral (R.5) in the $\zeta_{0}$ variables. After some fairly tedious but straightforward evaluations of contour integrals, the result (4.109) is obtained. This method is quite general and can be used to evaluate virtually all kernels for which the generating function is obtained via characteristics. It is, however, almost invariaby quite cumbersome, as this example shows.

In order to prove scaling, we proceed entirely as in the sum kernel case (see appendix $\mathrm{G}$ for the details of the approach followed, as well as the notation.) The scaling limit is defined by

$$
s=-\zeta e^{2 \theta} \quad s_{0}=-\zeta_{0} e^{\theta} .
$$

In this limit (R.3) yields the following relation

$$
s=s_{0}+\frac{f^{\prime \prime}(0)}{2} s_{0}^{2}
$$

By the usual techniques this yields the results stated in the text.

If, on the other hand, we start with initial conditions of the type

$$
c(m, 0)=A m^{-\alpha} \quad(2<\alpha<3)
$$

one finds for large $t$ and hence large $\theta$, that

$$
A \Gamma(1-\alpha)\left(-\zeta_{0}\right)^{\alpha-1}+\zeta_{0} e^{-\theta}=\zeta
$$


Hence, in order for all the terms in (R.10) to scale similarly, one must introduce the scaling variables

$$
s=-\zeta e^{\theta(\alpha-1) /(\alpha-2)} \quad s_{0}=-\zeta_{0} e^{\theta /(\alpha-2)} .
$$

From this immediately follows that

$$
z=\frac{\alpha-1}{\alpha-2} \quad \tau=\frac{\alpha}{\alpha-1}
$$

\section{S The Kernel $m_{1}+m_{2}+m_{3}$ Perturbing the Constant Kernel}

We wish to show here the scaling behaviour of the mixed two- and three-body system described in (4.117) in the specific case given by the first equation (4.117). For convenience I limit myself to the case of monodisperse initial conditions, but the general case presents no difficulties of principle. In this case, if one defines

$$
\begin{aligned}
F(\zeta, t) & =\sum_{j=1}^{\infty} c_{j}(t) e^{j \zeta} \\
S(t) & =\sum_{j=1}^{\infty} c_{j}(t) \\
G(\zeta, t) & =F(\zeta, t)-S(t),
\end{aligned}
$$

one obtains the following set of equations

$$
\begin{aligned}
G_{t}-\frac{1}{2} \alpha G(G+2 S) G_{z} & =\frac{G^{2}}{2}-\alpha S G \\
\dot{S} & =-\left(\frac{1}{2}+\alpha\right) S^{2}
\end{aligned}
$$

Applying the method of characteristics to (S.2) one gets

$$
\begin{aligned}
\dot{\bar{\zeta}} & =-\frac{1}{2} \alpha G(G+2 S) \\
\dot{\bar{G}} & =\frac{G^{2}}{2}-\alpha S G
\end{aligned}
$$

If we now introduce the rescaled time $\theta$ defined by

$$
d \theta=S(t) d t
$$


and define the initial conditions for $\bar{G}$ and $\bar{\zeta}$ to be $G_{0}$ and $\zeta_{0}$ respectively, one obtains for $\bar{G}$

$$
\begin{aligned}
\bar{G}(\bar{\zeta}, \theta) & =\frac{G_{0} e^{\theta / 2}}{1+G_{0}-G_{0} e^{\theta / 2}} e^{-(1 / 2+\alpha) \theta} \\
& \simeq \frac{\zeta_{0} e^{\theta / 2}}{1-\zeta_{0} e^{\theta / 2}} e^{-(1 / 2+\alpha) \theta}
\end{aligned}
$$

where in the second line, the smallness of $\zeta_{0}$ has been used as well as the following relation

$$
G\left(\zeta_{0}, 0\right)=e^{\zeta_{0}}-1=\zeta_{0}+\frac{1}{2} \zeta_{0}^{2}+O\left(\zeta_{0}^{3}\right)
$$

We now want to obtain scaling from this solution of (S.2). This is not quite a trivial task, so we carry it out in some detail. As was stated in (3.14), it is sufficient to show that there exists a function $s(t)$ such that

$$
\left.\lim _{t \rightarrow \infty} G_{\zeta}(\zeta)\right|_{\zeta=-s / s(t)}=\mathcal{L}[x \Phi(x)](s)
$$

We therefore wish to express the 1.h.s. of (S.9) in terms of a function of a scaling quantity alone. To do this, we first relate it to the solutions of the characteristic equations (S.4, S.5). Since, because of the definition of the characteristic equations, we have

$$
G[\bar{\zeta}(t), t]=\bar{G}(t)
$$

one verifies using L'Hospital's rule, that

$$
\lim _{\zeta_{0} \rightarrow 0} \frac{\bar{G}(t)}{\bar{\zeta}(t)}=G_{\zeta}(\bar{\zeta}(t), t)
$$

at least whenever $\bar{\zeta}(t) \rightarrow 0$ when $\zeta_{0} \rightarrow 0$ for times in the scaling limit, which is a condition we shall verify shortly.

We therefore need an expression for $\bar{\zeta}(t)$. In terms of $\theta$, the following expression follows from (S.4,S.7) after some routine algebra:

$$
\bar{\zeta}=\zeta_{0}\left[\alpha\left(-\zeta_{0}\right)^{2 \alpha} \int_{-\zeta_{0}}^{-\zeta_{0} e^{\theta / 2}} x^{-2 \alpha} \frac{2 x+3}{(1+x)^{2}} d x+e^{-\alpha \theta}\right]
$$

From now on, we restrict ourselves explicitly to the case $\alpha<1 / 2$. Then the integral in the r.h.s. of (S.12) converges at the lower end. From this further 
follows that we may take $\zeta_{0} e^{\theta / 2}$ as the scaling variable. It follows that

$$
\bar{\zeta}(\theta) e^{(1 / 2+\alpha) \theta}=Z\left(-\zeta_{0} e^{\theta / 2}\right)
$$

where $Z(x)$ is a function which is expressed in terms of quadratures as follows

$$
Z(x)=-x\left[1+\alpha x^{2 \alpha} \int_{0}^{x} d y \frac{y^{-2 \alpha}(2 y+3)}{(1+y)^{2}}\right] .
$$

Note that $\bar{\zeta}(\theta) \rightarrow 0$ as $\zeta_{0} \rightarrow 0$ at fixed value of $s_{0}=\zeta_{0} e^{\theta / 2}$. Let us now define $s$ as $\bar{\zeta}(\theta) e^{(1 / 2+\alpha) \theta}$. We then find that these two quantities are connected by

$$
s=Z\left(s_{0}\right) .
$$

We may therefore use the relation (S.11) to evaluate the r.h.s. of (S.9). This leads to

$$
\mathcal{L}[x \Phi(x)](s)=\frac{s_{0}}{Z\left(s_{0}\right)\left(1-s_{0}\right)}=\frac{s_{0}}{s\left(1-s_{0}\right)} .
$$

This gives the scaling function explicitly, although a more detailed evaluation would require considerable algebra. Since this is not our purpose here, however, we merely conclude these remarks by evaluating the large- $s$ asymptotics of $\mathcal{L}[x \Phi(x)]$, which immediately yields the value of the exponent $\tau$. But, in the limit of large $s$, one also has $s_{0} \rightarrow \infty$, so that the asymptotics of the r.h.s. of (S.16) is of order $s^{-1}$, which corresponds to the value of one for the exponent $\tau$. On the other hand, since the scaling variable is $\zeta t$, as readily follows from (S.3,S.6), it follows that the typical size grows as $t$ and hence the growth exponent is one, as stated in the text.

We now turn to the case $\alpha>1 / 2$. Since the results are less important and can be obtained by a straightforward extension of the previous approach I shall be brief. In the limit $\zeta_{0} \rightarrow 0$ the relation (S.12) reduces to

$$
\frac{3 \alpha}{1-2 \alpha} \zeta_{0}^{2}+\zeta_{0} e^{-\alpha \theta}=\zeta
$$

by extending the integral to infinity. If we therefore now define

$$
\zeta_{0}=s_{0} e^{-\alpha \theta} \quad \zeta=s e^{-2 \alpha \theta},
$$


we obtain the following relation

$$
\frac{3 \alpha}{1-2 \alpha} s_{0}^{2}+s_{0} e^{-\alpha \theta}=s
$$

If we use this in connection with (S.7), for which we did not require the hypothesis $\alpha<1 / 2$, we obtain in the scaling limit

$$
\mathcal{L}[x \Phi(x)](s)=\frac{s_{0}(s)}{s},
$$

where $s_{0}$ is computed from $s$ via (S.19). From this follows the result that $\tau=3 / 2$, as stated in the text.

\section{T The Scaling Function for the One-dimensional PCM}

Let us show how to prove that (5.19) holds in general. In this case, the first moment is equal to $\rho_{0}$, so that we should properly first divide by it to obtain the

correctly normalized scaling function. Let us compute the generaing function $G(\zeta, t)$ given by

$$
G(\zeta, t)=\rho_{0}^{-1} \sum_{k=1}^{0} k c(k, t) e^{k \zeta} .
$$

After some straightforward algebra one obtains

$$
G(\zeta, t)=\frac{4 e^{\zeta}}{\sqrt{\pi}} \int_{0}^{\infty} y^{2} \exp \left[2 \rho_{0} \sqrt{D t}\left(e^{\zeta}-1\right)\right] e^{-y^{2}} d y
$$

If one now defines

$$
s(t)=2 \rho_{0} \sqrt{D t} \quad \sigma=\zeta s(t)
$$

and noting that, as follows from the definition of scaling through generating functions stated in (3.14), one has

$$
\frac{4}{\sqrt{\pi}} \int_{0}^{\infty} y^{2} e^{-y^{2}} e^{\sigma y}=\int_{0}^{\infty} x \Phi(x) e^{\sigma x} d x
$$

from which the result stated in the text follows immediately through inverse Laplace transform. 


\section{U Ballistic Aggregation: Sketch of Exact Solution}

The analysis starts by the following basic fact: once the initial condition is fully known, the cluster size distribution function is known for all times. In the model considered in $[75,28,29,30]$, the particle positions are fixed (they occupy a lattice, which we take to have unit spacing), so that everything is reduced to averaging over the initial momenta. This is in turn considerably simplified by the assumption that the initial momenta are independent Gaussian random variables.

One must therefore express the event that, say, particles 1 to $m$ form one cluster at time $t$ in terms of the initial momenta of all particles. After this, one evaluates the probability of this event using the independence of the initial momenta. In the following, we shall make heavy use of the fact that, if a cluster has formed at time $t$, the center of mass of the particles that constitute it has been moving on a straight line with the cluster's velocity ever since the beginning.

There are three conditions (or rather, classes of conditions) which must all be satisfied for this event to occur:

(1) A set of conditions guaranteeing that the particles 1 to $m$ do in fact come to form a cluster. One sees that it is necessary and sufficient that, for all $m_{1}$, the particle cluster from 1 to $m_{1}$ have sufficient velocity to collide with the corresponding cluster from $m_{1}+1$ to $m$ before time $t$. This is easily seen to be equivalent to the following set of conditions on the velocities $v\left(m_{2}\right)$ of the particles at site $m_{2}$ with respect to the center of mass of the cluster

$$
-2 t \sum_{m_{2}=1}^{m_{1}} v\left(m_{2}\right) \leq\left(m_{1}-\frac{1}{2} m\right)^{2}-\frac{m^{2}}{4} .
$$

for all $m_{1}$. Now we see a connection with the functions defined in the text: due to the independence of the $v\left(m_{2}\right)$, the partial sums on the l.h.s. of (U.1) perform a random walk starting from the origin and returning to it. Conditions (U.1) then state that this random walk must always remain below the parabolic profile given by the r.h.s. of (U.1).

(2) A set of conditions guaranteeing that the particles to the right of the cluster will not themselves coalesce with the cluster. If we again measure all velocities with respect to that of the center of mass of the forming cluster, one obtains the following condition for all velocities of particles to the right of the cluster:

$$
-2 t \sum_{m_{2}=m+1}^{m_{1}} v\left(m_{2}\right) \leq\left[m_{1}+\left(\frac{1}{2} m+v t\right)\right]^{2}-\left(\frac{1}{2} m+v t\right)^{2}
$$


where $v$ denotes the velocity of the forming cluster in the rest frame. These conditions state that no cluster formed by particles $m+1$ to $m_{1}$ can collide before time $t$ with the center of mass of the forming cluster which, by assumption, is at rest in the frame we consider. Again, we see that a given random walk is constrained to stay below a certain parabolic profile. In this case, this must occur for all values of the upper limit of the sum, so we are dealing with events of the type described by $\mathcal{T}_{\infty}$.

(3) An entirely similar set of conditions for particles to the left of the forming cluster. The equations are, of course, entirely similar to (U.2), with $v$ replacd by $-v$.

From these considerations the results stated in the text can be obtained more or less straightforwardly. The crucial remark is the following: in the scaling limit, that is, when the cluster masses $m$ are large, and $t \gg 1$ as well, the random walks will tend, under an appropriate scaling of the variables, to a continuous diffusion. The fact that all random walks start on the absorbing parabola causes technical problems, which are excellently discussed in the various references given above.

As a final excercise for the interested reader, consider the following problem: if the initial velocities are independent, but distributed according to some Lévy stable law, what can we say about the solution? Everything up to (U.1) and (U.2) still holds. The real difficulty consists in building up a continuuum model, since there is, to my knowledge, no Lévy analogue to Brownian motion. The discrete problem, on the other hand, is quite formidable. The numerical results found in [45] make it probable that a scaling picture does indeed exist when the average velocity is still finite, whereas the other case is quite pathological.

\section{References}

[1] M. Abramowitz and I. Stegun, "Handbook of Mathematical Functions", NewYork, Dover (1972)

[2] D.J. Aldous, "Deterministic and stochastic models for coalescence (aggregation, coagulation: review of the mean-field theory for probabilists", Bernoulli 53 (1999)

[3] M. Aizenman and T.A. Bak, "Convergence to equilibrium in a system of reacting polymers", Comm. Math. Phys. 65203 (1979)

[4] T A Bak and O J Heilmann, "Post-gelation solutions to Smoluchowski's coagulation equation" J. Phys. A 274203 (1994)

[5] J.M. Ball and J. Carr, "The discrete coagulation-fragmentation equations: existence, uniqueness and density conservation", J. Stat. Phys. 61203 (1990) 
[6] E. Ben-Naim and P. L. Krapivsky, "Kinetics of aggregation-annihilation processes", Phys. Rev. E 52, 6066 (1995); (cond-mat/9507031).

[7] A.B. Boehm, C. Poor and S.B. Grant, "Particle coagulation and the memory of initial conditions", J. Phys. A 31, 9241 (1998)

[8] J.-P. Bouchaud and A. Georges, "Anomalous diffusion in disordered media: statistical mechanisms, models and physical applications", Phys. Repts 195, $127(1990)$

[9] M.L. Broide and R.J. Cohen, "Experimental Evidence of Dynamic Scaling in Colloidal Aggregation", Phys. Rev. Lett. 642026 (1990)

[10] E. Buffet and J.V. Pulé, "Gelation, the diagonal case revisited", Nonlinearity 2373 (1989)

[11] E. Buffet and J.V. Pulé, "On Lushnikov's model of gelation", J. Stat. Phys. 581041 (1990)

[12] E. Buffet and R.F. Werner, "A counterexample in coagulation theory", J. Math. Phys. 322276 (1991)

[13] F Calogero and F Leyvraz, "A new solvable model of aggregation kinetics", J. Phys. A 327697 (1999)

[14] F. Calogero and F. Leyvraz, "New Results on a Parity-dependent Model of Aggregation", J. Phys. A 335619 (2000)

[15] G.F. Carnevale, Y. Pomeau and W.R. Young, "Statistics of ballistic agglomeration", Phys. Rev. Lett. 642913 (1990)

[16] J. Carr and F.P. da Costa, "Instantaneous Gelation in Coagulation Dynamics", Z. Angew. Math. Phys 43974 (1992)

[17] Z. Cheng, S. Redner and F. Leyvraz, "Coagulation with a steady monomer point source" Phys. Rev. Lett. 62, 2321 (1989)

[18] S. Cueille and C. Sire, "Nontrivial polydispersity exponents in aggregation models", Phys. Rev. E 555465 (1997)

[19] S. Cueille and C. Sire, "Droplet nucleation and Smoluchowski's equation with growth and injection of particles", Phys. Rev. E 57881 (1998)

[20] S.C. Davies, J.R. King and J.A.D. Wattis, "The Smoluchowski coagulation equations with continuous injection", J. Phys. A 327745 (1999)

[21] C. R. Doering and D. benAvraham, "Interparticle distribution functions and rate equations for diffusion-limited reactions", Phys. Rev. A 383035 (1988)

[22] C. R. Doering and D. benAvraham, "Diffusion-limited coagulation in the presence of particle input: Exact results in one dimension", Phys. Rev. Lett. 622563 (1989)

[23] R.L. Drake in Topics in Current Aerosol Research, G.M. Hidy and J.R. Brock eds. (Pergamon Press, New York) vol. 3, part 2 (1972) 
[24] M.H. Ernst, R.M. Ziff and E.M. Hendriks, "Coagulation Processes with a Phase Transition", J. Colloid and Interface Sci. 97266 (1984)

[25] M. H. Ernst, E. M. Hendriks and F. Leyvraz, "Smoluchowski's equation and the $\theta$-exponent for branched polymers" J. Phys. A 172137 (1984)

[26] M.H. Ernst, "Kinetics of Clustering in Irreversible Aggregation", in Fractals in Physics(L. Pietronero and E. Tosatti eds. p. 289 North-Holland, Amsterdam 1986

[27] M. E. Fisher, "Walks, Walls, Wetting an Melting", J. Stat. Phys. 34667 (1984)

[28] L. Frachebourg, "Exact Solution of One-Dimensional Ballistic Aggregation", Phys. Rev. Lett. 821502 (1999)

[29] L. Frachebourg, Ph. A. Martin, and J. Piasecki, "Ballistic aggregation: a solvable model of irreversible many particles dynamics" Physica A 27969 (2000).

[30] L. Frachebourg, V. Jacquemet and Ph. A. Martin, "Inhomogeneous Ballistic Aggregation", J. Stat. Phys.105 745 (2001)

[31] S.K. Friedlander and C.S. Wang, "The self-preserving particle size distribution for coagulation by Brownian motion", J. of Colloid and Interface Sci. 22126 (1966)

[32] S.K. Friedlander, "Smoke, Dust and Haze", second edition, Oxford University Press (2000)

[33] G. Gasper and M. Rahman, Basic Hypergeometric series (Encyclopedia of Mathematics and its Applications vol. 35) ed. G.-C. Rota (Cambridge, Cambridge University Press)

[34] A. E. Hansen, D. Marteau, and P. Tabeling, "Two-dimensional turbulence and dispersion in a freely decaying system", Phys. Rev. E 587261 (1998)

[35] E.K.O. Hellén, P.E. Salmi, and M.J. Alava, "Cluster persistence in onedimensional diffusion-limited cluster-cluster aggregation", cond-mat/0206139

[36] E. Hendriks, M.H. Ernst and R.M. Ziff, "Coagulation equations with gelation", J. Stat Phys. 31519 (1983)

[37] G.R. Hidy and J.R. Brock, "The Dynamics of Aerocolloidal Systems", International Reviews in Aerosol Physics and Chemistry, Pergamon Press (1970)

[38] S. Ispolatov and P.L. Krapivsky, "Ballistic coalescence model", Physica A 252 165 (1998)

[39] A. V. Ivlev, G. E. Morfill, and U. Konopka, "Coagulation of Charged Microparticles in Neutral Gas and Charge-Induced Gel Transitions", Phys. Rev. Lett. 89, 195502 (2002) 
[40] Yu Jiang, Hu Gang, "Generalized Smoluchowski equation with gelation", Phys. Rev. B 394659 (1989)

[41] Yu Jiang and Hu Gang, "Long-time behaviour of the cluster size distribution in joint coagulation processes", Phys. Rev. B 40661 (1989)

[42] Yu Jiang, Hu Gang, and Ma Benun, "Critical behavior of the generalized Smoluchowski coagulation equation ", Commun. Theor. Phys. 12395 (1989)

[43] Yu Jiang, Hu Gang, and Ma BenKun, "Gelation in generalized Smoluchovski equation", Commun. Theor. Phys. 11255 (1989)

[44] Yu Jiang, Hu Gang and Ma BenKun, "Critical property and universality in the generalized Smoluchowski coagulation equation", Phys. Rev. B 419424 (1990)

[45] Yu Jiang and F. Leyvraz, "Scaling Theory for Ballistic Aggregation", J. Phys. A 26 L179 (1993)

[46] Yu Jiang, "Scaling theory for multipolymer coagulation", Phys. Rev. E50 4901 (1994)

[47] R. Jullier and R. Botet, "Aggregation and Fractal Aggregates", World Scientific Publishing Company (1987)

[48] K. Kang and S. Redner, "Fluctuation effects in Smoluchowski reaction kinetics", Phys. Rev. A 30302833 (1984)

[49] K. Kang, S. Redner, P. Meakin and F. Leyvraz, "Long-time crossover phenomena in coagulation kinetics", Phys. Rev. A 331171 (1986)

[50] P.L. Krapivsky, "Aggregation processes with $n$-particle elementary reactions", J. Phys. A 244697 (1991)

[51] P.L. Krapivsky and E. ben-Naim, "Aggregation with multiple conservation laws", Phys. Rev. E 53291 (1996)

[52] P.L. Krapivsky and S. Redner, "Transitional aggregation kinetics in dry and damp environments", Phys. Rev. E 543553 (1996)

[53] D.S. Krivitski, "Numerical solution of the Smoluchowski kinetic equation and asymptotics of the distribution function", J. Phys. A 282025 (1995)

[54] F.S. Lai, S.K. Friedlander, J. Pich and G.M. Hidy, "The self-preserving particle size distribution for Brownian motion in the free-molecule regime", Journal of Colloid and Interface Sci. 39, 395 (1973)

[55] M.H. Lee, "On the Validity of the Coagulation Equation and the Nature of Runaway Growth', Icarus 14374 (2000)

[56] M.H. Lee, "A survey of numerical solutions to the coagulation equation", J. Phys. A 3410219 (2001)

[57] F. Leyvraz and H.R. Tschudi, "An exactly solvable model for externally controlled coagulation”, J. Phys. A 131867 (1980) 
[58] F. Leyvraz and H.R. Tschudi, "Singularities in the kinetics of coagulation processes", J. Phys. A 143389 (1981)

[59] F. Leyvraz and H.R. Tschudi, "Critical kinetics near gelation", J. Phys. A 15 $1951(1982)$

[60] F. Leyvraz, "Existence and properties of post-gel solutions of the equations of coagulation", J. Phys. A 162861 (1983)

[61] F. Leyvraz, "Large-time behavior of the Smoluchowski equations of coagulation" Phys. Rev. A 29, 854 (1984)

[62] F. Leyvraz, "Rate equations approach to aggregation" in On Growth and Form: Fractal and Nonfractal Patterns in Nature, H.E. Stanley and N. Ostrowski eds., M. Nijhoff, Dordrecht (1986)

[63] F. Leyvraz and S. Redner, "Non-Universality and breakdown of scaling in a two-component coagulation model", Phys. Rev. Lett. 57163 (1986)

[64] F. Leyvraz and S. Redner, "Non-Universality and breakdown of scaling in two-species aggregation", Phys. Rev. A 364033 (1987)

[65] F Leyvraz, "Scaling and crossover properties of a new solvable model of aggregation kinetics", J. Phys. A 327719 (1999)

[66] T.M. Liggett, "Interacting Particle Systems" Berlin: Springer (1985)

[67] A.A. Lushnikov, "Evolution of coagulating systems", Journal of Colloid and Interface Sci. 45549 (1973)

[68] A.A. Lushnikov, "Evolution of coagulating systems 2: asymptotic size distribution and analyticity properties of generating functions", Journal of Colloid and Interface Sci. 48400 (1974)

[69] A.A. Lushnikov, "Evolution of coagulating systems 3: Coagulating Mixtures", Journal of Colloid and Interface Sci. 5494 (1976)

[70] A.A. Lushnikov, "Coagulation in finite systems", Journal of Colloid and Interface Sci. 65276 (1978)

[71] A.A. Lushnikov and J.I. Tokar, "Nonstationary conditions of coagulation in the systems with stationary external source of particles", Dokl. Akad. Nauk SSSR 252136 (1980)

[72] A.A. Lushnikov and V.N. Piskunov, "New exactly solvable models in the coagulation theory", Dokl. Akad. Nauk SSSR 267132 (1982)

[73] A. A. Lushnikov and M. Kulmala, "Singular self-preserving regimes of coagulation processes", Phys. Rev. E 65041604 (2001)

[74] A. A. Lushnikov and M. Kulmala, "Nonsingular self-preserving regimes of coagulation-condensation process", Phys. Rev. E 64031605 (2001)

[75] Ph. A. Martin and J. Piasecki, "One-dimensional Ballistic Aggregation: Rigorous Long-time Estimates", J. Stat. Phys. 76447 (1994) 
[76] J.B. McLeod, "On an infinite set of non-linear differential equations, Quart. J. Math. Oxford (2) 13119 (1962)

[77] J.B. McLeod, "On an infinite set of non-linear differential equations (II), Quart. J. Math. Oxford (2) 13192 (1962)

[78] P. Meakin, "Fractals, Scaling and Growth Far from Equilibrium", Cambridge Nonlinear Science Series 5, Cambridge University Press (1998)

[79] Z.A. Melzak, "The effects of coalescence in certain collision processes", Q. Journal of Appl. Math. 11231 (1953)

[80] M. Mobilia, P.L. Krapivsky and S. Redner, "Kinetic anomalies in additionaggregation processe", J. Phys. A (in press)

[81] J.R. Norris, 'Uniqueness, nonuniqueness and a hydrodynamic limit for the stochastic coalescent", Ann. Appl. Probab. 978 (1999)

[82] J.R. Norris, 'Cluster coagulation”, Commun. Math. Phys. 209407 (2000)

[83] P.P Oliva, D.H. Zanette and P.A. Alemany, "Analytical approach to coagulation and annihilation of particles with anomalous diffusion", Phys. Rev. E 53228 (1996)

[84] J. Piasecki, "Universal distribution of masses in a one-dimensional sticky gas" Physica (Amsterdam) 190A 90 (1992).

[85] J. Piasecki, E. Trizac and M. Droz, "Dynamics of ballistic annihilation", Phys. Rev. E 66066111 (2002)

[86] H.R. Pruppacher and J.D. Klett, "Microphysics of Clouds and Precipitation", Reidel, Dordrecht (1978)

[87] W.T. Scott, "Analytic studies on cloud droplet coalescence", J. Atmospheric Sci. 2554 (1968)

[88] M. Slemrod, "Coagulation-Diffusion Systems: Derivation and Existence of Solutions for the Diffuse-Interface Structure Equations", Physica D 46351 (1990)

[89] M. von Smoluchowski, "Drei Vorträge über Diffusion, Brown'sche Molekularbewegung und Koagulation von Kolloidteilchen", Z. Phys. 17557 (1916)

[90] J.L. Spouge, "Solutions and critical times for the monodisperse coagulation equation when $a_{i j}=A+B(i+j)+C i j$ ", J. Phys. A 16767 (1983)

[91] J.L. Spouge, "Solutions and critical times for the polydisperse coagulation equation when $a(x, y)=A+B(x+y)+C x y$ ", J. Phys. A 163127 (1983)

[92] J.L. Spouge, "Analytic solutions to Smoluchowski's coagulation equation: a combinatorial interpretation", J. Phys. A 183063 (1985)

[93] J.L. Spouge, "Exact solutions for a diffusion-reaction process in one dimension", Phys. Rev. Lett. 60871 (1988) 
[94] J.L. Spouge, "Exact solutions for diffusion-reaction processes in one dimension II: Spatial distributions", J. Phys A 214183 (1988b)

[95] W.H. Stockmayer, "Theory of Molecular Size Distribution and Gel Formation in Branched-Chain Polymers", J. Chem. Phys. 1145 (1943)

[96] D.L. Swift and S.K. Friedlander, "Coagulation of hydrosols by Brownian motion and laminar shear flow", J. Colloid and Interface Sci. 19621 (1964)

[97] D.C. Torney and H.M. McConnell, , "Diffusion-limited Reaction Rate Theory for Two-dimensional Systems", Proc. R. Soc. London, Ser. A 387147 (1983)

[98] E. Trizac and J.-P. Hansen, "Dynamic Scaling Behavior of Ballistic Coalescence", Phys. Rev. Lett. 744114 (1995).

[99] E. Trizac, private communication (2001)

[100] E. Trizac, "Kinetics and Scaling in Ballistic Annihilation", Phys. Rev. Lett. 88160601 (2002)

[101] P.G.J. van Dongen and M.H. Ernst, "Pre- and post gel size distributions in (ir)reversible polymerisation", J. Phys A 16 L327 (1983)

[102] P.G.J. van Dongen and M.H. Ernst, "Dynamic scaling in the kinetics of clustering", Phys. Rev. Lett. 541396 (1985)

[103] P.G.J. van Dongen, "Cluster size distribution in irreversible aggregation at large times", J. Phys. A 182779 (1985)

[104] P.G.J. van Dongen and M.H. Ernst, "Fluctuations in coagulating systems", J. Stat. Phys. 49879 (1987)

[105] P.G.J. van Dongen and M.H. Ernst, "Fluctuations in coagulating systems II", J. Stat. Phys. 49927 (1987)

[106] P.G.J. van Dongen and M.H. Ernst, "Solutions of Smoluchowski's Coagulation Equation at Large Cluster Sizes", Physica A 14515 (1987)

[107] P.G.J van Dongen, "On the possible occurrence of instantaneous gelation in Smoluchowskis coagulation equation", J. Phys. A: Math. Gen. 201889 (1987)

[108] P.G.J. van Dongen and M.H. Ernst, "Scaling solutions of Smoluchowski's coagulation equation", J. Stat. Phys. 50295 (1988)

[109] P.G.J. van Dongen, "Upper critical dimension in irreversible aggregation". Phys. Rev. Lett. 631281 (1989)

[110] T. Vicsek and F. Family, "Dynamic scaling for aggregation of clusters", Phys. Rev. Lett. 521669 (1984)

[111] T. Vicsek and P. Meakin, "Scaling in steady-state cluster-cluster aggregation", Phys. Rev. A 321122 (1985)

[112] W.H. White, "A global existence theorem for Smoluchowski's coagulation equations", Proc. Am. Math. Soc. 80273 (1980) 
[113] W.H. White, "On the form of steady-state solutions to the coagulation equations", J. Colloid and Interface Sci. 87204 (1982)

[114] D. Williams, "Probability with Martingales", Cambridge University Press (1991)

[115] R.M. Ziff and G. Stell, "Kinetics of polymer gelation", J. Chem. Phys. 733492 (1980)

[116] R.M. Ziff, E.M. Hendriks and M.H. Ernst, "Critical properties for gelation: A kinetic approach", Phys. Rev. Lett. 49593 (1982)

[117] R.M. Ziff, M.H. Ernst and E.M. Hendriks, "Kinetics of gelation and universality", J. Phys. A 162293 (1983)

[118] R.M. Ziff, M.H. Ernst and E.M. Hendriks, "A Transformation Linking Two Models of Coagulation", J. Colloid and Interface Sci. 100220 (1983) 